Portland State University

PDXScholar

Spring 6-9-2016

\title{
A Utility-Scale Deployment Project of Behind-the- Meter Energy Storage for Use in Ancillary Services, Energy Resiliency, Grid Infrastructure Investment Deferment, and Demand-Response Integration
}

Joseph Nathanael Wilson

Portland State University

Follow this and additional works at: https://pdxscholar.library.pdx.edu/open_access_etds

Part of the Power and Energy Commons

Let us know how access to this document benefits you.

\section{Recommended Citation}

Wilson, Joseph Nathanael, "A Utility-Scale Deployment Project of Behind-the-Meter Energy Storage for Use in Ancillary Services, Energy Resiliency, Grid Infrastructure Investment Deferment, and Demand-Response Integration" (2016). Dissertations and Theses. Paper 3004.

https://doi.org/10.15760/etd.3005

This Thesis is brought to you for free and open access. It has been accepted for inclusion in Dissertations and Theses by an authorized administrator of PDXScholar. Please contact us if we can make this document more accessible: pdxscholar@pdx.edu. 
A Utility-Scale Deployment Project of Behind-the-Meter Energy Storage for Use in Ancillary Services, Energy Resiliency, Grid Infrastructure Investment Deferment, and Demand-Response Integration

by

Joseph Nathanael Wilson

A thesis submitted in partial fulfillment of the requirements for the degree of

Master of Science

in

Electrical and Computer Engineering

Thesis Committee:

Robert Bass, Chair

James McNames

Linda Rankin

Shawn Chandler

Portland State University

2016 
(C) 2016 Joseph Nathanael Wilson 


\section{Abstract}

Electric utilities are increasingly incentivized to integrate new renewable energy generation resources to their systems; however, operations-related issues arise due to the non-dispatchable and stochastic nature of these renewable energy sources. These characteristics lead to a variety of issues for utilities, among which are voltage fluctuations, balancing dispatch against ramping events, short-duration power fluctuations, and the need to invest in peaking generation facilities just to accommodate the renewable energy.

A traditional solution to these issues is to employ renewable generation-following techniques using either newly constructed gas peaking plants, or by shifting existing generation resources to this following responsibility. Unfortunately, use of these traditional methods introduces a new set of issues; namely, wear-and-tear due to more frequent cycling, reduced capacity factors, decreased plant efficiency, and additional investment in large-scale captital infrastructure.

This thesis proposes an alternate solution: a utility-owned and utility-managed battery energy storage system sited on residential customer premises, deployed at scale to create a 200MW / 1320MWh distributed network of Residential Battery Energy Storage Systems (ResBESS). In partnership with Portland General Electric 
(PGE) stakeholders, a conceptual design was prepared for a ResBESS unit, a laboratory prototype of a single such storage system was constructed, and an alpha prototype is now being installed in a field demonstration project in Milwaukie, Oregon within PGE's service territory. The motivations, design constraints, and design methodology of the laboratory prototype are presented and discussed, and preliminary work from the field prototype build is examined to demonstrate the results of the thesis project. 


\section{Contents}

Abstract

List of Tables $\quad$ vi

List of Figures $\quad$ vii

1 Introduction 1

1.1 Problem Statement . . . . . . . . . . . . . . . . 1

1.1.1 Details about Problem Statement ........... 2

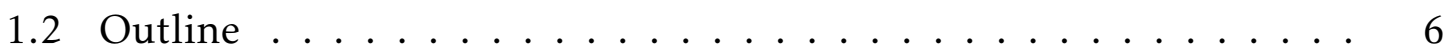

2 Objective \& Benefits 8

2.1 Project Objective . . . . . . . . . . . . . . . . 10

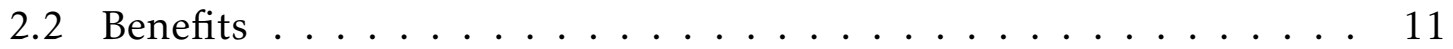

2.2.1 Utility Benefits .................. 11

2.2.2 Customer Benefits . . . . . . . . . . . . . 13

3 Literature Summary 17

4 General Design Considerations and Constraints 24

4.1 Future Meter Description . . . . . . . . . . . . . . . . . 24

4.1 .1240 Volt Connection . . . . . . . . . . . . 25

4.1.2 Ports, Points and Nodes . . . . . . . . . . . 26

4.1 .3 Disconnect Switches . . . . . . . . . . . . . . . 27

4.1.4 Communications and Control ..................... 28

4.1 .5 Neutral Connection . . . . . . . . . . . . . . . 34

4.2 Desired System States and Transitions . . . . . . . . . . . . 35

4.2.1 System State Definitions . . . . . . . . . . . . . . . 35

4.2.2 Methods of Switching . . . . . . . . . . . . 38

4.2.3 State Transition Switching Logic . . . . . . . . . . . . 39

4.2.4 Utility Control and Data Handling . . . . . . . . . . . 41

4.3 Use Cases . . . . . . . . . . . . . . . . . . . . 47

4.3.1 Use-Cases Defined . . . . . . . . . . . . . . . . . 47

4.4 Use-Case Motivations . . . . . . . . . . . . . . . . . . . 53

4.4.1 Use-Case Technical Needs . . . . . . . . . . . . . 54 
4.5 Technical Specifications . . . . . . . . . . . . . . . 56

5 Prototype Design Considerations and Constraints 58

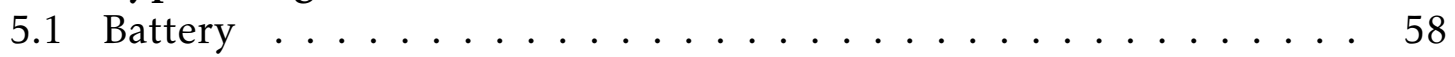

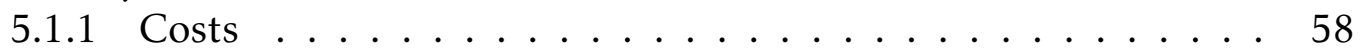

5.1 .2 Aquion Battery . . . . . . . . . . . . . . . . . . . 59

5.1 .3 Batteries Used in Prototype . . . . . . . . . . . . . . . . 62

5.2 Inverter . . . . . . . . . . . . . . . 62

5.2 .1 Inverter Choice $\ldots \ldots \ldots \ldots$

5.2 .2 Inverter Constraints . . . . . . . . . . . . . . . 64

5.2.3 Constraints Involving Aquion Battery . . . . . . . . . . . . 65

5.3 Meter . . . . . . . . . . . . . . . . 67

5.3.1 Simulating Meter Communications and Control . . . . . . . 68

5.3.2 Linking the RTAC to Prototype Devices . . . . . . . . . . . 69

5.3.3 Data Provided to PGE using RTAC to Prototype Device Connections . . . . . . . . . . . . . . . 73

6 Conclusion \& Outcomes $\quad 75$

6.1 Future Work Underway . . . . . . . . . . . . . . . . 76

6.1 .1 Service Access . . . . . . . . . . . . . . . . . . . . 79

6.1.2 Inverter Constraints Addressed . . . . . . . . . . . . 81

6.1 .3 RTAC Control Loop . . . . . . . . . . . . . . . . . . . 83

$\begin{array}{ll}\text { Bibliography } & 86\end{array}$

$\begin{array}{ll}\text { Appendices } & 92\end{array}$

Appendix A: Supplementary Analysis $\quad 92$

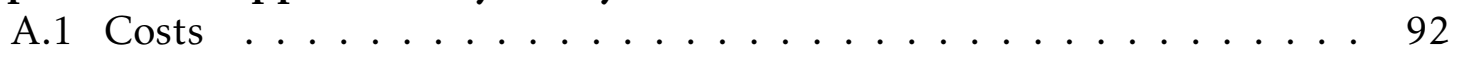

A.2 Maintenance ..................... . . 95

$\begin{array}{ll}\text { Appendix B: Summary Table } & 97\end{array}$

Appendix C: Technical Specifications $\quad 99$

C.1 Meter Specifications . . . . . . . . . . . . . . . . . . . . 99

C.2 Inverter Specifications . . . . . . . . . . . . . . . 102

$\begin{array}{ll}\text { Appendix D: IEEE } 1547 & 106\end{array}$

$\begin{array}{ll}\text { Appendix E: NERC CIP } & 108\end{array}$

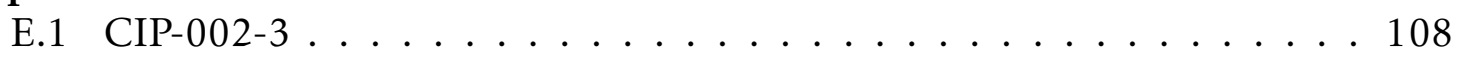

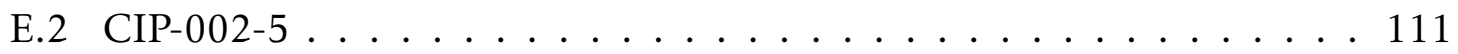

E.2.1 ResBESS and CIP-002 . . . . . . . . . . . . . . . 115 
Appendix F: Additional Device Drawings and System Schematics

F.1 Meter-Base Adapter . . . . . . . . . . . . . . . . . . . 116

F.2 Field Prototype . . . . . . . . . . . . . . . 116

Appendix G: Switching Logic 123

G.1 Grid Tied State Transitions . . . . . . . . . . . . . . . . . . . . . . 123

G.2 Maintenance State Transitions . . . . . . . . . . . . . . . . 126

G.3 Backup State Transitions . . . . . . . . . . . . . . . . . . . . . . 129

G.4 Service Disconnect State Transitions . . . . . . . . . . . . . . . 132

G.5 No Service State Transitions . . . . . . . . . . . . . . . . . . . 135

Appendix H: RTAC Logic $\quad 140$

Appendix I: Equations 143

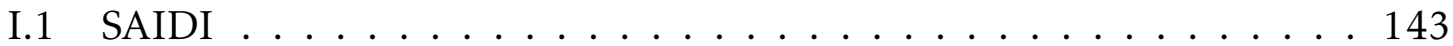

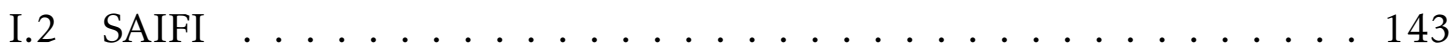

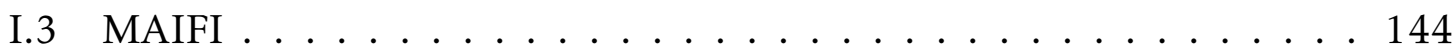


List of Tables

2.1 PGE Outage Numbers Presented to OPUC, February 2015 . . . . . . . 14

2.2 Estimated Customer Interruption Costs - Residential . . . . . . . . . . 15

4.1 Truth table for the Status Signals and the resulting Switch Positions and Systems States. . . . . . . . . . . . . . . . . . . 41

5.1 PGE to PSU Register List, Dec '15 . . . . . . . . . . . . . . . 73 


\section{List of Figures}

1.1 United States and Territories Renewable Portfolio Standards as of Octo-

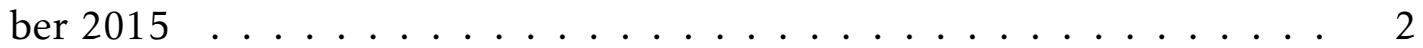

1.2 Scheduled Electric Generation Capacity Additions for 2016 . . . . . . 4

2.1 Conceptual Drawing of Single ResBESS Unit, Showing Primary Components ......................... 9

3.113 Possible Services for Battery Energy Storage . . . . . . . . . . . 20

4.2 The state transition diagram for four ResBESS basic use cases. Transitioning between one state and another requires opening and/or closing combinations of the three disconnect switches. . . . . . . . . . 37

4.3 System Operator view showing the overview of the system. . . . . . . 42

4.4 System Operator view showing the details of an individual unit. . . . . 44

4.5 System Operator view showing the health of the system. . . . . . . . . . 45

4.1 The basic functional blocks, metering points and disconnect switches within the housing of the ResBESS meter. . . . . . . . . . . . 57

5.1 Battery performance data at continuous current: red lines show inverter current limits and orange lines show inverter voltage limits. . . . . . . 66

6.1 "One-line" Drawing of ResBESS Field Prototype Primary Components . 78

6.2 Split-Phase Wiring Diagram for Breaking Customer Service . . . . . . . 80

6.3 Desired-Power Discharge Step-Response Control-Loop Test Data . . . . 84

6.4 Load-Matching (Peak Shaving) Control-Loop Test Data . . . . . . . . . . 85 


\section{Introduction}

\subsection{Problem Statement}

Electric utilities are increasingly motivated to increase the penetration of renewables on the power grid; however, operations-related issues have arisen in prominence due to the non-dispatchable and stochastic nature of these renewable energy sources. These characteristics lead to a variety of issues for utilities, among which are voltage fluctuations, balancing dispatch against ramping events, short-duration power fluctuations, and the need to invest in distant peaking generation facilities just to accommodate renewables. [1][2]

Secondarily, as costs drop for both rooftop solar arrays (and their associated balance-of-system components) and for various battery options for home use, utilities are faced with the possibility of either "grid defection" as customers invest

in their own energy resources, or increased competition as $3^{\text {rd }}$-party aggregators bundle together many homes with solar and/or storage and engage in wholesale markets. 


\subsubsection{Details about Problem Statement}

Despite the problems that renewable energy may cause, there are a variety of reasons for a utility to be interested in making investments in renewable generation resources, including renewable portfolio standards (RPS) mandated by state regulators - see Figure $1.1^{1}$, pressure from their customers and society at large, and the increasing cost-effectiveness of such investments.

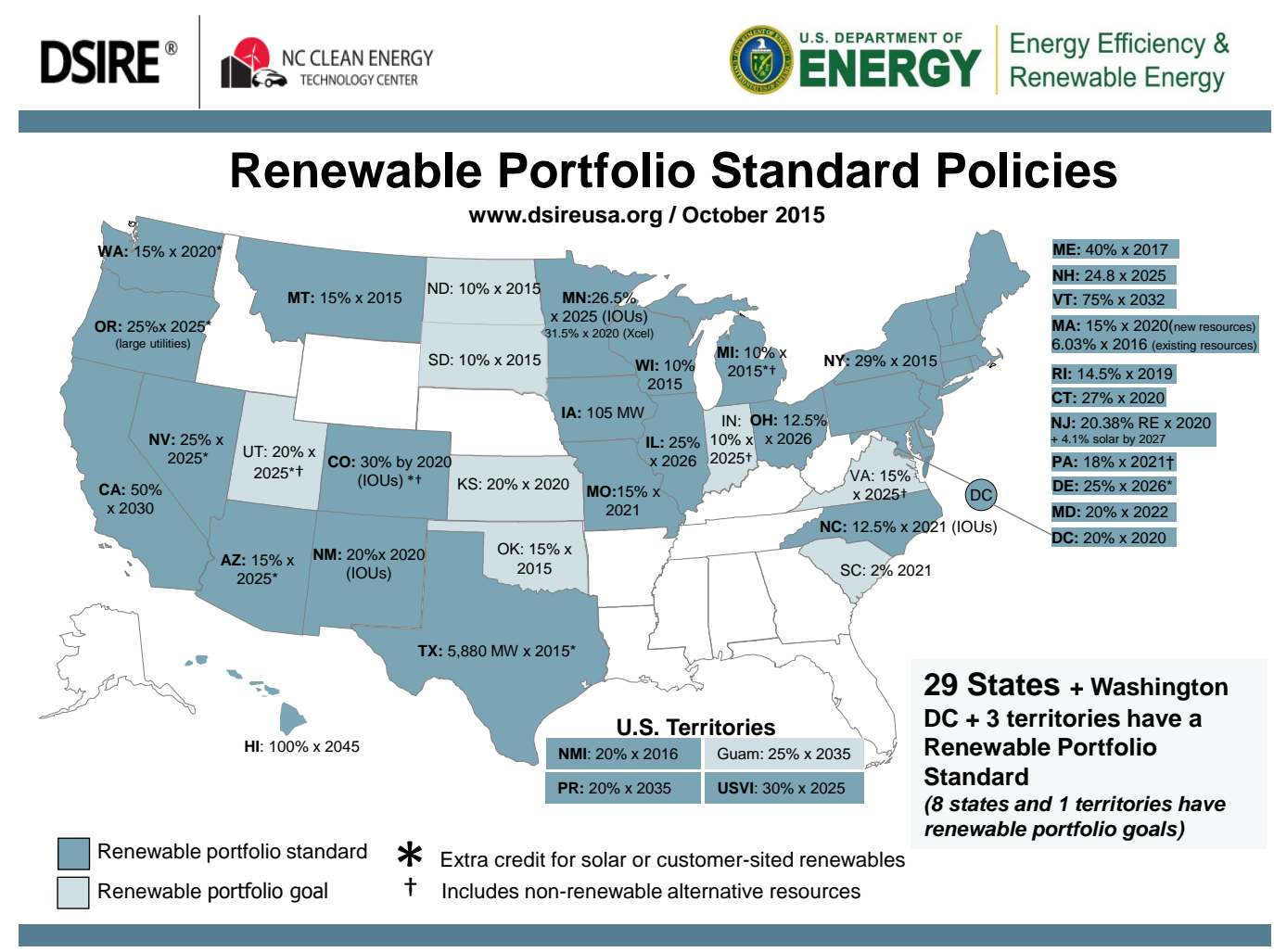

Figure 1.1: United States and Territories Renewable Portfolio Standards as of October 2015 Source: Database of State Incentives for Renewables \& Efficiency [3]

These drivers of renewables, further pushed by the extension until 2020 of the federal renewable electricity production and energy investment tax credits, are

\footnotetext{
${ }^{1}$ Oregon's RPS was just revised to $50 \%$ by 2040
} 
evidenced by the large share of new renewable generation sources scheduled for installation in 2016 (see Figure 1.2), and thus the operational issues mentioned previously are growing and require immediate attention from utilities. A traditional solution to these issues is to employ renewable generation-following techniques detailed in Subsection 4.3.1.4 - using either newly constructed gas peaking plants, or by shifting existing generation resources to this following responsibility. For example, Portland General Electric (PGE) testified to the Oregon Public Utility Commission that it was for this very reason that Port Westward II (a 224 MW gasfired reciprocal engine set) was constructed.[4] However, use of these traditional methods can introduce a new set of issues; namely, wear-and-tear due to more frequent cycling, reduced capacity factors, and decreased plant efficiency. In fact, in a report from the National Renewable Energy Laboratory (NREL), entitled The Western Wind and Solar Integration Study Phase 2, it was found that at a 33\% penetration of wind and solar in the Western Interconnect, there would be cycling costs of $\$ 35$ million to $\$ 157$ million incurred per year, with the conclusion that "[f]or the average fossil-fueled plant, this results in an increase in operations and maintenance costs of $\$ 0.00047$ to $\$ 0.00128$ per kilowatt-hour of generation." [5] A traditional solution thus involves either reducing the capacity factor and/or efficiency of existing generation resources, or spending the money to build new highly-flexible generation assets in addition to an investment made in a renewable generation resource. 


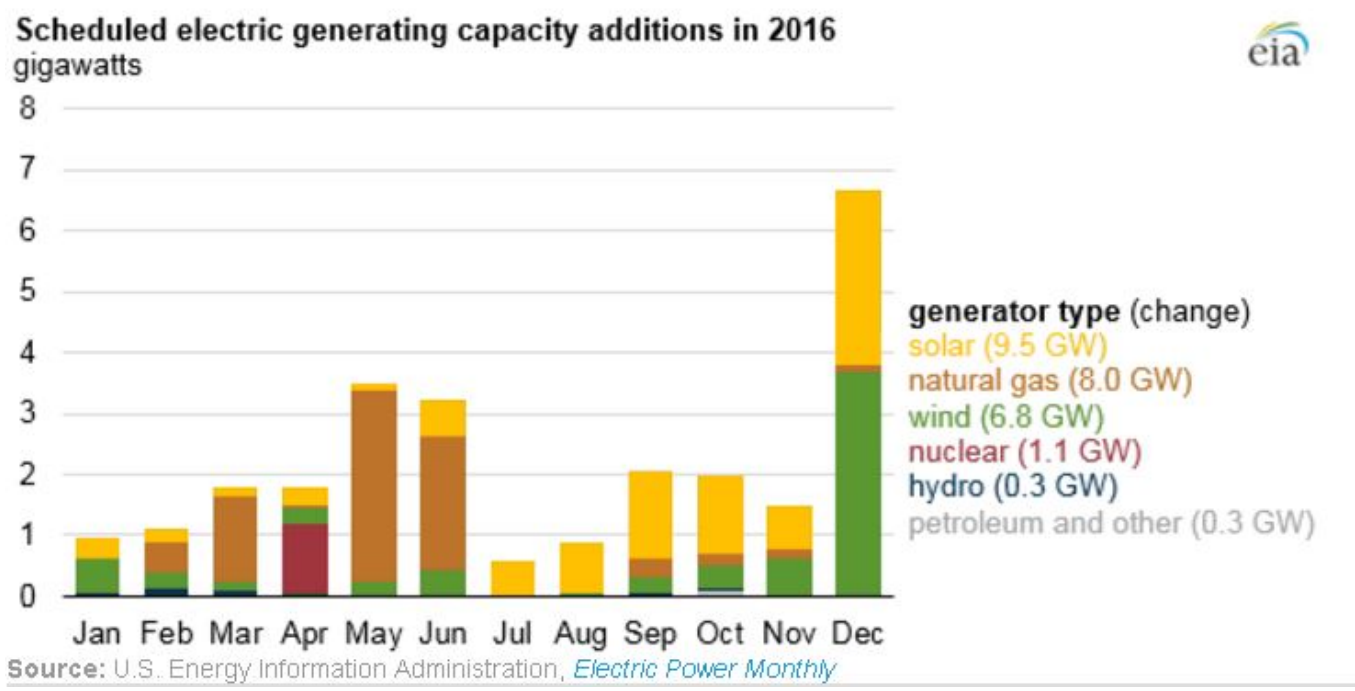

Figure 1.2: Scheduled Electric Generation Capacity Additions for 2016 Source: U.S. Energy Information Administration [6]

Finally, as battery energy storage options drop in price, the possibility arises for non-utility-owned and operated battery systems to be installed at utility customer premises. While not a strong driver of the proposed project, there is a future possibility that distributed energy resource aggregators (or individual customers) could operate these storage resources as they see fit; charging and discharging them as desired, possibly driven solely by market forces and without regard for the health of the power grid. If the batteries are installed with a rooftop solar array, there is even the possibility for some customers to eliminate their need for a utility connection. Because of the way that utility rates are usually structured, for each customer who pairs solar PV with storage, any utility revenue which has been rate-based - revenue that may have been budgeted for grid operations and maintenance - could be removed from the utility's cash-flow. This can result in a 
detrimental feedback loop: by cutting this revenue stream, there is the long-term possibility of decreasing the ability of the utility to make grid investments that the public may desire, which can further drive the desire of customers to defect from the utility, which can reduce the utility's ability to invest in the reliability of the grid, etc.

A way to support the desire of utilities to add renewable generation resources to their portfolio, while simultaneously introducing a possible solution to many or most of these problems, is a large network of utility-owned and controlled Residential Battery Energy Storage Systems (ResBESS). In addition to helping a utility address the operational issues previously listed - voltage fluctuations, ramping events, short-duration power fluctuations, etc. - such a network could also help to address traditional operational challenges such as meeting peak demand and providing economic arbitrage. Rather than relying on generating assets, or using large, centralized battery banks (which might not be very close to load centers, and would thus incur line losses while charging and again while discharging), a network of tens of thousands of ResBESS dispersed throughout a balancing area could be used in aggregate to provide services that alleviate these challenges. Locating these units at or near residences would reduce distribution line losses upon discharge - compared to distantly-sited storage resources, and could reduce all traditional load-driven line losses if they can be charged off-peak: shifting load away from peak times would reduce line congestion on-peak, and thus could reduce $I^{2} R$ losses 
at these heavy-load times.

Research into this possible solution has been undertaken by the Power Engineering Group at Portland State University, in partnership with PGE. This thesis presents the results of that research: engineering knowledge intended to support the construction and installation of a single ResBESS field prototype unit by future researchers. This knowledge is already being applied towards the design and construction of a field prototype by a new team of researchers, and their work is included as appropriate.

\subsection{Outline}

Due to the amount of content and information compiled, this document is divided up into several sections. For ease of reading, a brief outline is provided here.

First, the objectives of the project are outlined, along with a high-level examination of the benefits a system of ResBESS would provide to both utilities and customers. This is followed immediately by Section 3 and a short literature summary of similar solutions proposed by others.

After this, in Section 4 we review the design considerations for a conceptual ResBESS and a system of such units, including future components needed and methods of communication and control. These conceptual constraints are then realized in the following section, where the components used in the laboratory prototype are studied and detailed, along with limitations imposed by these real- 
world devices.

Finally, the conceptual and actual are combined in Section 6, where a review of the previous sections is undertaken, along with a short discussion of how the knowledge of the thesis project is being used by a current research team.

Some additional miscellaneous content is included in Section A - namely, highlevel cost and failure-mode analyses - as well as in the many appendices which are referenced throughout this document. 


\section{Objective \& Benefits}

Over the past two-and-a-half years, the Power Engineering Group at Portland State University has been researching the deployment of a distributed network of Residential Battery Energy Storage Systems. A high-level conceptual drawing of a single ResBESS unit is shown in Figure 2.1. A single ResBESS unit would consist of a battery sited on customer premises, which would then be tied into both the home and the grid through a bi-directional inverter and smart-meter (both these devices being designed specifically for this use). At least one switch would be desired to allow the ResBESS unit to use its stored energy for discharge to the residence in the case of an outage. 


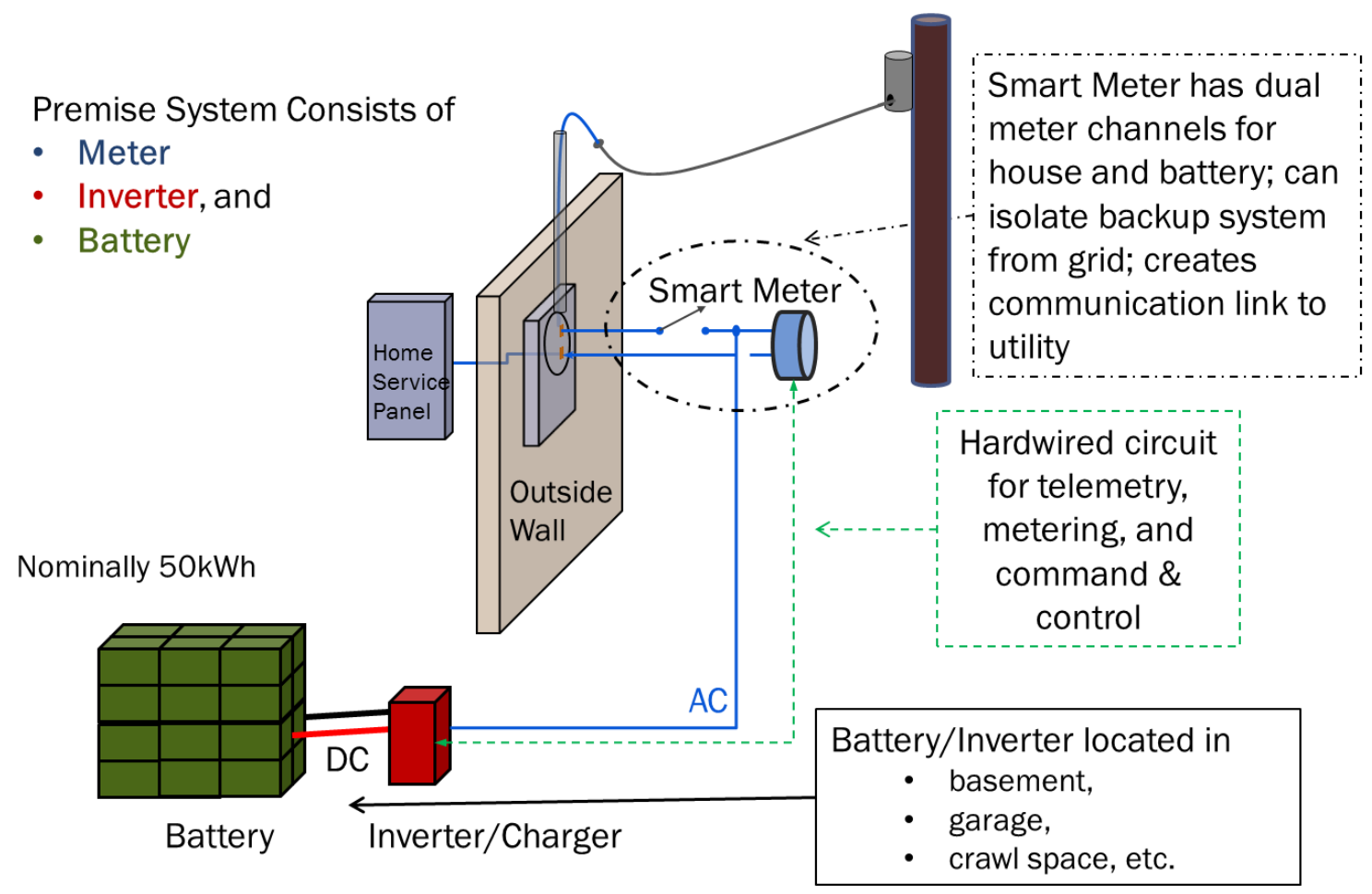

Figure 2.1: Conceptual Drawing of Single ResBESS Unit, Showing Primary Components

In this deployment, a network of tens of thousands of ResBESS units, stored within customer homes and commercial buildings, would be used in aggregate to provide a number of services. A selection of these services are briefly listed here, with more details provided in section 4.3.1. The services that aggregated storage could provide include firming services to buffer load and generation ramping events, countering power fluctuations presented by distributed renewable resources, resiliency for the customer in the event of power system outages, maintaining revenue to the utility while taking the home off-grid during peak load times, discharging or charging as needed to regulate frequency, and shaping services by supplying energy during peak demand periods and storing it during periods of 
low demand. Additionally, using a four-quadrant inverter, localized VAr support could be layered on top of any these other real power services. Finally, when used for arbitrage, these batteries could allow a utility to avoid the need to make unscheduled purchases of energy from the spot market.

\subsection{Project Objective}

The objective of the thesis project was to gain the engineering knowledge necessary to support the construction and installation of a single ResBESS field prototype unit by future researchers, while keeping in mind that the end-goal is a large system of ResBESS units, as explained previously. To obtain this body of knowledge, a decision was made to construct - using off-the-shelf components wherever possible - a battery and inverter system laboratory prototype that could charge and discharge upon command via signals from the utility, as well as demonstrate the ability to provide a customer with backup power in the case of an outage. Concurrently, an understanding of the long-term goals of the project was to be fostered, including a list of specifications for prototype systems to be developed in future. These specifications ideally would be further developed by future researchers in cooperation with manufacturing partners; to this end, relationships were built with Aquion Energy, Outback Power, and Marwell Corporation for battery, inverter, and meter-base-internals access respectively. Preliminary discussions with Elster Group about a specialized meter have also occurred, but no real partnership has been 
formed as of yet.

\subsection{Benefits}

There is currently no program of utility-owned energy storage sited at customer premises at the scale proposed within this project. However, were such a network of ResBESS units to be implemented, the costs and benefits for customers and the utility would be shared, though not equally. The utility would finance the majority of the capital, installation and operational cost while the customer would pay only a small monthly service fee. This imbalance reflects the fact that the utility would receive a majority of the benefits, being able to use the units to manage dispatch and provide economic arbitrage; whereas unless the utility chooses to extend other incentives to them, the customer would only benefit through an increase in reliability against outages. Nonethless, this is a unique opportunity for a regulated, investor-owned utility to partner with its customers in a way that benefits both parties.

\subsubsection{Utility Benefits}

Compelling reasons exist for a utility to invest in a network of ResBESS. As previously mentioned, in aggregate, such a network would help address load and generation ramping events, voltage fluctuations, peak demand, frequency events, and engage in economic arbitrage. At $5 \%$ market adoption $(\approx 25,000$ units), PGE could control an approximately 200 MW, 1,320 MWh storage resource. From its 
operations center, PGE would be able to control these distributed units as a single aggregated resource, or as a strategically-segmented group of resources.

The battery systems would be available for economic arbitrage, in addition to power-related functions such as meeting peak demand, firming and shaping in support of variations from renewables, alleviating voltage fluctuations and providing increased reliability for a significant group of utility customers.

Financially, this adoption rate of $5 \%$ would provide a multi-million dollar investment opportunity for the utility. At the price-per-unit outlined in this thesis (see section A.1), a system of 25,000 ResBESS units aggregated to provide 200MW and 1320 MWh of capacity would be comparable to a newly-constructed natural gas peaking plant; e.g. PGE's Port Westward II plant (nameplate $224 \mathrm{MW}$ ) had capital costs of approximately $\$ 300 \mathrm{~mm}$, while this project proposes capital costs of $\$ 364 \mathrm{~mm}$ - more than Port Westward II, but with added value provided to the utility by adding a demand-response and storage resource. The Port Westward project also has $\$ 1.4 \mathrm{~mm}$ budgeted annually for property taxes - costs which can be completely avoided for the proposed system of ResBESS units due to the units being sited on customer property. [4]. A comparison has not been made between estimated operating and maintainance (O\&M) costs for a network of ResBESS and the budgeted O\&M costs for Port Westward II - future researchers may desire to undertake this analysis. 


\subsubsection{Customer Benefits}

Because of its location behind the meter, ResBESS could provide a participating customer with backup power during outages, which, while infrequent, could otherwise negatively impact those with in-home medical equipment ${ }^{2}$ or home businesses. This backup power would be available to the customer for a fraction of what a backup system would otherwise cost while also being maintenance-free. Future researchers will need to consider the length of time which this backup power could be promised to cover, as well as understand the utility's responsibility to pre-charge the battery to provide this customer service.

Due to the proposed use of the ResBESS as a back-up power source for the customer whose property houses the unit, the costs of outage are reduced for both the customer and utility.

There are three metrics for measuring outages:

- System Average Interruption Duration Index (SAIDI)

- Average outage duration for a customer in a year

- Defined in Equation I.1

- System Average Interruption Frequency Index (SAIFI)

- Average number of interruptions that a customer would experience in a year

\footnotetext{
${ }^{2}$ Many critical medical devices already have their own battery backup; this may not be a strong selling point for ResBESS
} 
- Defined in Equation I.2

- Momentary Average Interruption Frequency Index (MAIFI)

- Average number of momentary interruptions ${ }^{3}$ that a customer would experience in a year

- Defined in Equation I.3

According to PGE testimony given to the Oregon Public Utility Commission (OPUC) in February 2015 (data shown in Table 2.1), the average time of outage for their customers (from 2012-2014) is 76 minutes, while a single customer is expected to have 0.6 outages in year, and 1.1 momentary outages in a year. [7]

Table 3

Three-year Weighted Averages and Penalty Threshold Limits

Year

2014

2013

2012

\section{3-Year Weighted Average}

OPUC Level 1 Penalty

Threshold
SAIDI

(minutes)

93

62

72

76

105
SAIFI (occurrences)

0.7

0.5

0.6

0.6

1.2
MAIFI (occurrences)

1.3

0.9

1.1

1.1

5.0

Table 2.1: PGE Outage Numbers Presented to OPUC, February 2015 [7]

The data seen in Table 2.2, taken from the Berkley National Laboratory's most recent Value of Service Reliability Estimates for Electric Utility Customers in the United States report, help to quantify these PGE reliability numbers in financial terms [8].

\footnotetext{
${ }^{3}$ PGE uses 5 minutes or less to define "momentary."
} 
Estimated Customer Interruption Costs (U.S.2013\$) by Duration and Timing of Interruption - Residential

\begin{tabular}{|c|c|c|c|c|c|c|c|}
\hline \multirow{2}{*}{ Timing of Interruption } & \multirow{2}{*}{$\begin{array}{c}\% \text { of } \\
\text { Hours } \\
\text { per Year }\end{array}$} & \multicolumn{6}{|c|}{ Interruption Duration } \\
\hline & & Momentary & 30 Minutes & 1 Hour & 4 Hours & 8 Hours & 16 Hours \\
\hline Summer Morning/Night & $19 \%$ & $\$ 6.8$ & $\$ 7.5$ & $\$ 8.4$ & $\$ 14.3$ & $\$ 24.0$ & $\$ 42.4$ \\
\hline Summer Afternoon & $7 \%$ & $\$ 4.3$ & $\$ 4.9$ & $\$ 5.5$ & $\$ 9.8$ & $\$ 17.1$ & $\$ 31.1$ \\
\hline Summer Evening & $7 \%$ & $\$ 3.5$ & $\$ 4.0$ & $\$ 4.6$ & $\$ 9.2$ & $\$ 17.5$ & $\$ 34.1$ \\
\hline Non-summer Morning/Night & $39 \%$ & $\$ 3.9$ & $\$ 4.5$ & $\$ 5.1$ & $\$ 9.8$ & $\$ 17.8$ & $\$ 33.5$ \\
\hline Non-summer Afternoon & $14 \%$ & $\$ 2.3$ & $\$ 2.7$ & $\$ 3.1$ & $\$ 6.2$ & $\$ 12.1$ & $\$ 23.7$ \\
\hline Non-summer Evening & $14 \%$ & $\$ 1.5$ & $\$ 1.8$ & $\$ 2.2$ & $\$ 5.0$ & $\$ 10.8$ & $\$ 23.6$ \\
\hline \multicolumn{2}{|l|}{ Weighted Average } & $\$ 3.9$ & $\$ 4.5$ & $\$ 5.1$ & $\$ 9.5$ & $\$ 17.2$ & $\$ 32.4$ \\
\hline
\end{tabular}

Table 2.2: Estimated Customer Interruption Costs - Residential [8]

Using PGE's average SAIDI number of 76 minutes per outage, we choose the 1 hour interruption values for analysis, and can see a range of $\$ 2.20-\$ 8.40$ in costs per average outage for each residential customer, with a weighed average cost of $\$ 5.10$ per outage. There is nothing that precludes the ResBESS unit from being sized to be placed at a small industrial or commercial site; interruption costs are even higher for these customers. If the system is engineered well, then the SAIDI, SAIFI,\& MAIFI numbers would drop to near zero for every customer with a ResBESS unit - good for PGE, since these numbers are tracked by regulating agencies for compliance; and the customer would save money and hassle related to outages - good for the customer \& PGE's relationship with the customer, since the customer would perceive greater reliability from the utility.

Finally, customers would also gain a sense of value, knowing they are facilitating the deployment of clean energy resources and the implementation of novel "smart grid" technologies. The proposed system would allow customers to assist with 
the deployment of more renewable resources with little effort required on their part, which could prove to be a bonus for PGE, as many of their customers are keen to participate in utility programs that benefit the environment; for instance, PGE currently has around 15\% of its customers enrolled in voluntary clean energy programs where they pay a premium every month to support renewable energy. ${ }^{4}$ It remains to be seen if this will translate into customers who are willing to house an energy storage resource on their property. Future work must be done to gauge the market demand for a product like ResBESS.

\footnotetext{
${ }^{4}$ This is 125,000 customers; $500 \%$ of the number desired to fully scale the ResBESS system
} 


\section{Literature Summary}

Within literature, there are no solutions that have been published in conference proceedings or journal articles that are similar to the ResBESS systems. There are many papers that model a theoretical residential storage system, but in which no physical BESS is constructed or operated. For instance, in Autonomous demand-side optimization with load uncertainty, a team modeled several residences with nonadjustable loads, adjustable loads and a storage device, all of which utilize an energy consumption scheduler to run the adjustable loads and charge/discharge of the storage device. [9] R. S. Weissbach et al. have published several papers regarding use of energy storage in an off-grid residence (the ResBESS is grid-tied). Their work is largely statistical modeling - in one paper they use iterative methods and a Markov model to determine the amount of energy storage required for reliable power in the theoretical residence; while in another paper, they employed Monte Carlo simulations to determine the number of hours where load could not be supplied by a combination of wind and battery storage. Once again, these papers involve only modeling methods and results, with no physical system in place. [10][11]

There are also many articles that propose systems at a larger scale, such as work by Krishnamoorthy et al., where a theoretical $1100 \mathrm{~V}, 2 \mathrm{MW}, 20 \mathrm{~Hz}_{\max }$ wind turbine 
is tied to $900 \mathrm{Vdc} 1 \mathrm{MW} / 3 \mathrm{MWh}$ lithium-ion battery energy storage through a 3port topology - they propose use of a $0.2-4 \mathrm{kHz}$ square wave to modulate the $20 \mathrm{~Hz}$ output of the turbine into a medium-frequency transformer and inverter system for use in a 50 or $60 \mathrm{~Hz}$ grid - and offer a control system able to handle voltage sags/swells and provide low voltage ride through (LVRT) capability. [12] Again, theoretical work - no equipment is specified that we could use for comparison to the components selected for the prototype discussed in this thesis. A utility scale battery energy storage system for intermittency mitigation in multilevel medium voltage photovoltaic system; and Enhanced Security-Constrained Unit Commitment With Emerging Utility-Scale Energy Storage [12][13][14].

The missing piece in these academic papers and journal articles, is details about the physical components that would need to be assembled to achieve the goals and models that the authors are proposing. As I have learned over the course of this project, there is a great difference between saying theoretically that an inverter is needed, and working with a specific brand of inverter that has unique characteristics and constraints.

Bridging the gap between theoretical academic work and the practical knowledge we desire is a paper published in the peer-reviewed journal Nature Climate Change, where Nykvist and Nilsson bring together over 80 different estimates of capital cost data from both academic and non-academic sources that were reported between 2007-2014, to develop an understanding of recent cost trends for batter- 
ies. [15] These data are specific to Li-ion electric vehicle (EV) battery packs, rather than utility BESS; however, the EV market is a significant driver of battery costs and Li-ion technologies are often used for utility BESS - Tesla's new utility-scale Powerpack is a most recent example. The authors found that while cost estimates for these batteries declined around 14\% annually between 2007 and $2014(\$ 1,000 / \mathrm{kWh}$ to $\$ 410 / \mathrm{kWh}$ ), the actual cost of battery packs that electric vehicle manufacturers used was even lower, at $\$ 300$ per $\mathrm{kWh}$. Their analysis of the available data also predicts that Li-ion capital costs will continue to decline, settling asymptotically within the $\$ 150-\$ 300 / \mathrm{kWh}$ price range by 2025 .

Additionally, a recent report from Navigant entitled Community, Residential, and Commercial Energy Storage predicts that global revenue from distributed BESS is expected to grow from $\$ 452$ million annually in 2014 to more than $\$ 16.5$ billion in 2024, and globally installed distributed BESS power capacity is expected to grow from 171.9 MW in 2014 to $12,147.3 \mathrm{MW}$ in 2024. [16].

With costs of batteries predicted to drop, and the market for distributed energy stoage predicted to grow, we seek to now justify our claims for the purported value storage can provide to the grid and electric utilities. The Rocky Mountain Institute (RMI) published The Economics of Battery Energy Storage: How multi-use, customer-sited batteries deliver the most services and value to customers and the grid in September 2015. [17] In their report, they identify 13 areas where energy storage can provide value, separated into services for customers, utilities, or independent 
system operators/regional transmission organizations (ISO/RTOs) - an illustration of these services is shown in Figure 3.1. Interestingly, behind-the-meter storage, as proposed in this thesis, can meet all 13 of their identified value areas; while storage installed on the transmission and distribution system cannot.

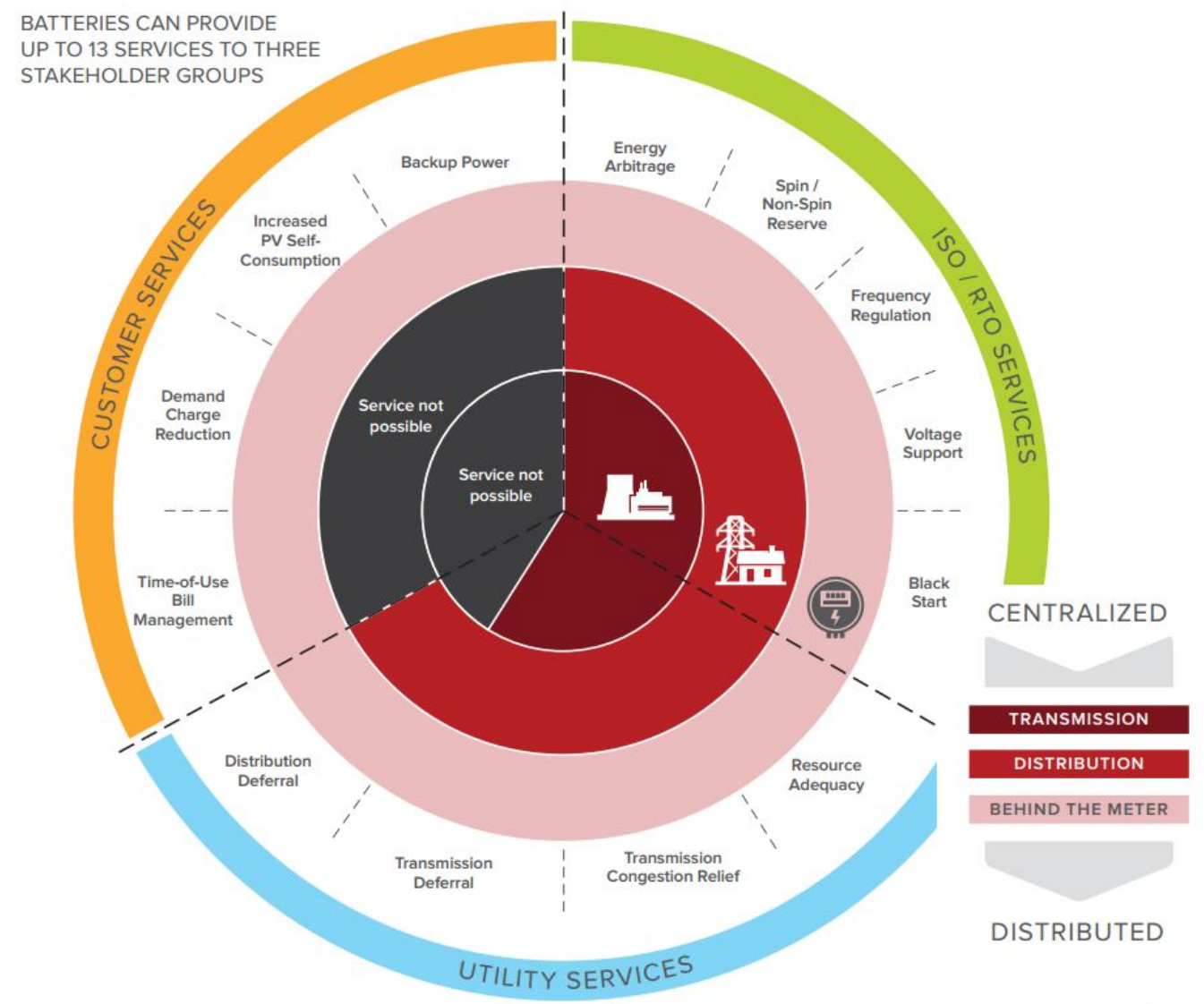

Figure 3.1: 13 Possible Services for Battery Energy Storage Source: Rocky Mountain Institute [17]

RMI examines four use cases for energy storage: commercial demand-charge management in San Francisco, distribution upgrade deferral in New York, residential bill management in Phoenix, and solar self-consumption in San Francisco. All of these use cases were found to provide value, but those that could contribute 
to deferral of distribution or transmission upgrades had the largest $\$ / \mathrm{kW}$ value. Finally, and most importantly, the Institute found that most currently installed distributed storage systems are deployed for only a single application, leaving a significant amount of value un-realized. To wit, they suggest that " $[u]$ nder prevailing cost structures, batteries deployed for only a single primary service generally do not provide a net economic benefit (i.e., the present value of lifetime revenue does not exceed the present value of lifetime costs), except in certain markets under certain use cases", which would seem to put an end to the justification for this project. They continue on however, finding that "...given that the delivery of primary services only takes $1-50 \%$ of a battery's lifetime capacity, using the remainder of the capacity to deliver a stack of services to customers and the grid shifts the economics in favor of storage." This suggests that future researchers should endeavour to build on this project with layering of storage services as a primary goal.

We now turn to some examples of current utility-run energy storage demonstration projects. For example, Austin Energy in Austin, Texas is using a a $\$ 4.3$ million grant from the Department of Energy's new SHINES (Sustainable and Holistic Integration of Energy Storage and Solar PV) program to demonstrate a system of solar PV, battery storage, thermal storage, and demand response. They have announced partnerships with Tesla and Samsung SDI for batteries, SolarEdge and Ideal Power for inverters, and Landis+Gyr for smart meters and communications; but the announcement is light on the technical specifics of how these particular 
components will be integrated, as well as who will own the resource once it is installed on the grid. [18]

Consolidated Edison (ConEd), one of the largest investor-owned energy companies in the United States, is approaching energy storage in a different way. They are currently engaged in a demonstration project they have named the "Virtual Clean Power Plant", wherein 1.8 MW / 4 MWh of behind the meter battery-storage is paired with rooftop solar. [19]. ConEd has partnered with SunPower and Sunverge, who are providing a platform that aggregates control of the resources into a virtual power plant. There are more specifics in their outline about who will own the resource in the long-term than in the prior case. In ConEd's road-map for future work, the solar equipment and installation will be financed via a lease, with no upfront cost to the homeowner, and the storage equipment and installation would be owned by ConEd. The customer would then make monthly lease payments over 20 years for the rooftop PV installation, and also make monthly payments to for the resiliency services utility-owned storage system. This is similar to the proposed method for implementation of this thesis work; future researchers may want to find the most recent information about ConEd's success, or lack thereof, with their proposed business model.

In conclusion, we have seen that while academia is not short on proposed methods for integrating energy storage into the electric grid, they are often lacking in specifics of how their novel ideas would be implemented in practice. Predictions 
were presented for increasing growth in the distributed energy storage sector, as well as for decreasing battery capital costs. Also shown were proposed ways in which these trends could lead to increased value to the grid, if installed systems are dispatched for multiple services at one time. Finally, two examples of utilityrun demonstration projects were given, supporting Portland General Electric's investment in this research. We now move on to what we believe it would take to realize the proposed system of distributed ResBESS units. 


\section{General Design Considerations and Constraints}

This section discusses the design considerations and constraints of a conceptual ResBESS unit, compiled with the goal of informing field prototype construction and design. This knowledge was obtained through a combination of literary and industry research, conversations with PGE stakeholders, and laboratory experimentation. Additionally, consideration is given within this section to technical specifications for custom components that would need to be manufactured, were the project to be fully scaled into deployment.

\subsection{Future Meter Description}

Here, the proposed ResBESS meter functionality is discussed in detail. The differences from a typical meter are highlighted, and familiarity with a typical residential smart meter is assumed.

The ResBESS meter is envisioned to be the master control device for the individual ResBESS system: monitoring states, measuring power, issuing commands to the inverter, and communicating with the utility. Succinctly, the meter is to be the gateway through which the utility controls the ResBESS system. For this reason, significant detail is given within this section. A diagram showing the basic 
functional blocks within the conceptual ResBESS meter is shown on page 57 in Figure 4.1. This meter does not exist in practice, so it will need to be custom-built; for this thesis, an $\alpha$-prototype was built that implemented the desired functionality.

\subsubsection{Volt Connection}

The choice of connecting a utility-controlled, battery/inverter system at the meter of a single phase residential 240 Volt service - as opposed to the distribution-side voltage of $12.47 \mathrm{kV}$ - is the first consideration of this architecture. There are many advantages of this design compared to those proposed to date. These advantages include:

- This creates a customer value proposition and consequently a small revenue stream to partially justify the economics. As a resiliency option for the customer, this is a much "greener" and more reliable solution than those that rely on fossil fuel generation. Beyond a possible two-day energy supply the system could easily be modified to accommodate recharging from a portable generation device, e.g. a PHEV 5 .

- The inverters can be manufactured with the techniques used for mass market devices, thus possibly yielding a lower cost per kW.

\footnotetext{
${ }^{5}$ Plug-in Hybrid Electric Vehicle
} 
- While significant $\mathrm{NRE}^{6}$ in meter design is required, this would greatly reduce installation and maintenance cost.

- Used as a peaking resource, the location of this resource at the meter base could provide more than a $10 \%$ cost reduction per $\mathrm{kW}$ relative to central station peaking plants ${ }^{7}$ because of eliminating "capacity" lost in transmission and distribution line losses which can see half the total losses occur within the last 100 feet of power line.

\subsubsection{Ports, Points and Nodes}

The ResBESS meter will require three power ports ${ }^{8}$. In contrast to the two power ports found on a typical meter connecting a residence to the grid, the ResBESS meter requires a third power port to facilitate power transfer with the inverter. Because it is cost prohibitive to modify the meter socket to add this third port connecting to the meter, we propose adding this third port to the body of the meter itself.

This third port must also accommodate a data line point ${ }^{9}$ so that the meter and inverter may communicate with each other. Through this line, the meter will be able to control the inverter and monitor its state. This line will also pass through information regarding the state of the battery. The type of connection socket and the

\footnotetext{
${ }^{6}$ Non-Recurring Engineering: the one-time cost to research, develop, design and test a new product

${ }^{7}$ Information given by Director of Retail Technology Strategy at PGE, 2015

${ }^{8}$ Port: bi-directional power flow connection point

${ }^{9}$ Point: data-flow connection point
} 
cable are yet to be determined; a meter manufacturer will engineer an appropriate solution in collaboration with an inverter manufacturer.

The ResBESS meter must be capable of calculating power through each of the three power ports. A standard two-port smart meter need only monitor power using a single set of current transformers (CTs) and a single voltage sensor. For billing purposes in the proposed meter, power and accumulated energy (kWh) data shall be calculated for each of the three ports. This should be accomplished by using two sets of CTs on any of the three lines, with the current on the third line assumed through application of Kirchoff's Current Law, as well as a single voltage sensor positioned at the node ${ }^{10}$ of the three ports.

\subsubsection{Disconnect Switches}

For proper establishment of the various ResBESS system states, a disconnect switch shall be included at each of the three power ports. We recommend housing two of these - for the residence and utility connections - at the utility disconnect within the ResBESS meter, and locating the disconnect switch for the inverter port within the inverter. This switch at the inverter shall be controllable through the ResBESS meter and should also be cable of manual operation, featuring visible indication of its open/closed status and capable of being locked open.

The above switches use one of three options for disconnecting power to the house. The option detailed is a second disconnect switch inside the meter to enable

\footnotetext{
${ }^{10}$ Node: a junction of three or more lines
} 
the service disconnection function now achieved by some smart meters. This option may add considerable cost, volume, and another failure mode in the meter, and must be evaluated after discussions with a meter manufacturer. Secondarily, the home's service disconnect will always be present as required by the NEC, providing a preexisting method of residential disconnection. Finally, the traditional option of removal or replacement of the meter with a manual meter exchange is available.

\subsubsection{Communications and Control}

The ResBESS meter shall have more processing power, memory and communications capabilities than those of a typical smart meter. The meter shall not only monitor and calculate power through the three power ports, but it shall also control each of the three disconnect switches. In addition, the ResBESS meter will send and receive data with the utility more often than is typical, since the utility could be issuing frequent commands and polling for data often. Incorporation of these capabilities will incur additional costs, but the incremental costs are thought to be very small compared the system cost.

Within this context, the need for secure and reliable control and metering telemetry with a system of ResBESS units is paramount. The physical and cyberprotection of certain devices deemed vital to the reliability of the bulk electric system (BES) is governed by NERC ${ }^{11}$ and their Critical Infrastructure Protection

\footnotetext{
${ }^{11}$ The National Electric Reliability Corporation, under the authority of the Federal Electric Regulatory Commission (FERC)
} 
(CIP) rules. These rules may apply to ResBESS, and are discussed further in Appendix E.2.1.

\subsubsection{Statement about Requirements of IEEE 1547}

IEEE 1547 is the prevailing industry standard for guiding interconnection of distributed resources (DR). It sets standards for interconnection between an electric power system and DR owned by another party. Generally, this standard is used by regulating agencies such as public utility commissions to protect the power grid from poorly designed or connected DR that are not utility owned. Since ResBESS units will be owned and controlled by the utility, IEEE 1547 need not apply. Regardless, some aspects of IEEE 1547 are worth applying to ResBESS. Appendix D discusses the scope and purpose of IEEE 1547 and outlines its various subsections.

Unless stated otherwise within this document, it is recommended that ResBESS operation adhere to the specification established by IEEE 1547-2003. It must be noted as well that IEEE 1547 is an evolving standard, influenced particularly by grid stability issues related to increased penetration of renewables and other distributed assets. As such, the influence of 1547-related issues on the ResBESS design will evolve as this project continues to develop. 


\subsubsection{Control Telemetry}

The desired method for transmitting control commands to ResBESS units is a mass broadcast signal that all units would receive; an $\operatorname{RBDS}^{12}$ sideband signal may be the preferred means to accomplish this, as PGE already owns some FM spectra. Use of this method of control for DR is not new; it was studied for use in a system of Programmable Communicating Thermostats in California. [20] Regardless of the the control protocol used in a system of ResBESS units, it must allow for the ability to intend a single broadcast message to cause action by a single unit, a group of units, or all units. Additionally, each individual ResBESS unit must be able to be assigned over the broadcast network as a member of multiple groups, as required for system control.

As an example of how securing these broadcasts could be handled by future researchers, a paper entitled Securing RDS Broadcast Messages for Smart Grid Applications, outlines several methods of encryption which "...provide strong authentication against attackers who attempt to forge signatures without knowledge of private keys (which are held at the transmitter)." Furthermore, "[t]he information exposed in the transmitted messages will not help an attacker in forging future messages...as messages are time-sensitive and the senders and receivers in the network coarsely time-synchronized, replay-attacks are prevented as well." [21]

In its most simple form, a transmitted supervisory command would be sent to all

\footnotetext{
${ }^{12}$ Radio Broadcast Data System: a name used in the US for the IEC 62106:2015 communications protocol, governing the embedding of small amounts of digital information in conventional FM radio broadcasts
} 
units concurrently, thereby controlling all units as one aggregated group. However, since it is may also be desirable to send commands to subgroups of units or even to single units, we propose that the broadcast signal should have a header containing a 32-bit addressing word. This word would provide identification information so as to specify which units or groups of units are required to obey the command being sent. While 32 bits may seem large, a 32-bit word was chosen because IEEE 1547.3 section 4.4 encourages extensibility: "Use cases and stakeholder needs are bound to evolve. For this reason, all aspects of monitoring, information exchange, and control (MIC) systems should be extensible."[22, p. 15] Use of $2^{32}$, or 4.3 billion unique addresses, would permit easy addressing of both individual units and subgroups within a large balancing area via a single broadcast signal at an even larger scale than the system proposed. Note that a mass reply from all 25,000 units, where each unit includes its respective 32 bit address, would add $100 \mathrm{kB}$ of data to the reply.

\subsubsection{Metering Telemetry}

With regards to metered data that the utility would desire to receive from the ResBESS network, IEEE 1547.3 section 4.5.1 recommends that "...automatic configuration [use] self-description (also called interrogation)", which requires "...a device to describe itself in a standard way upon request by other DR devices or a central controller."[22] To adhere to this recommendation, when queried, each unit should have the information requested by Balancing Authority or any other department ready to send in a pre-formatted, standardized way using an industry accepted 
common information model (CIM) - more information about CIM is included in Section 4.1.4.4.

It may be desirable to prevent 25,000 telemetry messages returning to the utility simultaneously. If this were required, we envision a system that uses a random time-spaced query of select units to achieve a statistical model of the system as a whole; regardless, each unit should additionally be able to provide state-of-charge, $\mathrm{kWh}, \mathrm{kW}$, operational ability (is the battery nominal, is the inverter nominal, is the meter working, etc), and so on. These selected data should be programmable by the utility and not locked into the software or firmware of the meter.

If simultaneous delivery of all metering telemetry to the utility is desired, it may still be beneficial to keep the overall data transfer as small as possible. It is difficult to conjecture at this point what the total size of data transferred would be in future, but the amount of data currently passed to the utility within the laboratory prototype is summarized in Section 5.3.3 to this end, and consideration is also given to this topic in Section 4.2.4.

Finally, current AMI meters employ an IPv6 RF Mesh to allow the utility to obtain metered data (which we recommend continuing to use), and these meters are additionally secured to NERC CIP standards [23]. This bodes well for future researchers desiring a custom-built meter for the project. The protocol used to communicate data between PGE and ResBESS units could be DNP3 ${ }^{13}$ or IEC 61XXX as recommended above. In lieu of using the AMI network, 3G or 4G cellular signals

\footnotetext{
${ }^{13} \mathrm{DNP} 3$ is the preferred legacy protocol used by PGE
} 
are also possibilities, though they are not as secure.

\subsubsection{Statement about IEC Common Information Models}

There are two prevailing standards we recommend: IEC 61970 - CIM for Energy Management, or IEC 61850 - CIM for Power Utility Automation. IEC 61850 is an information model for substation and feeder equipment, while IEC 61970 is an information model of a power system as seend from a control center viewpoint. The standard of IEC 61850 focuses upon information models and information exchange, creating ways to group related data objects for either equipment status or measurement; essentially allowing devices to self-describe to a controller. IEC 61850-7-420 is a newer extension to 61850 which covers CIM for distributed energy resources including energy storage. Tying 61850 back to the control room is IEC 61970, which includes an application programming interface (API), allowing many different devices' CIM to be brought together in an energy management system (EMS) environment for a utility's central system controller. IEC 61970 can import the information produced or modeled within 61850 into 61970, which includes CIM packages such as $\mathrm{SCADA}^{14}$, energy scheduling, financial, and other packages which would help realize the full potential of ResBESS integration into existing utility operations.

\footnotetext{
${ }^{14}$ Supervisory Control And Data Acquisition
} 


\subsubsection{Neutral Connection}

The ResBESS meter must pass through a connection from the neutral line within the meter socket to the inverter. This is an unusual specification for a smart meter. 240 VAC inverters must have access to the residence neutral line to establish a reference point for the two 120 VAC supplies. In order to avoid running a separate conduit and neutral line between the inverter and the meter socket or service panel, we recommend providing the inverter with access to the neutral line through the meter, if possible, to help keep installation costs low. 


\subsection{Desired System States and Transitions}

We propose five system states for ResBESS units, defined below. Transitioning between these states involves actuating combinations of the three disconnect switches mentioned previously. The state transition diagram in Figure 4.2 shows four of these states, as well as the switch status changes that must occur to transition between states. To preserve clarity, the No Service (NS) state has been omitted from the diagram. More detail on the state transition diagram is provided in Subsection 4.2.3

\subsubsection{System State Definitions}

- Grid Tied (GT): All connections between utility, residence and inverter are closed. Power may be directed to or from the battery by the utility as needed. The residence is served by the utility and/or the battery.

- Backup (BU): The residence and inverter connections are closed, while the utility connection is open. Power cannot be directed to or from the batteries by the utility. The residence is served exclusively by the batteries.

- Maintenance (MT): The residence and utility connections are closed, while the inverter connection is open. Power cannot be directed to or from the batteries by the utility. The residence is served exclusively by the utility.

- Service Disconnect (SD) [only available if residence disconnect is chosen in meter]: The utility and inverter connections are closed, while the residence 
connection is open. Power may be directed to or from the batteries by the utility. The residence is served by neither the grid nor batteries. Note, this state would not be implemented if the decision is made to not include the residential disconnect switch within the system design.

- No Service (NS): All disconnect switches are open. This state occurs when any two of the following three situations are true: utility service is unavailable; residence is not to be served by the utility or battery; battery or inverter requires maintenance. Power transfer may not occur between any of the three entities. 


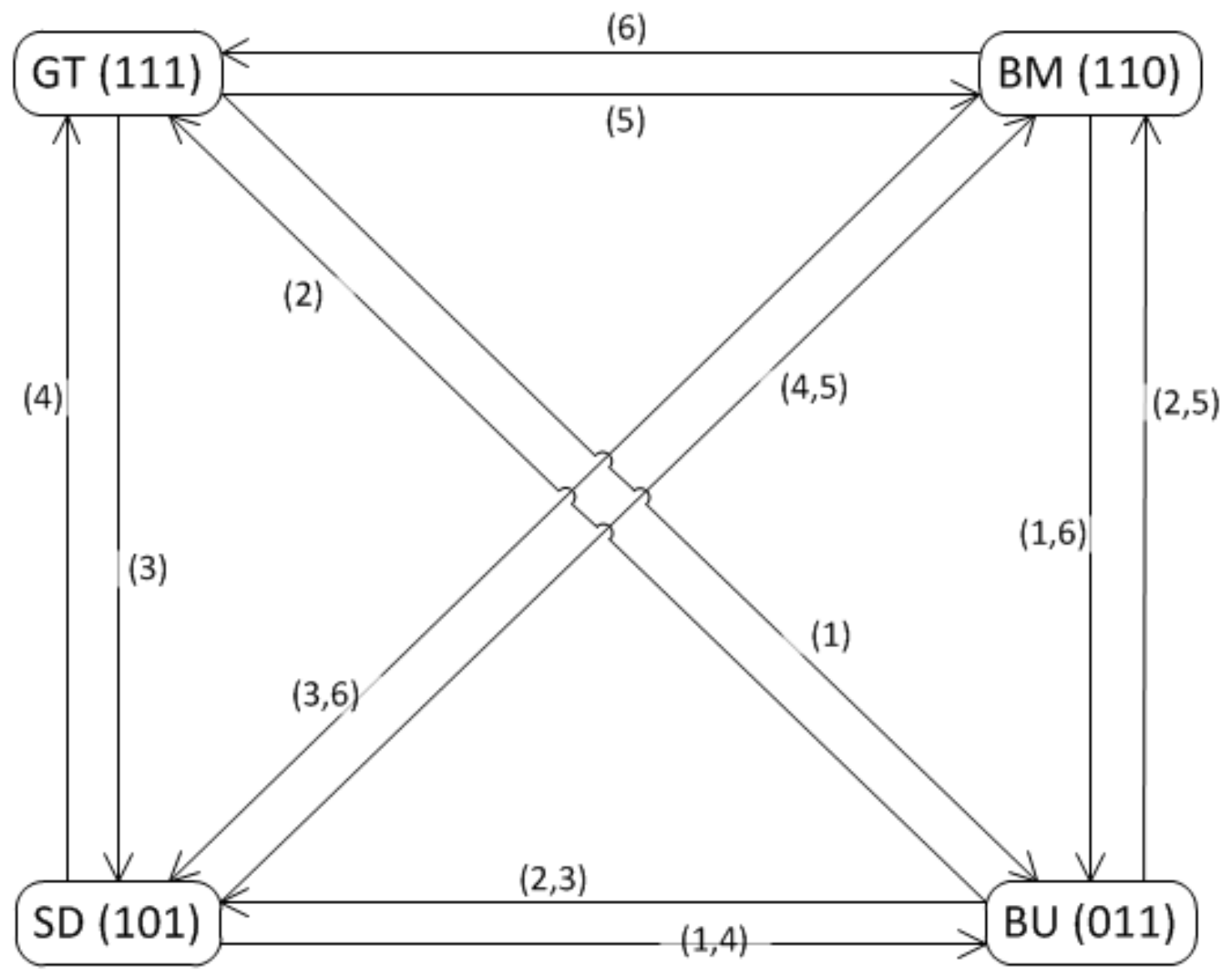

\section{LEGEND}

\section{Transition Cause}

1) Connection to utility opens

2) Connection to utility closes

3) Connection to residence opens

4) Connection to residence closes

5) Connection to inverter opens

6) Connection to inverter closes

\section{State of Disconnect Switches (xxx)}

Three bit code: state of utility, residence and inverter switches.

1 - closed, 0 - open

Figure 4.2: The state transition diagram for four ResBESS basic use cases. Transitioning between one state and another requires opening and/or closing combinations of the three disconnect switches. 


\subsubsection{Methods of Switching}

Switching between states shall be accomplished using two methods: autonomously via commands from the ResBESS meter, and remotely via direct utility command. It is unlikely that the latter method will be used frequently for these basic cases. However, the utility shall retain the ability to control individual units or groups of units remotely. For instance, there may be a need to disconnect residences delinquent on utility bill payments, or to disable ResBESS units that have flagged internal system errors.

The system will operate in more than one mode as permitted by utility operations. For example, while the system will normally be permitted to autonomously change modes from grid-tied operation to premises backup mode in the event of a grid outage, the reverse is not true. That is, when grid power is restored, resuming grid-tied operation must normally be preceded by a command from the distribution operations center.

The state transition from Grid Tied to Backup and vise-versa should adhere to specifications established by IEEE 1547-2003, which was introduced in Subsection 4.1.4.1. The specification that the "The DR [distributed resource] shall not energize the Area EPS [electrical power system] when the Area EPS is de-energized" is among the most fundamental of these guidelines that should be considered. [24, p. 10] However, it is likely that some other 1547 specifications may not be applied to ResBESS, such as "...[w] hen the system frequency is in a range given ... the DR 
shall cease to energize the Area EPS within the clearing time as indicated", since strict adherence to this particular specification would not allow a ResBESS unit to provide frequency regulation. [24, p. 8]

\subsubsection{State Transition Switching Logic}

The switching logic that a ResBESS meter should use to determine the state transitions was outlined by the state transition diagram seen in Figure 4.2. With the five states proposed, twenty transitions are possible. Recall that transitioning between states involves actuating one or more of the three disconnect switches.

In summary, states - defined in Subsection 4.2.1 - shall be determined through monitoring of five status signals, which we propose and define for in Subsection 4.2.3.1; and, as shown in Figure 4.2, a system state can be simply defined by a three bit code indicating the switch positions of the utility, residence and inverter disconnect switches. All of these definitions and relationships are summarized in the truth-table found in Table 4.1, and boolean logic scenarios for all of the transitions between states are provided in Appendix G.5.

\subsubsection{Status Signals Definitions}

Inverter Operable (Inv) A signal relayed from the inverter to the ResBESS meter. Is FALSE when the inverter is subject to a fault condition or when the manual disconnect switch has been actuated. Always works in conjunction with 'Battery Operable' in an AND function. 
Battery Operable (Bat) A signal relayed from the battery to the ResBESS meter through the inverter. Is FALSE when the battery is subject a fault condition. Always works in conjunction with 'Inverter Operable' in an AND function.

Nominal Service (Nom) A signal controlled by the utility. Informs ResBESS unit that normal utility service to the home should be provided. Is False to indicate service should be disconnected. (Status only meaningful if the optional house disconnect switch exists with the meter.)

Grid Steady-State (GSS) Signal produced by the ResBESS meter. Is FALSE when the meter detects abnormal steady-state status (voltage and frequency check) at the point of common connection. Always works in conjunction with 'Grid Online' in an AND function.

Grid Online (GO) - A redundant check signal to 'Grid Steady State', which is controlled by the utility. This provides the utility with a means to confirm grid status to ResBESS units. Is FALSE if the system operator has determined ResBESS units should not be online. Always works in conjunction with 'Grid Steady-State' in an AND function. 


\begin{tabular}{ccc|ccc|l}
\hline \hline \multicolumn{2}{l}{ Status Signals } & \multicolumn{4}{c}{ Switch Positions } & \multicolumn{1}{l}{ System State } \\
\hline Inv $\wedge$ Bat & Nom & GSS $\wedge$ GO & Utility & Residence & Inverter & \\
\hline 0 & 0 & 0 & 0 & 0 & 0 & No Service \\
0 & 0 & 1 & 0 & 0 & 0 & No Service \\
0 & 1 & 0 & 0 & 0 & 0 & No Service \\
0 & 1 & 1 & 1 & 1 & 0 & Maintenance \\
1 & 0 & 0 & 0 & 0 & 0 & No Service \\
1 & 0 & 1 & 1 & 0 & 1 & Service Disconnect \\
1 & 1 & 0 & 0 & 1 & 1 & Backup \\
1 & 1 & 1 & 1 & 1 & 1 & Grid Tied \\
\hline
\end{tabular}

Table 4.1: Truth table for the Status Signals and the resulting Switch Positions and Systems States. Note two sets of signals are AND $(\wedge)$ combinations, $I n v \wedge$ Bat and GSS $\wedge$ GO.

\subsubsection{Utility Control and Data Handling}

In the proposed system state logic, only a single bit out of five status signals is required to be transmitted by the utility to control the connection of each ResBESS unit to the grid, which means only 3.125 kilobytes would be necessary to connect or disconnect all units in a scaled system. Additional thought on the amount of data which would need to be passed from utility to ResBESS units and vice versa is detailed in this section.

Additionally, in a fully-deployed scenario, a system operator would need to be able to view information about tens of thousands of ResBESS units in aggregate rather than just individually. The operator would also need to be able to control many of these units in aggregate rather than at the granular level of a single unit. Thus, a conceptual graphical user interface (GUI) for a utility system operator is also discussed in this section. 


\subsubsection{System Overview}

The System Overview screen, shown in Figure 4.3, could provide the operator with information pertinent to a large bank of batteries, consisting of many thousands of ResBESS units. In this screen the operator may view the number of units both online and offline within the selected bank, the charge or discharge status of the bank, and the nameplate rating of the bank. The operator would be able to control the charging and discharging of the bank.

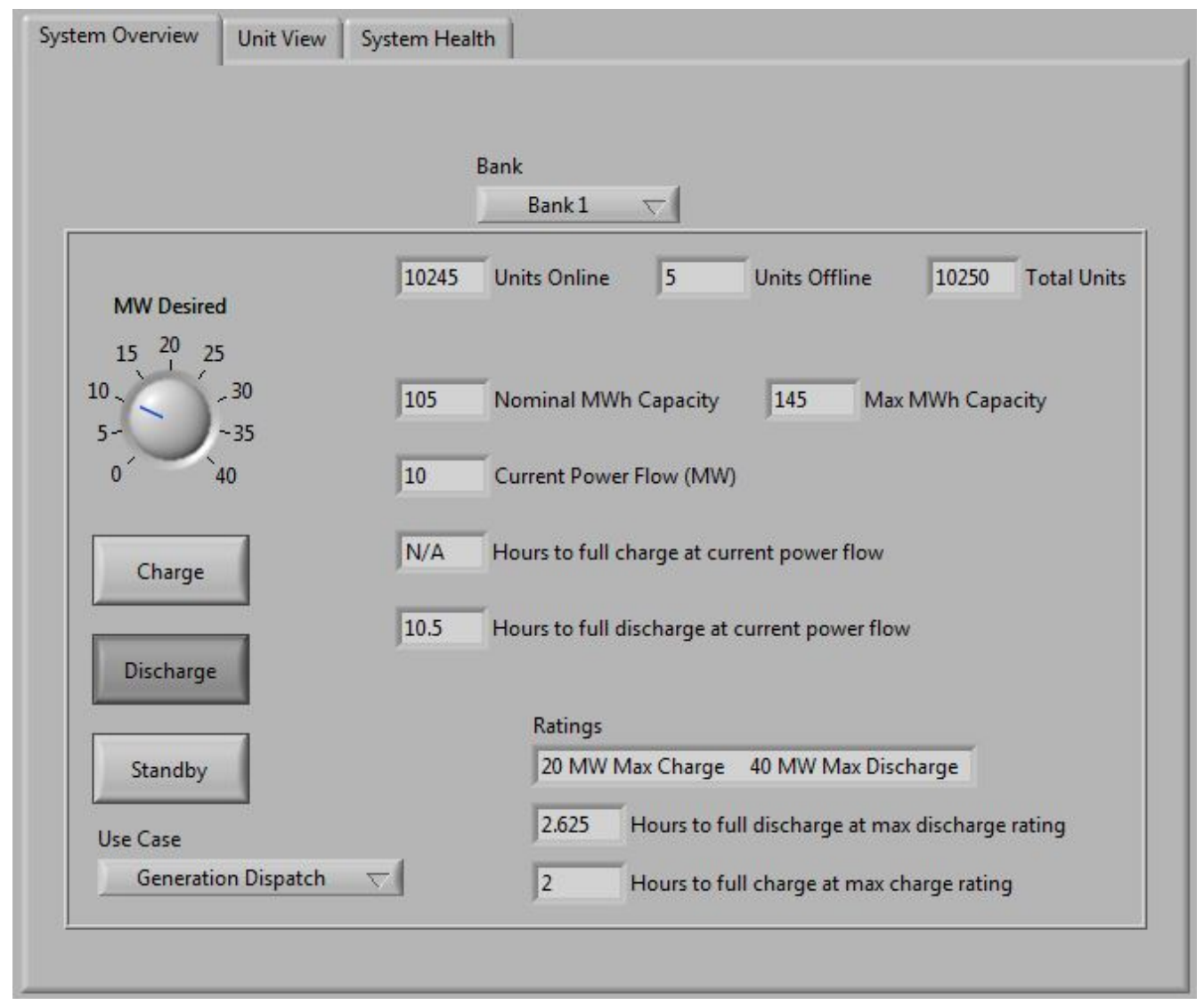

Figure 4.3: System Operator view showing the overview of the system.

To accomplish this, a method of determining online and offline units must be created. The status bits outlined in Section 4.2.3.1 would provide such a method. 
Were these bits combined into a 16 bit error code (16 bits would allow extensibility for additional system states or errors), the utility could easily ascertain the health of each unit using $150 \mathrm{kB}$ of data per total-system-health transmission: $50 \mathrm{kB}$ for $16 \times 25,000$ health bits, and and $100 \mathrm{kB}$ for $32 \times 25,000$ ID addressing bits. ${ }^{15}$

To accomplish the control which is seen in this screen - specifically MW-rate control, and charge/discharge/standby determination - additional data would need to be broadcast. Only a few bits would be needed for operational control: a single byte would allow for 256 possible operational states to be assigned, and 32 bits would allow for commands to be addressed to all units, or a group of units; but the amount of data for determining rate of charge/discharge, or any other numerical properties of operation (e.g. cut-in frequency, if units were configured as frequency regulating devices), would be determined by the precision the utility would desire these properties to have.

\subsubsection{Unit Overview}

The Unit Overview screen, shown in Figure 4.4, provides the operator with information regarding an individual ResBESS unit within the bank. In this screen they could view the physical location of the unit, the date of installation and the distribution equipment to which the unit is connected, if the utility chose to detail thins information. This screen could also display the operating state and error

\footnotetext{
${ }^{15}$ Mentioned in Subsection 4.1.4.2
} 
status of the unit. The operation state and the nameplate rating of the unit are also shown.

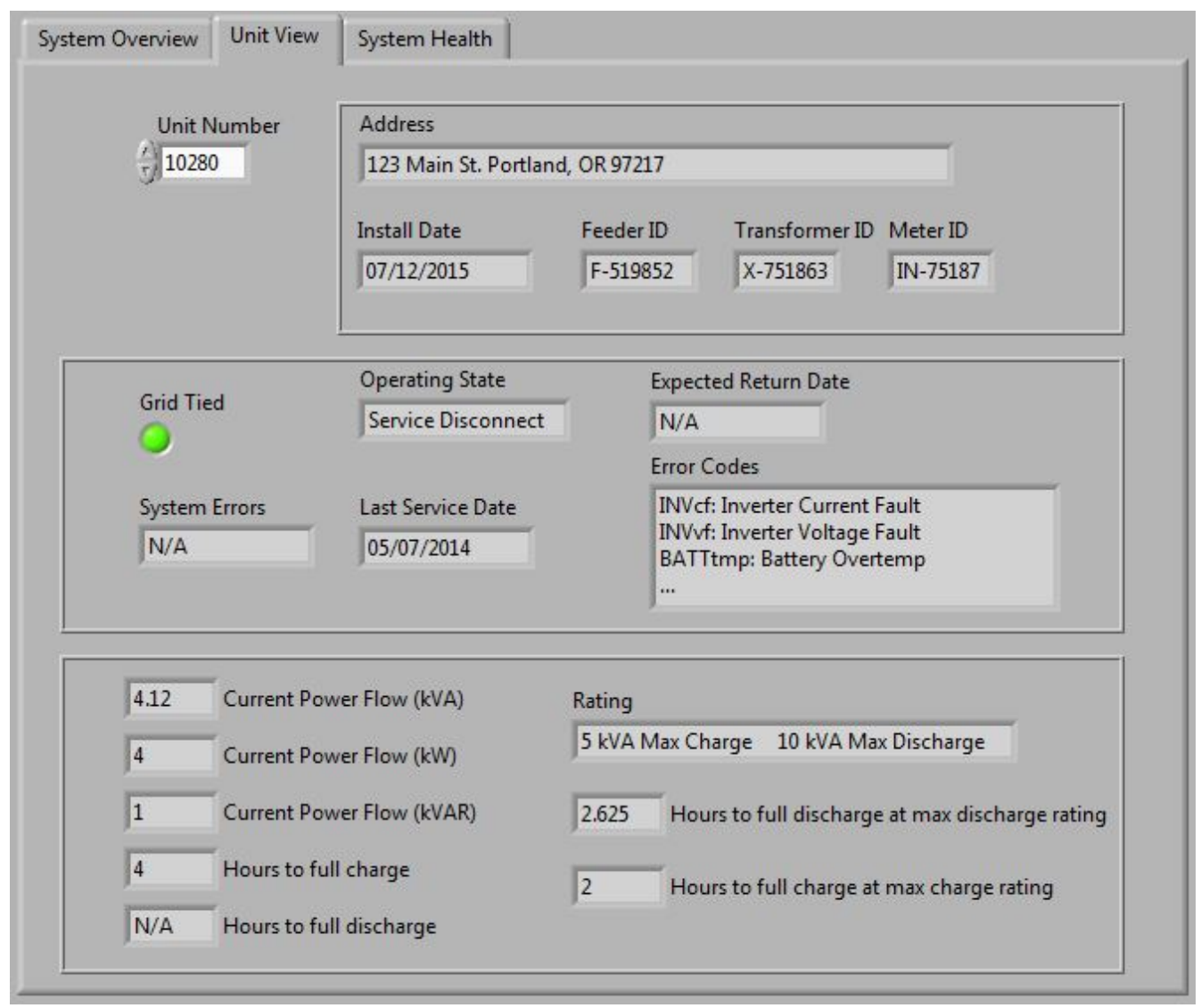

Figure 4.4: System Operator view showing the details of an individual unit.

The data received from each unit in the conceptual GUI includes kVA, kVAr, and $\mathrm{kW}$ as well as calculations about SoC. If these data were each 16 bits, and were reported by each of 25,000 units, then $200 \mathrm{kB}$ would be received per transmission. In summary, a rough estimate for returned data from the aggregated system would be $350 \mathrm{kB}$ per received data block: $150 \mathrm{kB}$ for each aggregate system-health data transmission, and $200 \mathrm{kB}$ for each aggregate system-metered-data transmission. 


\subsubsection{System and Unit Health}

Finally, the System Health screen, shown in Figure 4.5, might give an operator the ability to view various lists containing work orders for systems in need of maintenance. These lists could be automatically updated by service technicians and might include categories such as 'Units Not Available for Service,' 'Units with Maintenance Today,' 'Units with Unreliable Communication,' and 'Unit Work Orders.' Clicking on any of the unit IDs could switch the screen to the Unit Overview of the selected unit.

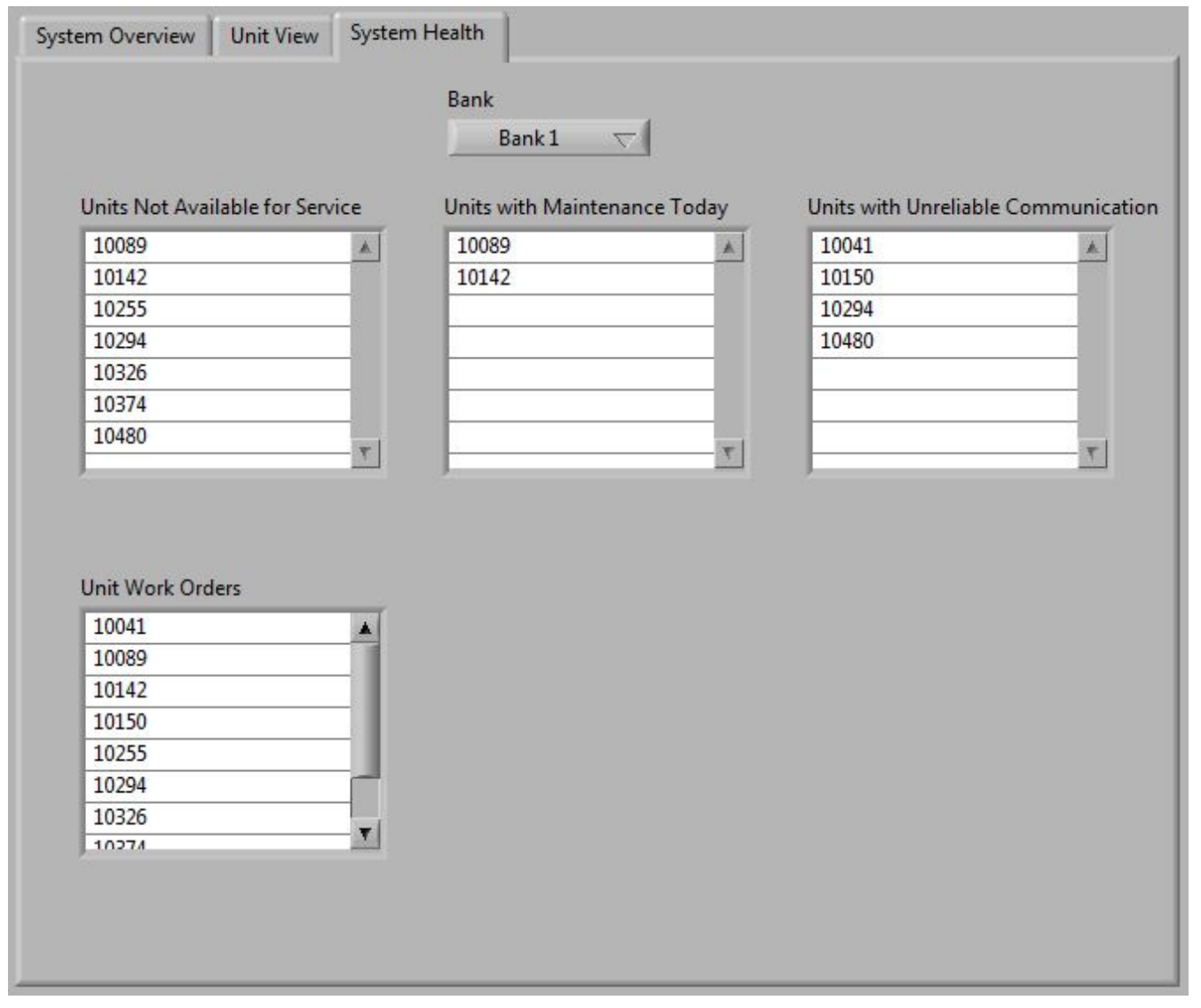

Figure 4.5: System Operator view showing the health of the system.

To achieve the ability for the utility to have this system and unit health consid- 
eration, we propose some options for removing and re-entering units into service. As a first concern, communications should be synchronized or scheduled between the utility and the system such that if an expected communication is not received, an instant categorization as unreliable can be made. These units need not be completely removed from service; for instance, if the last received data show total system health, then it is possible that the unit will respond to a command in future. However, it would be imperative to remove the unit from any numerical commitments to critical services due to its unreliability, as well as flag it for a check-up by maintenance crews.

This communication synchronization could occur by tying the utility and the aggregated system together through a "heartbeat", or simply by detecting if a signal has disappeared: e.g., in the case of an individual unit, it should see the utility's Grid-Online status bit at all times, and thus could remove itself from service if that status bit were not seen; whereas in the case of the utility, loss of communication could be determined simply examining returned unit health and metered data for missing or corrupt unit data (each of which should be identified with a unique 32-bit address).

For returning a unit to service, repair technicians would need to access the local data of each device to ascertain the exact cause of the issue, as well as to reset any status bits which were triggering a removal from service.

In all of these considerations - system health and control, unit health and 
control, and the data amount and handling necessary - future work remains to fully describe and realize this discussion.

\subsection{Use Cases}

As detailed in the previous section, the five system states proposed describe the status of a ResBESS unit as pertains to its disconnect switch configuration. ResBESS will likely be in the Grid Tied state for the majority of the time, so long as the utility provides electrical service. ResBESS should transition out of that state only if the utility service fails $\{G T$ to $B U\}$; or if the battery or inverter encounter an internal system fault and therefore require maintenance $\{G T$ to $M T\}$; or if the customer becomes delinquent on billing $\{G T$ to $S D\}$; or combinations thereof $\{G T$ to $N S\}$.

When operating in the Grid Tied system state, there are several specific ways that the utility could utilize ResBESS units, and these use-cases are what provide the utility with value. This section outlines these use cases, the reasons that ResBESS units would be desirable to use in these cases, and some of the technical details pertaining to each case.

\subsubsection{Use-Cases Defined}

As proposed, a utility with a customer base similar to that of PGE's would employ around forty to fifty thousand units within its balancing area, providing a nominal nameplate capacity of between 290 and $360 \mathrm{MW}$. Through the remote broadcast control suggested, or through distributed intelligence, these units could 
be aggregated to act as one single resource, or several smaller resources. Ideally, the ResBESS units would act in concert such that the aggregate resource could provide services for the utility. These services could include frequency regulation, contingency reserves, peak shaving, renewables integration, economic arbitrage and volt/VAr support. Aggregated ResBESS units could be configured to provide one or more of these services concurrently. Here, these services are listed and examined specifically within the context of the proposed system.

\subsubsection{Frequency Regulation}

Peak Reliability ${ }^{16}$ requirements mandate that frequency be maintained within a tight window, $59.932 \mathrm{~Hz}$ to $60.068 \mathrm{~Hz}$ - any deviation from these bounds which lasts longer than 5 minutes requires immediate action. Yet, changes in load and variations from non-dispatchable generation resources (PV and wind, specifically) both contribute to frequency deviations. Frequency regulation is a fast-acting automatic service, typically provided by the governor response of generators, that can act to stabilize system frequency. Resources that provide frequency regulation must be able to react within less than 15 seconds and must remain available for up to one minute.

Because of the fast ramp-up rates of BESS systems, ResBESS units could be used to provide frequency regulation. Utility-interactive inverters are capable of ramping up to rated power in one second or less.

\footnotetext{
${ }^{16}$ Peak Reliability operates as the Western Interconnect's reliability coordinator under the authority of the National Electric Reliability Corporation (NERC)
} 


\subsubsection{Contingency Reserve}

There are several varieties of contingency reserves, but two of them are focused upon here: spinning reserves, and non-spinning reserves (frequency regulation is also considered a form of contingency reserve). Spinning and non-spinning contingency reserves are services that can accommodate large unexpected system events such as the unplanned loss of a significant generator or disconnect of a major transmission line. These reserves are brought online to maintain system balance.

Spinning reserves are online generation, storage, or responsive load resources that are synchronized, ready to begin responding immediately and fully responsive within 10 minutes of a dispatch instruction.

Non-spinning reserves are off-line generation, storage, and responsive load resources that are held in reserve but not synchronized. These can be ramped to capacity and synchronized within 10 minutes of a dispatch instruction.

Both of these reserves are moderately quickly available resources, required to respond within 10 minutes. They must maintain their support for up to 105 minutes, long enough for replacement reserves to become fully operational.

Aggregated ResBESS units meet the requirements for both spinning and nonspinning reserves. Grid-tied inverters are synchronized at all times ("spinning" reserves need not carry inertia) and have sufficient ramp rates to meet dispatch requirements. Regarding capacity, 8 kVA ResBESS units with $52.8 \mathrm{kWh}$ batteries could remain online at rated power for 6.6 hours; even discharged to $26.5 \%$ state of 
charge, ResBESS units would be able to meet the 105 minute capacity requirement.

\subsubsection{Peak Shaving}

While frequency regulation and contingency reserve services are reactionary services, peak shaving may be considered a proactive service. Peak shaving involves using storage resources to shape the load profile throughout a day, with the specific goal of reducing demand on generators during the peak loading hours of the day. This is an interhour service, often referred to as 'shaping.' Storage systems, such as ResBESS, are charged during low demand periods, typically during the early morning hours, or when nondispatchable generation comes online, such as the evenings for wind. During peak demand hours, this stored energy is discharged, thereby reducing the demand on traditional generators. Such shaping is beneficial in that it reduces the number of idle generators that need to be maintained to serve peak load, thereby saving capital and O\&M costs on non-revenue-generating assets.

\subsubsection{Renewables Integration or Firming Services}

Renewables integration is the use of a dispatchable generation or storage resource to accommodate unscheduled fluctuations from renewable generators, specifically wind and PV. The need for renewables integration services stems from resource forecast error, an inevitable issue when integrating stochastic generation sources. Wind may be forecast to blow at a particular time, but may actually arrive earlier 
or later than forecast. Likewise for PV, insolation ${ }^{17}$ could suddenly decrease due to cloud cover, then return just as rapidly. In both cases, these unscheduled variations in power availability affect a utility's dispatch schedule, which is considered in terms of intrahour, hour-ahead or day-ahead. Failure to meet commitments during these periods, either long or short, would force the utility to turn to the energy spot market, run generation plants in sub-optimal modes, or incur financial penalties from the system regulator for failing to follow the dispatch schedule. A resource such as ResBESS can help bridge the difference between scheduled and generated renewable resources. ResBESS units would be available to provide incrementing and decrementing services (inc/dec), as well as to absorb excess generation during periods of low load and high renewables generation.

\subsubsection{Volt/VAr support}

Any voltage source converter (VSC) can be used to manage reactive power. VSCs are the power electronics subsubsystem within inverters, STATCOMs, UPFCs and VSC-based HVDC converters ${ }^{18}$. In addition to providing real power, VSCs can concurrently inject or absorb reactive power. The inverters within ResBESS units could therefore be designed to provide volt/VAr services, providing voltage support, power factor correction (PFC) or conservation voltage reduction (CVR) on their local feeders.

\footnotetext{
${ }^{17}$ Insolation is the solar radiation that reaches the earth's surface, measured in $\frac{W}{m^{2}}$ or $\frac{k W h}{m^{2} * d a y}$

${ }^{18}$ As opposed to line-commutated HVDC converters, such as BPA's system at Celilo
} 
Major manufacturers of inverters such as $\mathrm{ABB}$ and Enphase have begun to include this feature within their MW-scale inverters and select $\mathrm{kW}$-scale inverters. Utility-owned and -controlled assets such as ResBESS, not being subject to IEEE 1547.7, could be used as a distributed volt/VAr support tool through a utility's balancing area. Providing reactive power support does impact an inverter's real power output, since the inverter is limited by its VA rating. But the orthogonal relationship between real and reactive power means that an inverter can provide significant volt/VAr support while still managing real power near its maximum VA rating. In other words, ResBESS units can be used to provide the aforementioned system-wide energy services while concurrently providing local volt/VAr support.

\subsubsection{Economic Arbitrage}

With economic arbitrage, energy prices are used to influence decisions of when to charge and discharge a storage resource. Effectively, this provides an economic means for providing both firming and shaping services, such as for peak shaving and renewables integration. Understanding that energy is inexpensive during low demand periods and when non-dispatchable generators come on line at inconvenient times, and that it is expensive during peak demand periods, the strategy of "buy low, sell high" leads to peak shaving, as batteries are charged in the early morning hours and discharged during peak demand. Having a system like ResBESS available allows a utility to perform economic arbitrage. The utility may hedge against high spot market prices by taking advantage of under-utilized generation 
resources during low demand periods. Likewise, a large-scale storage system would allow the utility to sell into the spot market its excess energy reserves during peak demand periods, thereby providing an additional revenue stream.

\subsection{Use-Case Motivations}

Tens of thousands of aggregated ResBESS units may be configured to provide any combination of these services. Subgroups of ResBESS units may be set aside to provide specific services, with a fraction dedicated to frequency regulation, others set aside as contingency reserves and still others focused on economic arbitrage. Concurrently, all units could be providing local volt/VAr services like CVR or PFC. The fast response time of ResBESS units means that the system could be reconfigured quickly. For instance, units dedicated to economic arbitrage could be rapidly deployed to provide spinning reserves if need arose.

The ability to store energy is a revenue opportunity as well as a tool for maintaining reliable balancing services. At present, only pumped hydro (PES) has the ability to provide these storage-related energy services. However, PES tend to be large resources located far from load centers. On the other hand, a ResBESS system would be comprised of storage resources distributed throughout the balancing area. Under direct utility control, a ResBESS system would provide multiple energy services without incurring transmission line losses or charges, while concurrently providing local volt/VAr services. 


\subsubsection{Use-Case Technical Needs}

In order to inform PSU's research, time was spent with multiple stakeholders from within PGE, gathering their feedback to better inform recommendations. The requirements suggested by these stakeholders helped define the technical abilities the ResBESS units must possess. Meetings were held with PGE stakeholders from Distribution Engineering, System Control Center, Dispatch, Balancing, GenOnSys (Distributed Standby Generation), Meter Services and Power Operations.

Using their feedback, lists of requirements for some of the use-cases described above were developed. The requirements for each use-case are as follows:

\section{Frequency Regulation}

- telemetry sent/received every $4 \mathrm{sec}$

- resource must be available in $4 \mathrm{sec}$

- resource must stay available for $1 \mathrm{~min}$

- need to know available capacity MW of resource

- need to know MWh of resource after-the-fact each hour

- resource needs to automatically respond to frequency deviations

\section{Spinning Reserve (as part of Contingency Reserves)}

- telemetry sent/received every $4 \mathrm{sec}$

- resource must be fully loaded within 10 min of request

- resource must stay available for $60 \mathrm{~min}$ or more

- need to know available capacity MW of resource 
- need to know MWh of resource after-the-fact each hour

- resource needs to be able to be dispatched by the Balancing Authority

\section{Generation/Load Resource "load shaping"}

- telemetry sent/received every $60 \mathrm{sec}$

- resource must be available in $15 \mathrm{~min}$

- resource must stay available for $60 \mathrm{~min}$ or more

- need to know upward (generation) and downward (load) capacity MW of resource

- need to know MWh of resource after-the-fact each hour

- resource needs to be able to be dispatched by the Balancing Authority

If the ResBESS system is designed to meet the requirements for frequency regulation and spinning reserves (response speed and energy capacity, respectively), then the system would meet the requirements for all other energy services use-cases as well. The response time requirement - available in 4 seconds or less - is certainly manageable. Utility-scale inverters currently on the market have a pre-set ramp rate of $20 \%$ rated power per second, resulting in $80 \%$ resource availability after 4 seconds; however, this ramp rate is field-programmable, with a maximum ramp rate of up to $100 \%$ per second ${ }^{19}$. ResBESS units would need a ramp rate of just $25 \%$ per second to meet the 4 second requirement, certainly feasible with current VSC technology.

\footnotetext{
${ }^{19}$ Personal conversation with Advanced Energy powerr systems engineer Don Sweeny, 2015
} 


\subsection{Technical Specifications}

Based on the constraints and considerations discussed in this section, specifications were developed for both the meter and the inverter, intended for use by a potential manufacturing partner in future. These technical specifications are listed in Appendix C.1 and Appendix C.2, respectively. 


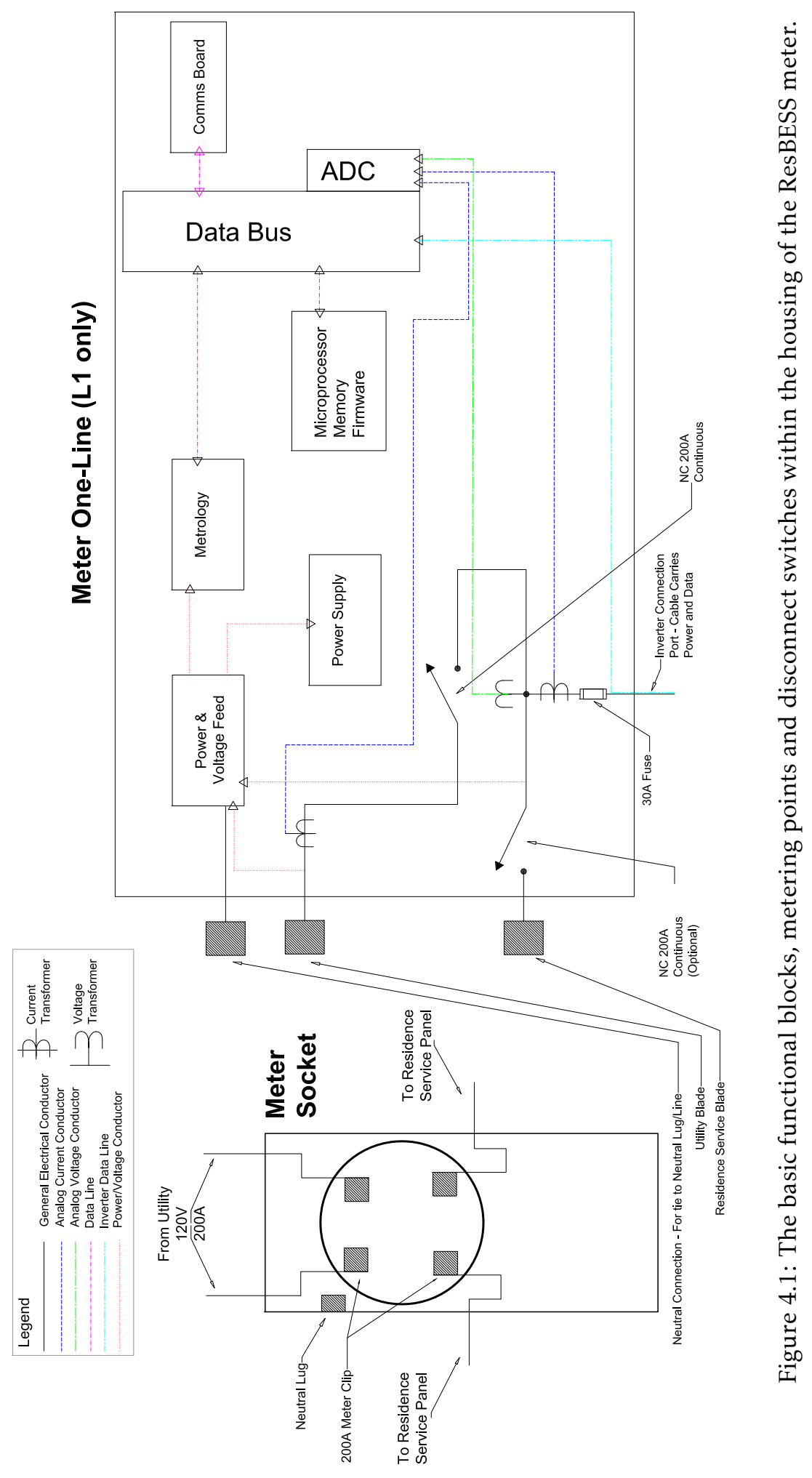




\section{Prototype Design Considerations and Constraints}

As alluded to in prior sections, the three major components of the desired ResBESS system - battery, inverter, and meter - do not currently exist. Therefore, it was desired to identify constraints that would be introduced to future researchers who would be working with currently available, off-the-shelf devices. In this section, the devices used in the laboratory prototype - and the thoughts that led to their acquisition - are summarized, and lessons learned from the prototype are discussed.

\subsection{Battery}

To make this vision successful, we desire affordable, safe, inert, and low maintenance batteries. These units must require very little maintenance in order to avoid the need for frequent service visits; homeowners would not be amenable to repeat visits from the utility for battery maintenance. Also, materials within the units must be safe to store within customers' homes, presenting little danger to residents in the case of system failure.

\subsubsection{Costs}

This component will comprise the bulk of the ResBESS unit's cost; however, costs for batteries are dropping, and those of some battery technology families are now 
approaching $\$ 300 / \mathrm{kWh}$. When costs reach $\$ 200 / \mathrm{kWh}$ they can be an economic resource which would complement new and existing utility supply-side capabilities.

Nykvist and Nilsson compiled capital cost data from both academic and nonacademic sources to develop an understanding of recent cost trends.[15] These data are specific to Li-ion electric vehicle battery packs, rather than utility BESS. However, the EV market is a significant driver of battery costs and Li-ion technologies are often used for utility BESS. The consensus Nykvist and Nilsson report is that $\mathrm{Li}$-ion capital costs will continue to decline, settling asymptotically within the $\$ 150-\$ 300 / \mathrm{kWh}$ price range by 2025 .

\subsubsection{Aquion Battery}

Aqueous Hybrid Ion (AHI) batteries are one such technology that could satisfy the project requirements for affordability, safety, and low maintenance in battery choice. Aquion Energy's AHI batteries have a 15 to 20 year rated life with nearly zero maintenance requirements: no thermal management or active management is required, as in the case of lithium-ion and lead-acid batteries. They also feature a very high cycle life at $100 \% \mathrm{DoD}^{20}$, with very little capacity degradation over that time; have the ability to stand for long periods at partial state of charge; and are self-balancing.

Aquion's $1^{\text {st }}$-principles design requirements call for the use of abundant, nontoxic and relatively inert materials. The materials chosen for the battery are in great

\footnotetext{
${ }^{20}$ Depth of Discharge
} 
abundance around the world - magnesium oxide is used in the cathode, and Mn is the $12^{\text {th }}$ most common element in the earth's crust. The rarest element used in the batteries is sulfur, the $17^{\text {th }}$ most common. All of the materials are non-toxic and can be ingested in quantities a child might ingest without harm or lasting effects. The non-flammable, aqueous, salt-based electrolyte is commonly used as a laxative. Additionally, there is no risk of explosion in the case of a home fire, and no danger of catostrophic failure or toxic leakage in case of flooding. Aquion's batteries are the only batteries certified by the Cradle to Cradle organization, which examines a product and its effect on the environment from birth until end of life. To this point, the end of life disposal requirements are to recycle the polypropylene plastic housing and the metal hard goods, and dispose of the active battery materials as regular refuse due to their non-toxic and non-hazardous nature.

Aquion's battery was also designed intentionally for ease of fabrication. Conception to delivery of the first commercial battery took only 3 years. Funding and production for a full-scale factory took only two more years, which is a very short period for commercialization of a new battery technology. If manufacturing reaches the volume of Li-ion batteries, Aquion batteries will become more affordable, and the $\$ 200 / \mathrm{kWh}$ price point could very well be realized.

\subsubsection{Aquion Negatives}

One significant downside of these batteries in their low volumetric energy density, resulting in a large footprint per kWh. A $30.6 \mathrm{kWh}$ modular system, around $80 \%$ 
of what would be desired to bring the project vision to fruition, is $41.9^{\prime \prime} \mathrm{H}$ x 52.0 " W x 40.0" D; essentially a 3.5 foot high shipping pallet of batteries. This could prove troublesome in finding the space necessary within a customer's home; it is likely that we will need some flexibility from Aquion with regards to sizing - e.g. half as tall and twice as wide, etc.

Another downside is the power-to-energy ratio for this battery's chemistry. Even though significant advances have been made at Aquion, the most recent information from the company indicates that the continuous power rating for a new model M110-LS83 or M110-L083 $30.6 \mathrm{kWh}$ Aquion battery is only $8.1 \mathrm{~kW}$, with a peak power of $9.6 \mathrm{~kW} .{ }^{21}$ This can be contrasted with Tesla's newest specs for their Powerwall battery, where $32 \mathrm{kWh}$ results in a continuous power rating of $25 \mathrm{~kW}$ and a peak power of $35 \mathrm{~kW}$. This energy-to-power ratio for the Aquion battery places a limitation on the size of inverter and home that can be powered from these batteries. There is a slight positive to this, however: some of the power limitation arises from a relatively high internal impedance. This is advantageous under short circuit conditions, as the high impedance serves to limit fault current.

Finally, Aquion's ratings are very dependent on the rate of charge and discharge. For example, the $30.6 \mathrm{kWh}$ capacity of their newer model is given for a 20 -hour charge and discharge time; Were the battery to be discharged in 4 hours, even if it were charged for 20 hours, the capacity would decrease to $21.2 \mathrm{kWh}$. This also

\footnotetext{
${ }^{21}$ This is a $188 \%$ increase in continuous power and a $139 \%$ increase in peak power from March 2015 ratings for same form-factor model
} 
means DC round-trip efficiency can be as low as $80 \%$ if the battery is charged and discharged at 4-hour rates. The total range of round-trip efficiencies for the newest model is $80 \%-90 \%$.

\subsubsection{Batteries Used in Prototype}

For the initial prototype, sealed deep-cycle lead-acid batteries were chosen. Leadacid batteries were an affordable and proven technology, and they were appropriately sized for the initial prototype inquiry. Four $12 \mathrm{~V}, 21 \mathrm{Ah}$ batteries were used to achieve $84 \mathrm{Ah}$ of capacity, or around 2.5 hours with $3 \mathrm{~kW}$ of test loads running concurrently.

Most recently we have been using a single Aquion M100-L082 Battery Module, rated at $48 \mathrm{~V}_{D C}$ nominal, $4.3 \mathrm{~kW}$ continuous, and $25.5 \mathrm{kWh}$ at 20 hour discharge, $30^{\circ} \mathrm{C}$. This module came with a built in sensing board for metering, which was integrated into the prototype as discussed in Section 5.3.2.1. Lessons learned from this battery choice are detailed in Subsection 5.2.3.

\subsection{Inverter}

Several $\mathrm{kW}$-scale inverters were researched for the prototype. An issue identified during the search for an appropriate device is that off-the-shelf $\mathrm{kW}$-scale inverters are designed to be merely grid-tied; that is, they are designed to be used with a rooftop PV system and tied into the grid, but only in the most basic of ways. A simple grid-tied inverter is designed solely to interact with the grid by reverse- 
feeding power when the solar panels are producing more than the home or any installed batteries need. This results in an inverter with no $\mathrm{kW}$ or $\mathrm{kVAr}$ setpoints for battery charge or discharge, since this is not the intended use of the storage attached to an inverter. Furthermore, any battery storage attached to the inverter is intended to be discharged to the attached home when solar PV energy is not available - it is not intended to charge or discharge to the grid upon command in the way we intend.

This is changing somewhat; for instance, ABB's UNO-7.6 and UNO-8.6 kW-scale inverters show the ability to adjust power factor to \pm 0.8 , which gives some control over kVAr injection. Unfortunately, even an inverter specifically designed to be used with grid-tied storage, SMA's Sunny Island inverter (a consideration for the prototype), claims only to be "excellent for grid-tied battery back up" - and we were unable to gain information from the company as to whether the grid-tied storage could be used in any other fashion.

\subsubsection{Inverter Choice}

To demonstrate the desired functionality with the laboratory prototype, our primary design consideration was thus the ability to gain any level of control over a kW-scale, off-the-shelf inverter's internal setpoints. For this reason, a device from Outback Power was chosen for the prototype: a GTFX 3048, single-phase, $3 \mathrm{kVA}$ rated inverter. This choice was made because Outback manufactures an add-on device for their inverters called the AXS Port, which translates their inverters' internal 
set-points and metered data into a collection of registers available using the Modbus TCP data protocol. The use of the AXS port within our prototype is detailed in Section 5.3.2.2.

\subsubsection{Inverter Constraints}

One important constraint we identified in off-the-shelf kW-scale inverters - including the Outback chosen for the prototype - is that they are designed with two AC connections: one for a grid connection, and one for a customer load connection; these two being normally connected together in an electrically common AC bus. There is a normally-closed transfer relay on the AC bus, allowing for the grid and load sides of the inverter to be isolated in the case of a grid outage. The power electronics of the inverter are tied into this $\mathrm{AC}$ bus on the load side of the transfer relay, so as to provide battery power to the residence loads when the transfer relay is opened.

The constraint becomes a complication because in normal operation, the inverter desires to "pass-through" from the grid any current demanded by attached loads the current is passed across the transfer relay - while the inverter simultaneously manages PV and storage as necessary. In the GTFX 3048 inverter used in the lab, the transfer relay is only rated to $60 \mathrm{~A}$, which removes the inverter's ability to be used on any home with a greater than $50 \mathrm{~A}$ service connection ${ }^{22}$.

\footnotetext{
${ }^{22}$ if the single-phase inverter was to be used in a $120 / 240 \mathrm{~V}$ serviced home, it is possible to attach an additional inverter to provide the other hot leg with power
} 
Additionally, if this GTFX inverter were installed in a 50 A home, and the home were at rated current, then the $\mathrm{AC}$-side battery charging current would have to be limited to less than $10 \mathrm{~A}$ to protect the transfer relay, imposing an additional design and operational constraint. This transfer relay will likely result in a significant complication for future researchers.

\subsubsection{Constraints Involving Aquion Battery}

In the implementation of the Aquion battery, some limitations of the GTFX 3048 inverter were noted. The inverter has a voltage range of $42-68 V_{D C}$, and a maximum current input (battery discharge current) of $75 \mathrm{~A}_{D C}$ at rated power ( $\left.3 \mathrm{kVA}\right)$. The Aquion battery chosen has a voltage range of $30-59 \mathrm{~V}_{D C}$, and a maximum current output of $144 \mathrm{~A}_{D C}$ (assumed to be at rated power of $4.3 \mathrm{~kW}$ ).

These discrepancies are highlighted on the battery's datasheet, shown in Figure 5.1. The red lines show the limitations imposed by the inverter's current limits, and the orange lines the limitations imposed by voltage limits. 

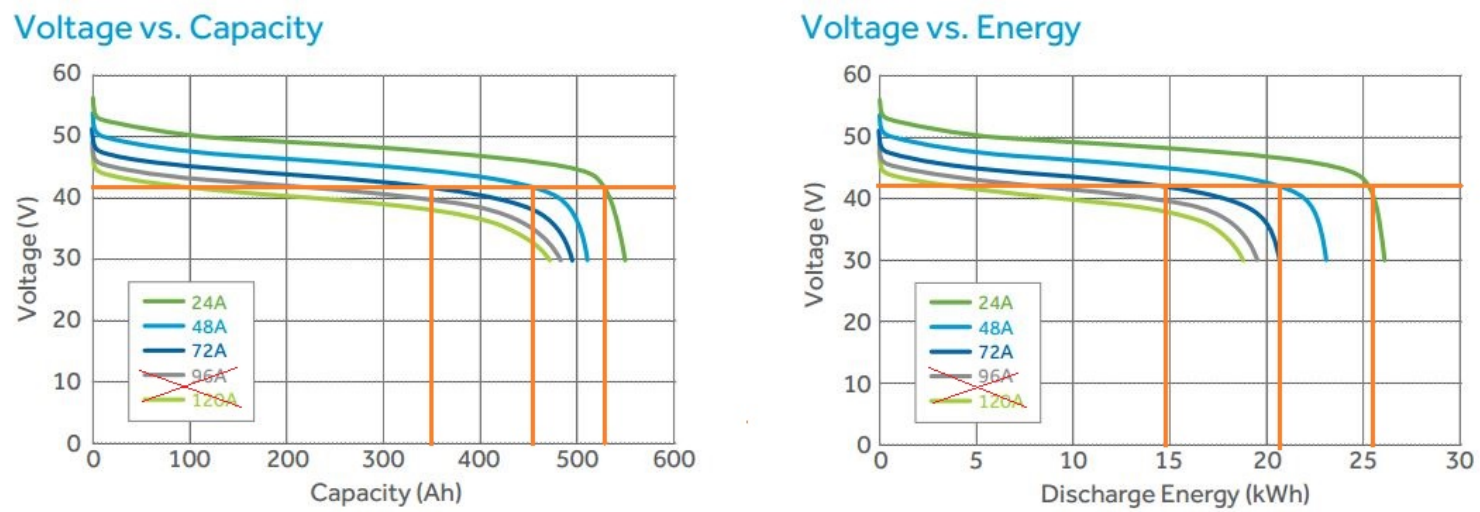

Current vs. Discharge Duration

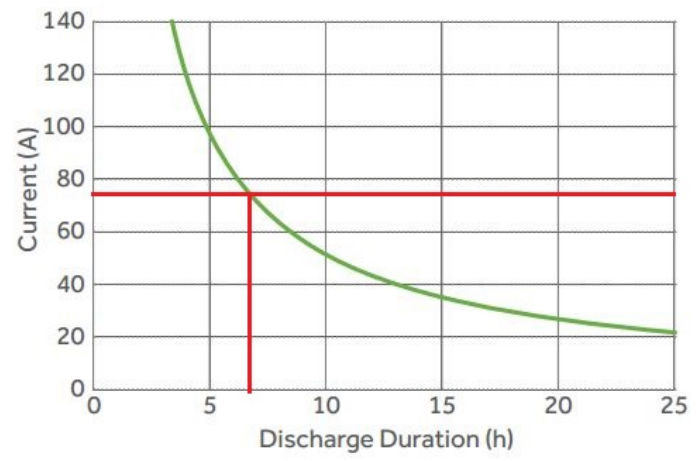

\section{Energy vs. Discharge Duration}

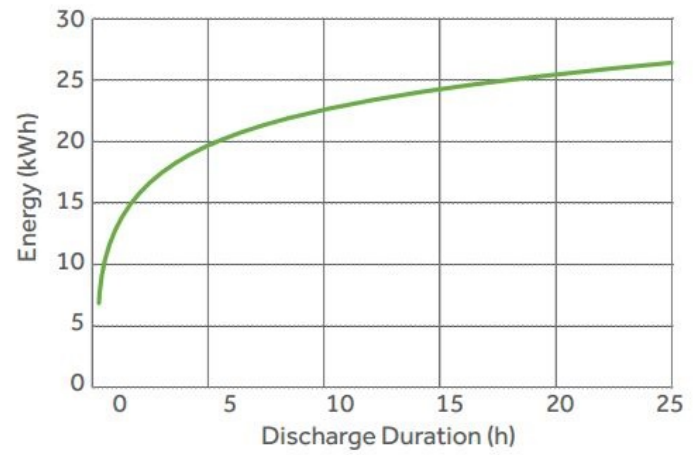

Figure 5.1: Battery performance data at continuous current: red lines show inverter current limits and orange lines show inverter voltage limits.

Source: Aquion M100-L082 Battery module datasheet; overlaid inverter data taken from Outback Energy GTFX3048 Inverter datasheet.

Since the inverter cannot sustain currents above $75 \mathrm{~A}_{D C}$, discharge times shorter than approximately 6.5 hours were not possible in the laboratory prototype. Furthermore, because of the inverter's lower voltage limit, the battery's Ah and kWh ratings are impacted as well. These energy impacts are most noticeable at faster discharge times, as we lose a substantial portion of the battery's capacity: at a $72 \mathrm{~A}_{D C}$ discharge, the voltage limitation removes over $5 \mathrm{kWh}$ of capacity from being accessed. Finally, with the battery rated at $4.3 \mathrm{~kW}$ and the inverter at $3 \mathrm{kVA}$, we were limited in the continuous power draw able to be placed upon the battery. 
The power rating mismatch may not be an issue if future researchers use a split-phase with higher ratings, but it will be desirable to engineer some ability to obtain access to the entirety of the battery's capacity - these batteries are not extremely energy dense to begin with. Aquion's newer batteries do have a tighter voltage range: now $40-57.6 \mathrm{~V}_{D C}$, so it may be easier to find an inverter capable of working within these bounds.

Additionally, the Aquion's battery used in the laboratory has a round-tripefficiency rating between $73 \%-90 \%$, while the inverter is reported to be generally $92 \%$ efficient but could be as low as $80 \%$ efficient. In the worst case, we could see

efficiencies as low as $\eta_{\text {total }_{\text {min }}}=\eta_{\text {inverter }_{\text {min }}} \times \eta_{\text {battery }}$ min $_{\text {in }}=80 \% \times 73 \%=58.4 \%$, and

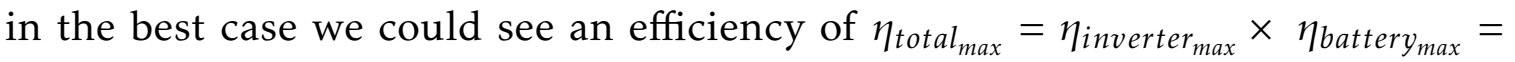
$92 \% \times 90 \%=82.8 \%$.

\subsection{Meter}

In section 4.1, the final system vision for the meter was discussed. There is no such meter on the market, nor anything even close to it. It is unheard of to combine $100 \mathrm{~A}$ relays and neutral access within the meter, built in communications and control capabilities, and the ability to interface through a single port with an attached inverter. Therefore, for our prototype we constructed an analog of the desired meter using a variety of disparate components. 


\subsubsection{Simulating Meter Communications and Control}

We sought to achieve remote control from the distributed standby generation department at PGE (GenOnSys) to the ResBESS prototype, to simulate the desired remote control and telemetry capabilities discussed in Section 4.1.4.

First, a secure communications link from the prototype to PGE was needed to ensure NERC CIP compliance: PGE cannot expose their internal network to external threats under current NERC CIP rules. ${ }^{23} \mathrm{~A}$ virtual private network (VPN) was briefly considered, but was discarded when we determined that the PSU Power Laboratory had an SEL real-time automation controller (RTAC) with the capability of assigning different MAC addresses to each communications port it possesses and separating communications internally with a firewall: this capability provided the necessary network isolation to satisfy security concerns. Additionally, because all data is either pulled by PGE from the RTAC or pushed by PGE to the RTAC, there is no data on PGE's system which is exposed to the RTAC.

PGE provided a CISCO router, which has the capability of taking communications from a device external to PGE as an ethernet IP input, and then broadcasting this device's data over a cellular 3G network for PGE to read. Likewise, PGE can broadcast commands over $3 \mathrm{G}$ and have them received by a device attached to the router. By assigning an ethernet port on the RTAC to recognize the static IP address of the router (10.1.2.254) and giving the RTAC the IP address of 10.1.2.96 (which

\footnotetext{
${ }^{23}$ An additional justification for classifying a deployed system of aggregated ResBESS units as a protected asset under NERC CIP rules is given in Appendix E.2.1
} 
the router was pre-configured to recognize), a static link was created between the RTAC and PGE.

The next step was to link the RTAC with the prototype, so that telemetry and metering data could be sent to PGE, and so that PGE could send control signals to the prototype - essentially making the RTAC the hub of the ResBESS prototype in a similar manner to the desired meter.

\subsubsection{Linking the RTAC to Prototype Devices}

The prototype has two main components to which the RTAC needed communication access: the battery and the inverter. Additionally, to simulate the desired meter functionality elucidated in Section 4.1, a meter was added to the prototype to gain accuracy in power metering; this also needed to be linked to the RTAC. The RTAC is limited to two RJ45 ethernet ports, and one was used to connect to PGE via the CISCO router; so the remaining RTAC RJ45 port was connected to an an 8-port Netgear network switch, allowing any additional devices with RJ45 ports to be connected to the switch - creating a local area network (LAN) - and thus giving the RTAC access via the LAN to any of these additional connected devices.

\subsubsection{Linking RTAC and Battery}

The Aquion battery used in the lab prototype has a built in sensing board, which collects data on voltages, currents, State of Charge (SOC), temperature, and more. We desired the DC Volts and DC Ampere data, as well as SOC, so that we could 
calculate DC Wh and DC power flow. The sensing board was connected via a CANBUS cable to a BMS-100 Battery Telemetry System (BTS) from Aquion, which compiles the data, and reports it over Modbus TCP via an RJ45 ethernet port.

The BTS was connected to the network switch previously mentioned, and configured with a Modbus TCP connection inside the RTAC using the BTS IP address of 192.168.254.52 at port 502 (the standard port for Modbus). Aquion provided documentation for the Modbus register list within the BTS; the registers desired were input registers in the 30,000 block (as opposed to the expected holding registers in the 40,000 block), but the documentation clearly listed addresses including offsets, and the registers were accessed easily. The RTAC automatically added 30,000 in this case, so to access register 30013 , only register 13 needed to be configured in the RTAC's input register list for the BTS.

\subsubsection{Linking RTAC and Inverter}

The inverter has a device called the AXS port, previously mentioned, which allows some control and telemetry over Modbus TCP via an RJ45 ethernet port. This port was used in past phases of the prototype to understand how to control the inverter, but was used within a different software package on a PC; we needed to reconfigure the connection for use within the RTAC.

Once again, the AXS port was connected to the network switch, and the RTAC was configured for a Modbus TCP connection to the AXS IP address of 192.168.0.64 at port 502. Outback also provided documentation for the Modbus register list 
within the AXS port, but their documentation expected understanding of an additional protocol layer: the SunSpec protocol. This protocol is a sort of common information model (CIM) similar to some of IEC standards, such as IEC 61850, where devices are identified by standardized digital ID's, and the device's data is lumped into a block consisting of many registers. The Outback documentation gave register addresses as an offset of the base address for their particular device block; e.g. register 268 was not 40268 as might be expected when using modbus, but was instead 268 removed from whatever address at which that block began.

Since the documentation was lacking, the desired registers for our purposes were found by starting at the base address of 40000 , where the SunSpec ID of 1850954613 was read (identifying the AXS port as a SunSpec device); subsequently reading the next register at address 40001, which gave a digital ID of 1 (identifying this "device" as the SunSpec protocol start block); and finally reading the register at address 40002 which gave a block length of 65 . The next block was then found 65 register addresses later, with a new device ID and block length; this block length was used to find the next block, and so on until all blocks had been mapped. The registers desired were all holding registers in the 40,000 block, but unlike when connecting to the BTS, this time the RTAC did not automatically add 40000; so to access register 40365 , register 40365 needed to be explicitly configured in the RTAC's input register list for the AXS port. 


\subsubsection{Linking RTAC and Meter}

Using the AXS port and the off-the-shelf inverter chosen, we have been able to achieve both on-off charging control of the battery, as well as a crude rate-ofdischarge control. The discharge rate can be adjusted by configuring a DC voltage set-point lower than the battery's actual voltage - the larger the difference between the actual and desired voltage, the greater the rate of discharge. To allow for PGE to set a desired power for discharge when using a similar inverter, future researchers will need to create a controller with a desired power reference signal (which the RTAC is currently able to receive from GenOnSys), a DC voltage set-point output (which the RTAC can send to the AXS port), and a measured discharged AC power from the inverter for feedback (which the inverter does not provide).

To obtain the discharged AC power measurements required for this control, PSU obtained a Schneider Electric ION 8600 meter on loan from PGE, which has real-time telemetry options. The meter was connected to the RTAC using a DB9 RS-485 physical layer for serial communications. The meter can use the Modbus RTU protocol over this connection to pass a variety of data to the RTAC, such as current, voltage, power (real, reactive and apparent), harmonics and more. Future researchers should be able to build on this initial connection to obtain any metered values desired. 


\subsubsection{Data Provided to PGE using RTAC to Prototype Device Connections}

Using the connections between the RTAC, the BTS and the AXS port, control and telemetry options for GenOnSys and PGE have been enabled. The data available to PGE as of December 16, 2015 are as seen in the following table:

\begin{tabular}{|r|l|l|l|l|l|}
\hline Register \# & Register Name & Contents & R/W & Units & Notes \\
\hline 0 & DC Current & 16 bit signed MSB & R & A & Scaling factor: $\mathrm{n}^{\star} 1000 / 32768$, negative sign is discharge \\
1 & Battery Voltage & 16 bit unsigned MSB & R & DC V & Scaling factor: $\mathrm{n}^{\star} 1000 / 65536$ \\
2 & Battery SOC & 16 bit unsigned MSB & $\mathrm{R}$ & $\%$ & Scaling factor. $\mathrm{n} / 10$ \\
\hline 3 & Battery Wh & 16 bit unsigned MSB & $\mathrm{R}$ & Wh & Scaling Factor. $\mathrm{n} / 100$, Pre-calculated on PSU side \\
4 & Charge Ctl & 16 bit unsigned MSB & R/W & N/A & Set to 1 to begin charging \\
5 & Discharge Ctl & 16 bit unsigned MSB & R/W & N/A & Set to 1 to begin discharging \\
\hline
\end{tabular}

Table 5.1: PGE to PSU Register List, Dec '15

The commands for charge and discharge control in this table are pushed to the RTAC by PGE, and run through the internal logic seen in the pseudo-code contained in Appendix $\mathrm{H}$ to accomplish their goal. This pseudo-code was implemented in our case using IEC 61131-3 structured text; a language similar to Pascal that the RTAC speaks.

As mentioned in Section 4.1.4.3, it may be desirable in future to limit the amount of data which is passed between utility and ResBESS units. Using the above table, the current system passes 96 bits to PGE, which would result in $300 \mathrm{kB}$ returned from all units in a scaled up system. Note that there is no information about system health in this data; which would increase the total amount of data returned to PGE. In this prototype configuration, the utility can broacast 32 bits for control, which would be $100 \mathrm{kB}$ at scale were every device commanded individually; however, if a mass-broadcast were used, and a command was encoded into a 32-bit word, 
this would reduce the sent command data to 64 bits: 32 bits for commands to be addressed to all units, or a group of units; and a 32 bit command word. 


\section{Conclusion \& Outcomes}

As mentioned at the beginning of this thesis, and reiterated several times throughout; the desired outcome of this thesis project is engineering knowledge that future researchers may use to inform design and construction of a field prototype or prototypes, eventually leading to the realization of an aggregated network of fully engineered and operational ResBESS units.

In Section 1, the problem was clearly stated, allowing future researchers to focus their work on solutions that are specific to the needs of an evolving electric utility. The proposed method of solving the problems posed by increasing amounts of renewable energy on the grid is but one method that may be employed - future work may involve a different set of benefits or desired outcomes from this thesis.

A brief review of existing or proposed solutions similar to this project - or more acurately, the lack thereof - was undertaken in Section 3, with the goal of identifying components and constraints faced by others in this field. With the specifics of equipment included in this document, this thesis can add to the growing body of knowledge for energy storage applications.

Section 4 went in depth into the future meter that would be needed to achieve the envisioned ResBESS solution, with consideration given to utility needs, ease of 
installation, prevailing industry standards, regulatory cyber-security requirements, and more. Additionally, a method of defining system states and the transitions between them was proposed, with the logic clearly explained for use in future. The needs of the utility were taken into account when defining the possible uses of a resource such as ResBESS, and the entirety of the section's information was combined into a set of technical specifications for an inverter and meter pair, intended for future researchers to refine for delivery to a manufacturing partner should the project move forward.

Finally, in Section 5, consideration was given to currently available off-the-shelf components needed to actualize the desired functionality proposed in prior sections. Where applicable, constraints and complications that arose with each component were noted so that these could be addressed or avoided by future researchers. Specifically, the components and methods used to simulate a meter that does not exist were outlined, such that another group of researchers would be able to build on this work.

\subsection{Future Work Underway}

The knowledge gained from this thesis project is already being applied by a team of undergraduate researchers, and though the field prototype they are designing is still in design and construction, as possible, this work will be shown here as a method of validating the thesis project. 
Recall that a method of communication and control devised for the single-phase lab prototype was detailed in Section 5.3.1. The current team has built upon this work using many of the same components and methods; a schematic of their proposed system configuration for the field prototype is shown in Figure 6.1, and described in the following sections. 


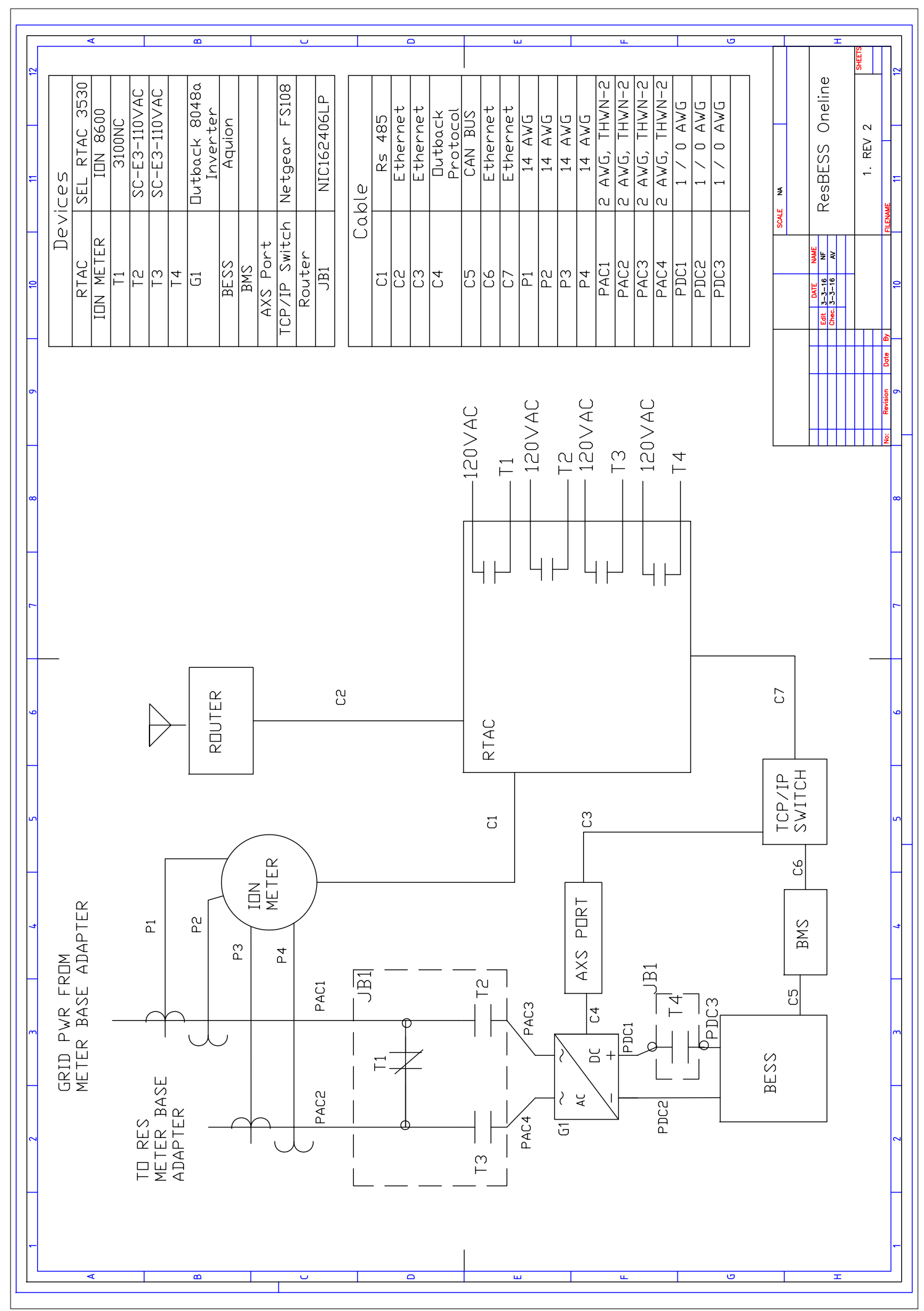

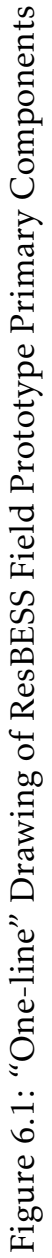




\subsubsection{Service Access}

A major consideration for the team is accessing electrical service to the home where the field prototype will be installed - this was not an issue in the laboratory installation because the "home" was virtual. Without the custom-built meter proposed, an alternate solution was devised: using a combination of access to the customer's service panel, and a custom meter-base-adapter, electrical service could be "broken" and routed through the prototype equipment. The wiring diagram showing this arrangement is seen in Figure 6.2, and the meter-base-adapter that enables it is diagrammed in Figure F.1 and Figure F.6, which are located in Appendix F.2. 


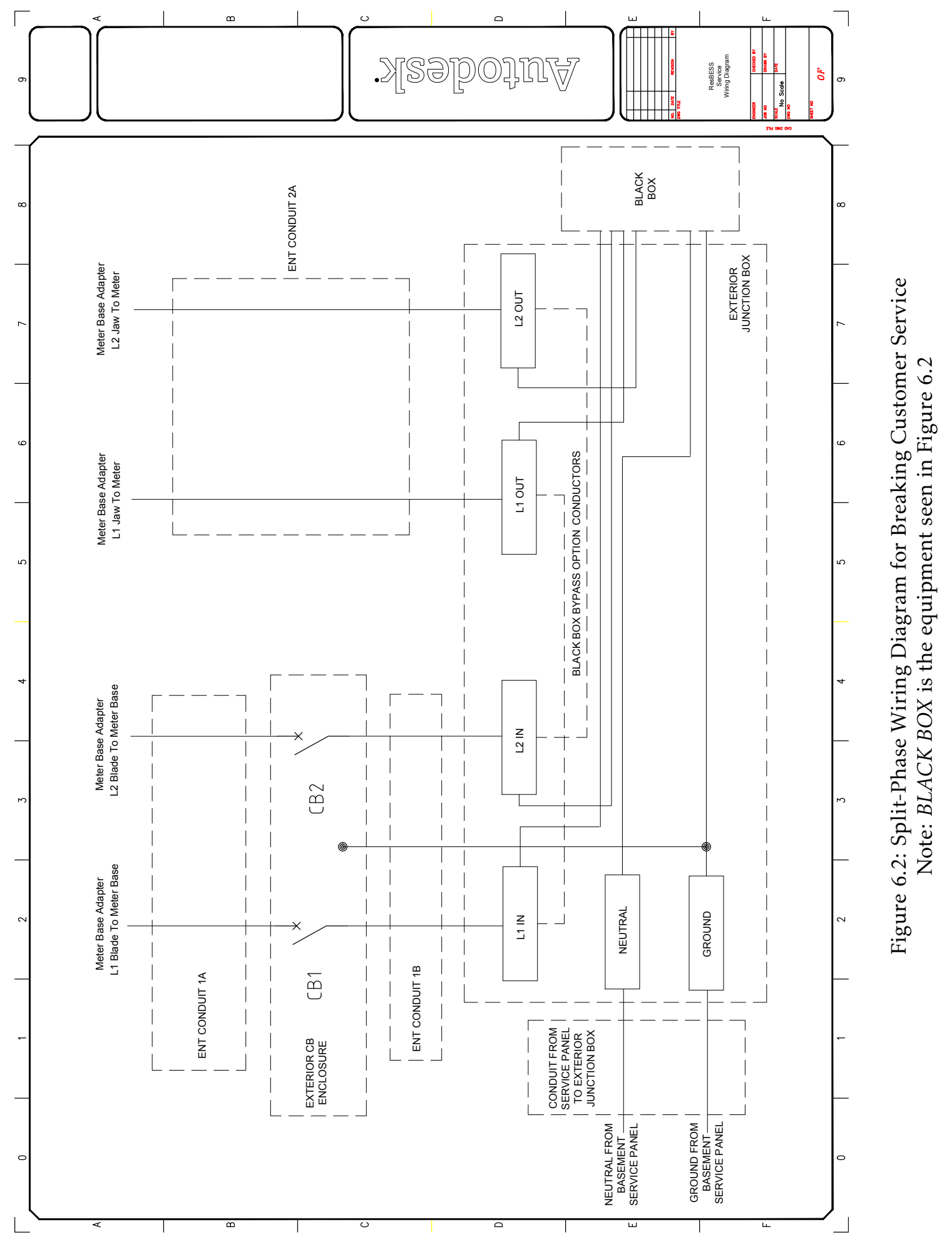




\subsubsection{Inverter Constraints Addressed}

Building on the success we had with an Outback inverter in the laboratory, the team has chosen another Outback inverter for the field installation: a GS-8048A split-phase, $8 \mathrm{kVA}$. Because this inverter also needs to be the central point for the grid/home/battery connection (the team had prior knowledge of this constraint from the information in Section 5.3.2.2) the home's electrical service is broken inside the meter-base for inverter access, with neutral and ground connections being made through a new conduit run to the customer's service panel. These conductors - L1, L2, N, and GND - are then passed to their inverter and other equipment. The two hot lines are electrically and physically pulled before they touch the customer's meter. Subsequently, they pass back, from their system to the customer's meter blades, the two hot lines alone: L1 and L2, for revenue metering by PGE. This allows the inverter the charge and discharge without the customers bill being affected, and also allows for the home be islanded in the case of an outage: an identified desire of the project in Section 2.2.2.

Recall, however, the limitation placed on the laboratory prototype by the inverter's internal relay ratings. This limitation is even more pronounced in the inverter chosen for the field prototype: a maxiumum of $50 \mathrm{~A}$ can pass through the inverter per leg of service (as compared to $65 \mathrm{~A}$ in the lab). To keep the customer's existing 100 A electrical service rating, a "by-pass" was devised by the research team. By placing 65 A normally-open relays (labeled T2 and T3 in Figure 6.1) on 
the hot lines on either side of the inverter, we gain the ability to remove the inverter from the system if it is experiencing an over-current condition (and for it to fail out of the system if power is lost). However, this only solves the possibility of damaging equipment; since the inverter is the central point for the grid/home/battery connection, opening the 65 A relay pair will also take away the "pass-through" capabilities of the inverter and will subsequently eliminate electrical service to the home.

To alleviate this issue, a 100 A normally-closed relay (labeled T1 in Figure 6.1) is placed across the two hot-line connections to the inverter - allowing for complete bypass of the inverter. When the $100 \mathrm{~A}$ relay is closed, and the two $65 \mathrm{~A}$ relays are opened, the home is electrically returned to a state where none of the equipment is installed. Note that by choosing the $100 \mathrm{~A}$ relay to be $\mathrm{NC}$ and the $65 \mathrm{~A}$ relays to be NO, this bypass is the failure state upon complete loss of power. These additional relays change the conceptual switching logic outlined in Section 4.2.3 and Appendix G.5; this work is yet to be done by the team.

\subsubsection{New Constraints}

A major consideration in the design of the new switching logic is preventing the design from "ratcheting" back and forth between bypass and inverter configurations - there will need to be some delay for the system to reach a steady-state condition before either moving to bypass, or exiting bypass. Secondarily, to keep the inverter's grid and load connections from seeing matching voltages, the team will need to ensure break-before-make behavior for the relays: the inverter must lose grid 
connection a brief moment before the bypass is engaged, and the bypass must break for a brief moment before the inverter is re-entered into the system.

\subsubsection{RTAC Control Loop}

The team has done additional work recently to advance and refine the control capabilities of the laboratory system using the RTAC's built-in controller capabilities; however, full documentation from this work has not been completed. This controller is intended to be ported to the field-prototype inverter. A very rough draft of the results of this work are presented here.

Some preliminary plots of the data collected when testing control algorithms are shown on the following pages. Using the rough discharge control methods achieved in the thesis work, and the metering capabilites provided in Section 5.3.2.3, the team has built a method of setting the discharge rate of the single-phase inverter to a specific output power, as intended. This will allow PGE operators and future researchers the ability to test the field installation's ability to meet many of the use-case technical needs outlined in Section 4.4.1.

In Figure 6.3, it appears that the inverter responds to a desired power discharge set-point within 2 seconds, and fully ramps within around 30 seconds. More detailed analysis is needed once this control algorithm is ported to the field installation, clearly; but this initially suggests that the off-the-shelf inverter in the laboratory is too slow to be a frequency-regulating device (fully-ramped within 4 
seconds), but fast enough to be considered a spinning-reserve (ramped within 10 minutes).

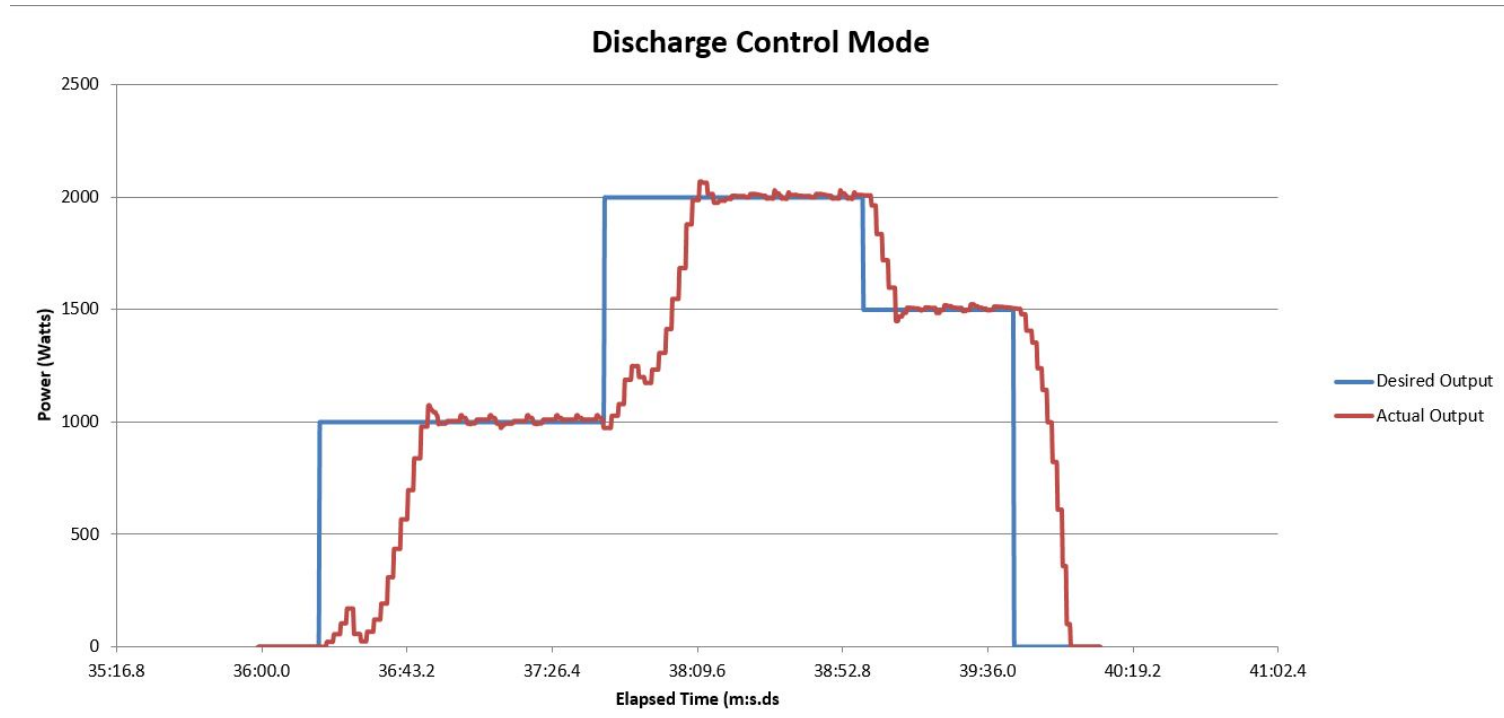

Figure 6.3: Desired-Power Discharge Step-Response Control-Loop Test Data

To enable the ability to shift load to off peak times, as mentioned in Section 4.3.1.3, the team has created a control algorithm that matches the output of the inverter to the loads seen by the inverter. In Figure 6.4, we see that the inverter was first commanded to "shed" laboratory test loads, and that when a load appears at 36:08, the inverter begins to respond within 2 seconds to accomplish this goal. The load is fully compensated for by the inverter within 28 seconds of its appearance. This is not extremely rapid, but this initially suggests that the system could be pre-scheduled for peak shaving. Further testing would be desired to see what occurs when the command to peak-shave is given while loads are already running, which is a more realistic scenario. 


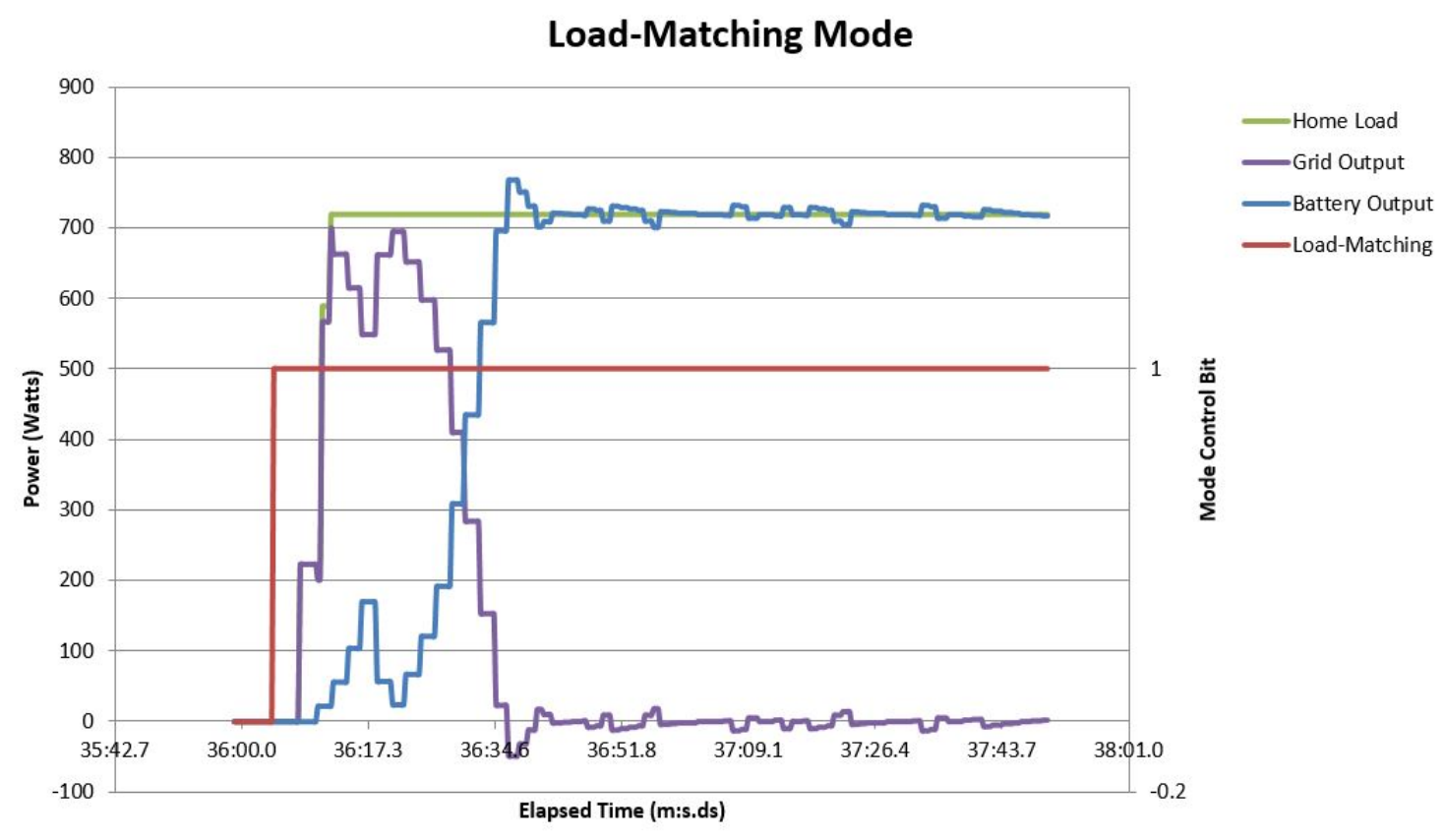

Figure 6.4: Load-Matching (Peak Shaving) Control-Loop Test Data 


\section{Bibliography}

[1] G. Wu and K. Chubachi. Improvement of imbalance and FRT capacity in distribution voltage deteriorated by integration of renewable power generations by use of STATCOM technology. In 2012 IEEE International Conference on Power System Technology (POWERCON), pages 1-6, October 2012. doi: 10.1109/PowerCon.2012.6401381.

[2] M. Huotari, T. Tengnér, and N. Johansson. Control of Energy Storage units to limit active power ramp-rates of renewable energy sources using non-ideal generation forecasts. In North American Power Symposium (NAPS), 2015, pages 1-6, October 2015. doi: 10.1109/NAPS.2015.7335126.

[3] Database of State Incentives for Renewables \& Efficiency. Renewable portfolio standard policies. http://ncsolarcen-prod.s3.amazonaws.com/wp-content/ uploads/2015/11/Renewable-Portfolio-Standards.pdf. Accessed: April 18, 2016.

[4] Direct Testimony to OPUC. Ue-283 general rate case 2015 test year - exhibit 400. https://www.portlandgeneral.com/our-company/regulatory-documents/ filings/ue-283-general-rate-case-2015-test-year, . Accessed: April 7, 2016. 
[5] Debra Lew, Greg Brinkman, E. Ibanez, B. M. Hodge, and J. King. The western wind and solar integration study phase 2. Contract, 303:275-3000, 2013. URL https://www.researchgate.net/profile/Eduardo_Ibanez/publication/ 273000140_The_Western_Wind_and_Solar_Integration_Study_Phase_2/ links/54f485ab0cf2ba6150634872.pdf.

[6] U.S. Energy Information Administration. Today in energy, march 12016. https://www.eia.gov/todayinenergy/detail.cfm?id=25172\#. Accessed: April $18,2016$.

[7] Direct Testimony to OPUC. Ue-294 general rate case 2016 test year - exhibit 800. https://www.portlandgeneral.com/our-company/regulatory-documents/ filings/ue-294-general-rate-case-2016-test-year, . Accessed: April 7, 2016.

[8] J. Schellenberg M. Sullivan and M. Blundell. Updated Value of Service Reliability Estimates for Electric Utility Customers in the United States. http: //emp.lbl.gov/sites/all/files/value-of-service-reliability-final.pdf.pdf. Accessed: April 7, 2016.

[9] E. Manasseh, S. Ohno, T. Yamamoto, and A. Mvuma. Autonomous demandside optimization with load uncertainty. In 2014 International Conference on Electronics, Information and Communications (ICEIC), pages 1-2, January 2014. doi: 10.1109/ELINFOCOM.2014.6914355. 
[10] R. S. Weissbach and J. R. King. Estimating Energy Costs Using a Markov Model for a Midwest Off-Grid Residence. In 2013 IEEE Green Technologies Conference, pages 430-434, April 2013. doi: 10.1109/GreenTech.2013.72.

[11] R. S. Weissbach, R. E. Teodorescu, and J. R. Sonnenmeier. Comparison of Time-Based Probability Methods for Estimating Energy Storage Requirements for an Off-Grid Residence. In IEEE Energy 2030 Conference, 2008. ENERGY 2008, pages 1-4, November 2008. doi: 10.1109/ENERGY.2008.4781019.

[12] H. S. Krishnamoorthy, D. Rana, and P. N. Enjeti. A new wind turbine generator / battery energy storage utility interface converter topology with mediumfrequency transformer. In 2013 Twenty-Eighth Annual IEEE Applied Power Electronics Conference and Exposition (APEC), pages 2218-2224, March 2013. doi: 10.1109/APEC.2013.6520603.

[13] S. Essakiappan, M. Manjrekar, J. Enslin, J. Ramos-Ruiz, P. Enjeti, and P. Garg. A utility scale battery energy storage system for intermittency mitigation in multilevel medium voltage photovoltaic system. In 2015 IEEE Energy Conversion Congress and Exposition (ECCE), pages 54-61, September 2015. doi: 10.1109/ECCE.2015.7309669.

[14] Y. Wen, C. Guo, H. Pandžić, and D. S. Kirschen. Enhanced SecurityConstrained Unit Commitment With Emerging Utility-Scale Energy Storage. 
IEEE Transactions on Power Systems, 31(1):652-662, January 2016. ISSN 08858950. doi: 10.1109/TPWRS.2015.2407054.

[15] Björn Nykvist and Måns Nilsson. Rapidly falling costs of battery packs for electric vehicles. Nature Climate Change, 5(4):329-332, April 2015. ISSN 1758678X. doi: 10.1038/nclimate2564. URL http://www.nature.com/nclimate/ journal/v5/n4/full/nclimate2564.html.

[16] Navigant Research. Community, Residential, and Commercial Energy Storage. http://www.navigantresearch.com/research/ community-residential-and-commercial-energy-storage. Accessed: April 28, 2016.

[17] G. Fitzgerald, J. Mandel, J. Morris, and H. Touati. The Economics of Battery Energy Storage: How multi-use, customer-sited batteries deliver the most services and value to customers and the grid. http://www.rmi.org/Content/ Files/RMI-TheEconomicsOfBatteryEnergyStorage-FullReport-FINAL.pdf. Accessed: April 28, 2016.

[18] J. Jeff St. How Austin Energy Aims to Reach Solar-Plus-Storage Integration at 14 Cents per Kilowatt-Hour. http://www.greentechmedia.com/articles/read/ How-Austin-Energy-Aims-to-Reach-Solar-Plus-Storage-Integration-at-14-Cents. Accessed: April 18, 2016. 
[19] Consolidated Edison. REV Demonstration Project Outline: Clean Virtual Power Plant. http://www.capitalnewyork.com/sites/default/files/ CONEDDEMO3.pdf. Accessed: April 8, 2016.

[20] Heschong Mahone Group, Inc. Technology Evaluation Of Programmable Communicating Thermostats With Radio Broadcast Data System Communications. http://www.energy.ca.gov/2012publications/CEC-500-2012-077/ CEC-500-2012-077.pdf. Accessed: April 25, 2016.

[21] M. Kgwadi and T. Kunz. Securing rds broadcast messages for smart grid applications. http://citeseerx.ist.psu.edu/viewdoc/download?doi=10.1.1.216. 5096\&rep=rep1\&type=pdf. Accessed: April 25, 2016.

[22] IEEE guide for monitoring, information exchange, and control of distributed resources interconnected with electric power systems. IEEE Std 1547.3-2007, pages 1-158, 2007. doi: 10.1109/IEEESTD.2007.4432408.

[23] Itron, Inc. Certicom to Provide Itron with Innovative AMI Security Capabilities. https://www.itron.com/na/newsAndEvents/Pages/ Certicom-to-Provide-Itron-with-Innovative-AMI-Security-Capabilities. aspx. Accessed: April 25, 2016.

[24] IEEE standard for interconnecting distributed resources with electric power systems. IEEE Std 1547-2003, pages 1-28, July 2003. doi: 10.1109/IEEESTD. 2003.94285. 
[25] NERC. CIP-002-3: Cyber Security - BES Cyber System Categorization. http://www.nerc.com/_layouts/PrintStandard.aspx?standardnumber= CIP-002-3.1\&title=Cyber $\% 20$ Security $\% 20 \%$ E2\%80\%94\%20BES $\% 20$ Cyber $\%$ 20System\%20Categorization\&jurisdiction=null, . Accessed: March 4, 2015.

[26] NERC. CIP-002-5: Cyber Security - BES Cyber System Categorization. http://www.nerc.com/_layouts/PrintStandard.aspx?standardnumber= CIP-002-5.1\&title=Cyber $\% 20$ Security $\% 20 \%$ E2\%80\%94\%20BES $\% 20$ Cyber $\%$ 20System\%20Categorization\&jurisdiction=null, . Accessed: March 17, 2015.

[27] NERC. Lesson Learned, CIP Version 5 Transition Program. http://www.nerc. com/pa/Stand/CIP00251RD/CIP-002-5.1\%20Impact\%20Rating\%20of\% 20Generation\%20Resource $\% 20$ Shared\%20BES\%20Cyber\%20Systems.pdf, . Accessed: March 9, 2015. 


\section{Appendix A: Supplementary Analysis}

Some supplementary analysis which did not seem relevant to the thesis project, yet was completed by the researcher over the course of the project for personal interest, is presented here in hopes that it can be useful in future.

First, a high-level cost analysis is presented for a scaled ResBESS system; and a maintenance program is also proposed, with a failure-mode analysis to justify the program.

\section{A.1 Costs}

\section{Cost Assumptions:}

- Costs are reduced in some cases by working with vendors to increase volume development

- Initial engineering and associated investment is completed, system is at 7200 units out of final 25,000 (year 2021).

- Battery at lowest (year 2025) is $\$ 190 / \mathrm{kWh}$, at low (year 2021) is $\$ 215 / \mathrm{kWh}$, and at high (year 2016) is $\$ 300 / \mathrm{kWh}$

- Battery is $52.8 \mathrm{kWh}$ 
- Inverter at lowest (year 2025) is $\$ 250 / \mathrm{kW}$, at low (year 2021) is $\$ 290 / \mathrm{kW}$, and at high (no year, for risk assessment) is $\$ 350 / \mathrm{kW}$

- Inverter is $8 \mathrm{~kW}$

- High profit margin for meter manufacturer assumed to get buy-in for custom design partnership; current meters approx $\$ 180$

- Meter at lowest (no year, for risk assessment) is $\$ 300$, at low (year 2021) is $\$ 350 / \mathrm{kW}$, and at high (no year, for risk assessment) is $\$ 400 / \mathrm{kW}$

- Install components include custom weatherproof container for batteries/inverter; very rough estimates

- Install labor includes 14 man-hours $\$ 60 / \mathrm{hr}$, plus $\$ 100$ per install for amortized cost of $\$ 100 \mathrm{k}$ purchase of machinery needed for install. Final number is estimate from industry professional.

- Office overhead includes Project Manager for install teams, sales, and support of ResBESS program customers.

Unless otherwise specified, the ranges of costs for components (lowest to high) were pure estimates to account for the risk of labor costs rising, parts costing more, etc.

The following page shows tables with all assumptions made and costs summed. 


\begin{tabular}{|c|c|c|c|c|c|c|c|c|c|}
\hline & Battery & Inverter & Me & & Inst & ients & Tot & st/unit & Capital cost of $25 \mathrm{k}$ units \\
\hline Lowest & $\$ 10,032$ & $\$ 2,000$ & $\$$ & 300 & $\$$ & 550 & $\$$ & 12,882 & $322,050,000$ \\
\hline Low & $\$ 11,352$ & $\$ 2,320$ & $\$$ & 350 & $\$$ & 550 & $\$$ & 14,572 & $364,300,000$ \\
\hline High & $\$ 15,840$ & $\$ 2,800$ & $\$$ & 400 & $\$$ & 800 & $\$$ & 19,840 & $496,000,000$ \\
\hline
\end{tabular}

Table A.1: Capital Cost Estimates

\begin{tabular}{|c|c|c|c|c|c|c|c|c|c|c|}
\hline & Battery & $\operatorname{lnv}$ & & Meter & Ins & II labor & omponents & Office Overhead & Total cost/unit & ost of $25 \mathrm{k}$ units \\
\hline Lowest & $\$ 10,032$ & $\$$ & 2,000 & $\$ 300$ & $\$$ & 1,600 & 550 & 125 & 14,607 & 5,000 \\
\hline Low & $\$ 11,352$ & $\$$ & 2,320 & $\$ 350$ & $\$$ & 1,600 & 550 & 125 & 16,297 & $407,425,000$ \\
\hline High & $\$ 15,840$ & $\$$ & 2,800 & $\$ 400$ & $\$$ & 2,000 & 800 & 175 & 22,015 & $550,375,000$ \\
\hline
\end{tabular}

Table A.2: System Cost Estimates 


\section{A.2 Maintenance}

Maintenance is expected to be lower for this resource than other energy storage solutions, due to the Aquion battery having no need for a Battery Managment System or needing scheduled maintenance. The metering and telemetry system will be designed to report issues or faults back to the control center with each update (or on command), and even failure to respond or check in will trigger an exception in the database of ResBESS telemetry.

Based on the above, we would recommend a corrective-based maintenance program for the battery, and a condition-based program for the inverter and meter/control components. A draft failure mode and effects analysis supporting this recommendation is shown below. 


\begin{tabular}{|c|c|c|c|c|c|c|c|c|c|}
\hline Item / Function & $\begin{array}{c}\text { Potential } \\
\text { Failure Mode[s] }\end{array}$ & $\begin{array}{l}\text { Potential } \\
\text { Effect(s) } \\
\text { of Failure }\end{array}$ & $\left|\begin{array}{c}s \\
e \\
v\end{array}\right|$ & $\begin{array}{l}\text { Potential } \\
\text { Cause[s]! } \\
\text { Mechanism[s] } \\
\text { of Failure }\end{array}$ & $\begin{array}{l}P \\
r \\
0 \\
b\end{array}$ & $\begin{array}{c}\text { Current Design } \\
\text { Controls }\end{array}$ & $\begin{array}{l}\mathrm{D} \\
\mathrm{e} \\
\mathrm{t}\end{array}$ & $\begin{array}{l}R \\
P \\
N\end{array}$ & $\begin{array}{c}\text { Recommended } \\
\text { Action[s] }\end{array}$ \\
\hline \multicolumn{10}{|l|}{$\overline{\text { Battery }}$} \\
\hline Charge & $\begin{array}{l}\text { Will not hold } \\
\text { charge }\end{array}$ & $\begin{array}{l}\text { No backup } \\
\text { available to } \\
\text { customer, utility } \\
\text { loses resource }\end{array}$ & 8 & $\begin{array}{l}\text { Failure of battery } \\
\text { chemistry }\end{array}$ & 2 & & 2 & 32 & \\
\hline Fault & $\begin{array}{l}\text { Short between } \\
\text { terminals or } \\
\text { ground }\end{array}$ & $\begin{array}{l}\text { Rise in battery } \\
\text { temperature, } \\
\text { inverter'meter } \\
\text { damage }\end{array}$ & 10 & $\begin{array}{l}\text { Tampering with } \\
\text { battery }\end{array}$ & 4 & $\begin{array}{l}\text { Sealed battery } \\
\text { container }\end{array}$ & & 40 & \\
\hline \multicolumn{10}{|l|}{ Inverter } \\
\hline Fault & $\begin{array}{l}\text { Short between } \\
\text { terminals or } \\
\text { ground }\end{array}$ & $\begin{array}{l}\text { Inverter ' meter ' } \\
\text { battery damage, or } \\
\text { residence } \\
\text { damage. } \\
\text { Utility loses } \\
\text { resource }\end{array}$ & 10 & $\begin{array}{l}\text { Tampering with } \\
\text { inverter }\end{array}$ & 4 & $\begin{array}{l}\text { Sealed inverter } \\
\text { design }\end{array}$ & 2 & 80 & $\begin{array}{l}\text { Condition-based } \\
\text { maintenance }\end{array}$ \\
\hline Fault & $\begin{array}{l}\text { Short within } \\
\text { inverter }\end{array}$ & $\begin{array}{l}\text { Inverter I meter ' } \\
\text { battery damage, or } \\
\text { residence } \\
\text { damage. } \\
\text { Utility loses } \\
\text { resource }\end{array}$ & 10 & $\begin{array}{l}\text { Manufacturing } \\
\text { defect, aging of } \\
\text { inverter }\end{array}$ & 4 & $\begin{array}{l}\text { Detection for } \\
\text { internal faults }\end{array}$ & 2 & 80 & $\begin{array}{l}\text { Condition-based } \\
\text { maintenance }\end{array}$ \\
\hline \multicolumn{10}{|l|}{ Meter } \\
\hline $\begin{array}{l}\text { Failure to control } \\
\text { inverter }\end{array}$ & $\begin{array}{l}\text { Reciever failure: } \\
\text { Inverter shuts } \\
\text { down, does not } \\
\text { respond, or ramps } \\
\text { to limit }\end{array}$ & $\begin{array}{l}\text { Utility loses } \\
\text { resource; possible } \\
\text { equipment } \\
\text { damage }\end{array}$ & 6 & $\begin{array}{l}\text { Manufacturing } \\
\text { defect, aging of } \\
\text { meter, weather }\end{array}$ & 2 & $\begin{array}{l}\text { Detection in } \\
\text { master control } \\
\text { system, } \\
\text { weatherproofing }\end{array}$ & 4 & 48 & $\begin{array}{l}\text { Condition-based } \\
\text { maintenance }\end{array}$ \\
\hline $\begin{array}{l}\text { Failure to } \\
\text { communicate with } \\
\text { utility }\end{array}$ & $\begin{array}{l}\text { RF Mesh failure, } \\
\text { individual meter } \\
\text { transmit failure }\end{array}$ & $\begin{array}{l}\text { Utility loses } \\
\text { resource; possible } \\
\text { equipment } \\
\text { damage, unknown }\end{array}$ & 9 & $\begin{array}{l}\text { Manufacturing } \\
\text { defect, aging of } \\
\text { meter, weather }\end{array}$ & 5 & $\begin{array}{l}\text { Detection in } \\
\text { master control } \\
\text { system, } \\
\text { weatherproofing }\end{array}$ & 1 & 45 & \\
\hline
\end{tabular}

Table A.3: Draft FMEA for ResBESS 


\section{Appendix B: Summary Table}

\begin{tabular}{|l|l|}
\hline Requirements & Notes \\
\hline \hline $\begin{array}{l}\text { 240 Connection for } \\
\text { System }\end{array}$ & $\begin{array}{l}\text { Avoids 5\% losses in last 100 feet to house, provides } \\
\text { ability to give customer backup power. }\end{array}$ \\
\hline Neutral Access & $\begin{array}{l}\text { Through meter base if possible, otherwise least cost } \\
\text { upon installation. Necessary to provide inverter the } \\
\text { ability to supply 120V lines in backup. }\end{array}$ \\
\hline $\begin{array}{l}\text { Processing Power } \\
\text { within System }\end{array}$ & $\begin{array}{l}\text { Robust within either in meter or inverter (or both), al- } \\
\text { lowing distributed intelligence and localized control. }\end{array}$ \\
\hline $\begin{array}{l}\text { Data Antennae } \\
\text { within System }\end{array}$ & $\begin{array}{l}\text { Either in meter or inverter (or both), allowing receipt of } \\
\text { mass broadcast commands and telemetry returned to } \\
\text { utility. }\end{array}$ \\
\hline $\begin{array}{l}\text { Bi-directional Power } \\
\text { Connection }\end{array}$ & $\begin{array}{l}\text { Within meter, ability to send/receive power from in- } \\
\text { verter; within inverter, elimination of pass-through re- } \\
\text { quirement and transfer relay limitations. }\end{array}$ \\
\hline $\begin{array}{l}\text { System Disconnect } \\
\text { Switches }\end{array}$ & $\begin{array}{l}\text { Fully-rated (100A min) disconnect switches on both hot } \\
\text { lines and on bi-directional inverter connection, allow- } \\
\text { ing for ability to configure system connections. }\end{array}$ \\
\hline $\begin{array}{l}\text { Meter/System } \\
\text { Backup Power }\end{array}$ & $\begin{array}{l}\text { Ability for meter and system intellegence to continue } \\
\text { running in the event of grid outage (power from inverter } \\
\text { or internal UPS). }\end{array}$ \\
\hline $\begin{array}{l}\text { IEEE 1547 Compli- } \\
\text { ance }\end{array}$ & $\begin{array}{l}\text { Design system for IEEE 1547 conformity, with ability } \\
\text { to override as desired by utility. }\end{array}$ \\
\hline
\end{tabular}

Table B.1: High-level Summary Table of System Requirements and Design Considerations

Table continued on next page 


\begin{tabular}{|c|c|}
\hline Requirements & Notes \\
\hline $\begin{array}{l}\text { IEEE } 1547 \text { Compli- } \\
\text { ance }\end{array}$ & $\begin{array}{l}\text { Design system for IEEE } 1547 \text { conformity, with ability } \\
\text { to override as desired by utility. }\end{array}$ \\
\hline $\begin{array}{l}\text { NERC CIP Compli- } \\
\text { ance }\end{array}$ & $\begin{array}{l}\text { Design system for NERC CIP conformity, with ability } \\
\text { to override as desired by utility: required at } 300 \mathrm{MW} \text {, } \\
\text { otherwise may be optional. }\end{array}$ \\
\hline $\begin{array}{l}\text { Radio } \text { Broadcast } \\
\text { Method }\end{array}$ & Use IEC 62106 for secured mass broadcast to all devices. \\
\hline $\begin{array}{ll}\text { Return } & \text { Telemetry } \\
\text { Method } & \end{array}$ & $\begin{array}{l}\text { Use RF mesh (IPv6?) for returned data, compliant to } \\
\text { IEC } 61850-7-420 \text { and IEC } 61970 .\end{array}$ \\
\hline Status Signals & $\begin{array}{l}\text { Allow system to automatically configure configurations } \\
\text { based on primarily local device and grid health; allow } \\
\text { global monitoring and control of local status signals by } \\
\text { utility if desired. }\end{array}$ \\
\hline $\begin{array}{l}\text { System } \quad \text { Response } \\
\text { Speed }\end{array}$ & $\begin{array}{l}\text { Design system for fast and accurate response to satisfy } \\
\text { frequency response requirements: accomplishing this } \\
\text { will allow system to satisfy other contingency reserve } \\
\text { needs and renewable generation following. }\end{array}$ \\
\hline Full VAr Control & $\begin{array}{l}\text { Allow complete control over power factor so system can } \\
\text { supply or consume within four quadrants of power }\end{array}$ \\
\hline $\begin{array}{l}\text { Match Battery and } \\
\text { Inverter Ratings }\end{array}$ & $\begin{array}{l}\text { Ensure all ratings for inverter and battery are matched } \\
\text { to provide full access to capacity of battery }\end{array}$ \\
\hline Full Inverter Control & $\begin{array}{l}\text { Design for full ability to control all setpoints of inverter, } \\
\text { utility assuming all risk of mis-operation. }\end{array}$ \\
\hline $\begin{array}{l}\text { Full System Meter- } \\
\text { ing }\end{array}$ & $\begin{array}{l}\text { Design ability to independently meter all lines within } \\
\text { system to ensure full observability in any system switch } \\
\text { configuration. }\end{array}$ \\
\hline
\end{tabular}

Table B.2: High-level Summary Table of System Requirements and Design Considerations, cont. 
Appendix C: Technical Specifications

\section{C.1 Meter Specifications}

1. The meter shall connect to a standard 200 A meter socket.

2. The meter shall have two $240 \mathrm{~V}$ power ports, rated at $200 \mathrm{~A}$ continuous, that connect to a meter socket.

3. The meter shall have one three conductor, four wire (L1, L2, N, GND), $240 \mathrm{~V}$ power port, rated at 30 A continuous, that connects at the meter body.

4. The port on the meter body shall accommodate both power (L1, L2, N) and a data communication point between the meter and inverter via a single socket.

5. The meter shall have a port rated at 30 amps continuous which is located in a manner allowing connection to the neutral line $(\mathrm{N})$ in the meter base, and passes through the meter to the inverter.

6. The meter shall be able to calculate power at each of the three power ports (residence, utility, inverter).

7. The meter shall include remote disconnect switches at two of the power ports (residence, utility). 
8. The meter shall have the capability, likely in conjunction with the inverter, to determine the state of voltage and frequency at the point of common connection.

9. The meter shall coordinate with the inverter to perform a sync-check relay function for the purpose of synchronizing the ResBESS at the point of common connection.

10. The meter shall be capable of actuating the disconnect switch at the inverter, unless that switch has been manually locked.

11. The meter shall maintain its internal power supply during all use cases.

12. The meter shall be capable of receiving broadcast data transmissions from the utility.

13. The meter shall be capable of reporting and data measurements and system status on a regular basis, as often as once every 5 minutes.

14. The meter shall have sufficient memory to buffer measurement and status data in anticipation of the regular reporting period.

15. Each meter shall be addressable using a unique numerical identifier.

16. The rated temperature range should be $0^{\circ} \mathrm{C}$ to $50^{\circ} \mathrm{C}$.

17. The receive communications method should be a broadcast technique, such as an open FM broadcast subcarrier channel. 
18. The transmit communication method should be a standard technique commonly used by utilities, such as cellular, TCP/IP, BPL, etc.

19. The transmit and receive communications protocol should be a standard protocol commonly used by utilities.

20. The data communications channel between the meter and the inverter should use a standard protocol 


\section{C.2 Inverter Specifications}

1. The inverter shall have a continuous power rating of $7200 \mathrm{VA}(30 \mathrm{~A})$ at $25^{\circ} \mathrm{C}$ $\left(77^{\circ} \mathrm{F}\right)$.

2. Nominal AC voltage shall be 240 VAC.

3. AC input voltage range shall be $\pm 10 \%$ of nominal.

4. Nominal AC frequency shall be $60 \mathrm{~Hz}$.

5. AC input frequency range shall be $\pm 5 \mathrm{~Hz}$.

6. Idle power consumption by the inverter shall be less than $60 \mathrm{~W}$.

7. Typical total harmonic distortion shall be less than or equal to $2 \%$, with a maximum no greater than $5 \%$.

8. Maximum output current of the inverter shall be no greater than 70 A $(233 \%$ of rated) for no longer than $1 \mathrm{~ms}$ and no greater than $50 \mathrm{~A}(140 \%)$ for no longer than $100 \mathrm{~ms}$.

9. AC overload capacity shall be less than 12,000 VA (167\%) for no longer than $100 \mathrm{~ms}$, less than 10,000 VA (139\%) for no longer than 5 seconds, and less than 8000 VA (111\%) for no longer than 30 minutes

10. Maximum AC input current shall be 60 A (200\%). 
11. The inverter shall accommodate one three conductor, four wire (L1, L2, N, GND) power port, rated at $30 \mathrm{~A}$ continuous.

12. The port on the inverter shall accommodate both power (L1, L2, N) and data communication between the meter and inverter via a single socket.

13. The inverter shall use the neutral connection $(\mathrm{N})$ provided by the meter.

14. An AC disconnect switch (L1 and L2) shall be included within the inverter.

15. Remote actuation of the disconnect switch shall be controllable from the meter, unless the disconnect switch has been manually locked.

16. The disconnect switch shall also be accessible for manual operation.

17. The open and closed status of the disconnect switch shall be visibly indicated on the inverter.

18. The disconnect switch shall be lockable open.

19. The disconnect switch shall not be lockable closed.

20. The inverter shall provide the meter with inverter status data (e.g. disconnect switch position, system enabled/disabled, etc.) through its communications link with the meter.

21. The inverter shall pass through the status data of the battery (e.g. state of charge, system enabled/disabled, etc.) to the meter through its communications link with the meter. 
22. The inverter shall have the ability to increment from zero to rated power in one second or less.

23. The inverter shall have the ability to decrement from zero to rated power in one second or less.

24. The firmware settings shall field accessible and adjustable.

25. The firmware settings shall remotely accessible and adjustable.

26. The inverter shall coordinate with the meter to perform a sync-check relay function for the purpose of synchronizing the ResBESS at the point of common connection.

27. The rated temperature range should be $0^{\circ} \mathrm{C}$ to $50^{\circ} \mathrm{C}$, with power derated beyond $25^{\circ} \mathrm{C}$.

28. The inverter efficiency should be greater than or equal to $93 \%$

29. The grid-interactive frequency range is not specified. IEEE 1547 defines a range of $59.3 \mathrm{~Hz}$ to $60.5 \mathrm{~Hz}$. The range shall be determined based on the desired frequency regulation capabilities of the unit.

30. The grid-interactive AC voltage range is not specified. IEEE 1547 defines a range of $\pm 10 \%$. The range shall be determined based on the desired voltage regulation capabilities of the unit.

31. The unit shall weight no greater than $50 \mathrm{lbs}(23 \mathrm{~kg})$. 
32. The DC input voltage range is not specified. This specification depends on the specifications of the battery.

33. Continuous DC charge and discharge currents are not specified. This specification depends on the specifications of the battery. 


\section{Appendix D: IEEE 1547}

The IEEE 1547 standard (Standard for Interconnecting Distributed Resources with Electric Power Systems) and its supplementary documents are referenced in this report, as they provide the prevailing standards in the power industry for DR implementation. The scope of IEEE 1547 "establishes criteria and requirements for interconnection of distributed resources with electric power systems". Its purpose is to provide "a uniform standard for interconnection of distributed resources with electric power systems." The subsections of the IEEE 1547 series are as follows:

- IEEE Std 1754, IEEE Standard for Distributed Resources Interconnected with Electric Power Systems.

- IEEE Std 1547.1, IEEE Standard for Conformance Test Procedures for Equipment Interconnecting Distributed Resources with Electric Power Systems.

- IEEE Std 1547.2, Application Guide for IEEE Std 1547 Standard for Interconnecting Distributed Resources with Electric Power Systems

- IEEE Std 1547.3, IEEE Guide for Monitoring, Information Exchange, and Control of Distributed Resources Interconnected with Electric Power Systems. 
- IEEE Std 1547.4, Guide for Design, Operation, and Integration of Distributed Resource Island Systems with Electric Power Systems.

- IEEE Std 1547.5, Technical Guidelines for Interconnection of Electric Power Sources Greater Than 10 MVA to the Power Transmission Grid.

- IEEE Std 1547.6, Recommended Practice for Interconnecting Distributed Resources with Electric Power Systems Distribution Secondary Networks

- IEEE Std 1547.7, Guide for Conducting Distribution Impact Studies for Distributed Resource Interconnection

- IEEE Std 1547.8, Update and expansion of IEEE Standard 1547 


\section{Appendix E: NERC CIP}

Here we examine NERC CIP-002, the NERC standard which governs identification of critical assets. The standard being enforced at the time this research was done was CIP-002-3, but the newer standard of CIP-002-5 became effective early in 2015. This study was undertaken in order to justify a claim that aggregated ResBESS must be a protected asset under NERC CIP rules. This section contains both an overview of the older and current standards, as well as their application to ResBESS units.

\section{E.1 CIP-002-3}

NERC Standard CIP-002-3, the older iteration, required "the identification and documentation of the Critical Cyber Assets [CCAs] associated with the Critical Assets that support the reliable operation of the Bulk Electric System [BES]". It directed entities to identify these assets through the application of a risk-based assessment. It was not clear in the standard what that risk assessment should be, though there were some guidelines on who should be doing the risk assessment, and which assets should be considered in that assessment.

The "Responsible Entity" in CIP-002-3 is someone is required to do the risk assessment and they are defined as: 
- Reliability Coordinator

- Balancing Authority

- Interchange Authority

- Transmission Service Provider

- Transmission Owner

- Transmission Operator
- Generator Owner

- Generator Operator

- Load Serving Entity

- NERC

- Regional Entity 
The assets required to be considered in the risk assessment are as follows:

1. Control centers and backup control centers performing the functions of the entities listed in the Applicability section of this standard.

2. Transmission substations that support the reliable operation of the Bulk Electric System.

3. Generation resources that support the reliable operation of the Bulk Electric System.

4. Systems and facilities critical to system restoration, including black start generators and substations in the electrical path of transmission lines used for initial system restoration.

5. Systems and facilities critical to automatic load shedding under a common control system capable of shedding $300 \mathrm{MW}$ or more.

6. Special Protection Systems that support the reliable operation of the Bulk Electric System.

7. Any additional assets that support the reliable operation of the Bulk Electric System that the Responsible Entity deems appropriate to include in its assessment.

Note that while aggregated storage is not explicitly called out in this list, items 3,4,5 and 7 could leave the door open for its inclusion in the risk assessment - though the entity would need to make the case of why it 'deems appropriate' their inclusion, and this may still not guarantee their classification as CCAs. [25] 
Thus, under the colder rule CIP-002-3, it is possible that a system of ResBESS units could be classified as a CCA, and need to be protected; but there is not a direct mandate for this decision.

\section{E.2 CIP-002-5}

NERC Standard CIP-002-5, the newest iteration which went into effect in early 2016, is intended "...to identify and categorize BES Cyber Systems and their associated BES Cyber Assets for the application of cyber security requirements commensurate with the adverse impact that loss, compromise, or misuse of those BES Cyber

Systems could have on the reliable operation of the BES". There is much more detail in this version than within the prior: the lengths of the documents are 5 pages and 35 pages respectively.

One area with more information is how to go about identifying assets. Rather than the entity-driven risk-based assessment in version 3, the new standard provides 'bright-line' criteria for categorization of BES Cyber Systems based on "the impact of their associated Facilities, systems, and equipment, which if destroyed, degraded, misused, or otherwise rendered unavailable, would affect the reliable operation of the Bulk Electric System". In other words, one is no longer required to decide whether an asset is a cyber-risk, as it is spelled out for explicitly. 


\section{E.2.0.1 Asset Identification per CIP-002-5}

BES Cyber Assets and Cyber Systems - this is a new distinction in version 5, and is merely NERC using the NIST ${ }^{24}$ Risk Management Framework, and their use of an analogous term "information system" as the target for categorizing and applying security controls, as a way of defining groups of Cyber Assets as Cyber Systems. The Responsible Entity decides the granularity (Do 30 assets count as a system? Do we include an entire control system, or just part of one?), but the gist is that protection can now be applied to groupings rather than individual assets as long as the protection of the group protects the individuals. The flip side is if a location or asset is very critical, then the group becomes critical also; this will be covered in more detail in later sections.

Back to the 'bright-line' criteria. The methods of critical infrastructure identification are now simply an examination of how or whether an asset affects the reliable operation of the BES, and/or whether an asset (or the lack thereof) can affect the real-time operations of the BES.

For the former, affecting reliability, the measurement is a determination of whether the BES Cyber System performs or supports any BES reliability function as defined in its relationships with other functional entities in the NERC Functional Model. In essence, 'do you use a system or set of assets to meet our (NERC's) reliability standards? If so, it can affect reliability; protect it.'

\footnotetext{
${ }^{24}$ National Institute of Standards and Technology
} 
In the case of real-time operations, it's an even easier diagnosis. NERC defines BES Cyber Assets as those Cyber Assets that, if rendered unavailable, degraded, or misused, would adversely impact the reliable operation of the BES within $15 \mathrm{~min}$ utes of the activation or exercise of the compromise. Furthermore, this time window is not allowed to include in its consideration the activation of redundant BES Cyber Assets or BES Cyber Systems: from the cyber security standpoint, redundancy does not mitigate cyber security vulnerabilities [26].

All of this identification is done with the goal of deciding whether to place the assets in NERC's new 'BES Cyber Asset' impact categories.

\section{E.2.0.2 BES Impact Categories}

The BES impact categories NERC has created in rev. 5 are High Impact, Medium Impact and Low Impact, and depending on how the piece of infrastructure is identified (or how the BES Facility it's located within or associated with is identified), it can be listed as a low/medium/high impact BES Cyber Asset. This categorization matters because each category has different levels of protection required under the remaining CIP standards. These new impact facilities do map fairly well to pre-identified CCAs; an example of how old CCAs fit into revision 5 can be seen in Figure E.1. 


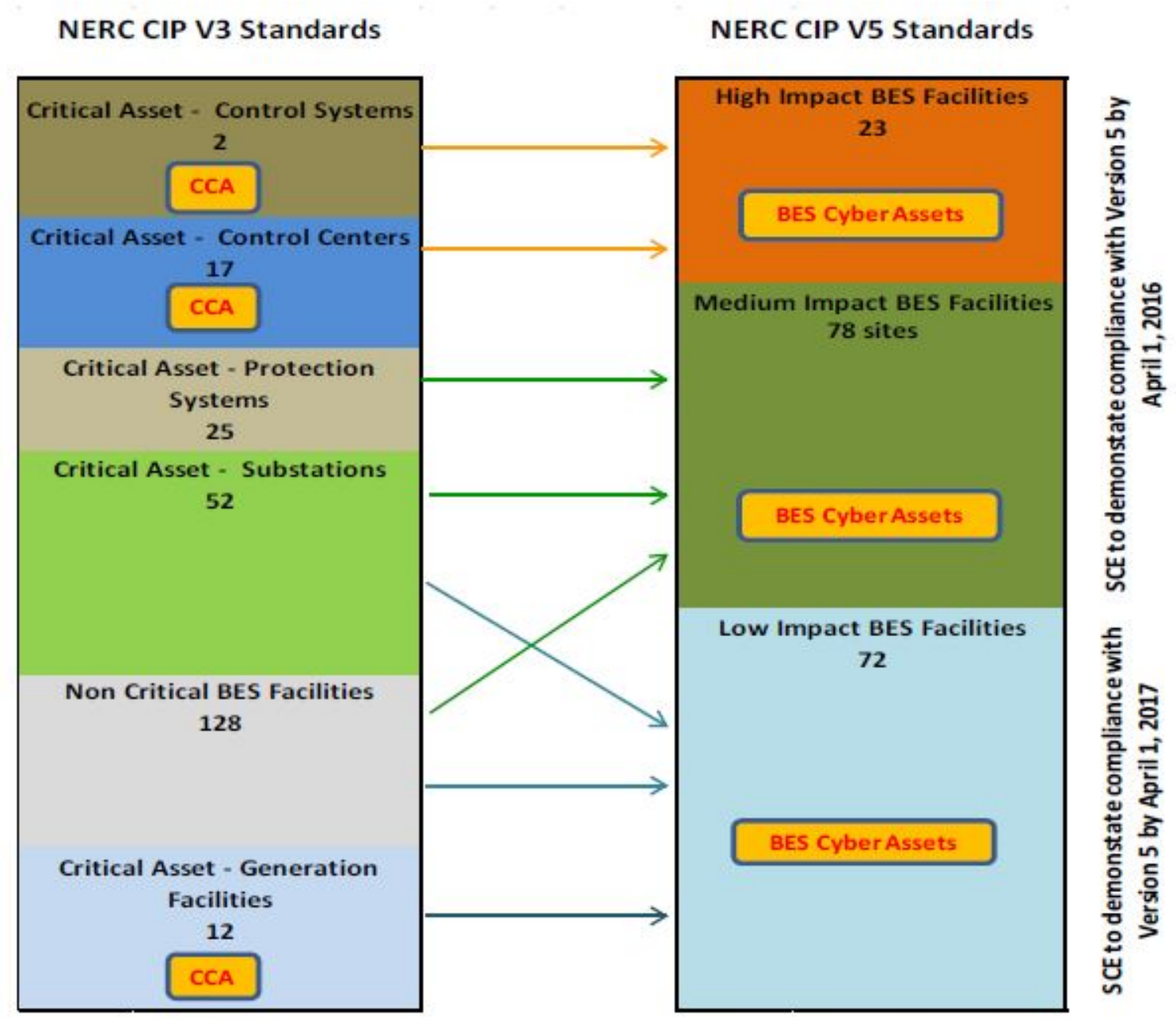

Figure E.1: BES Cyber Asset vs Critical Cyber Assets [27]

As observed in the previous figure, certain types of assets and locations from version 3 become BES Facilities with varying impacts in version 5. This is spelled out in the standard; but the most important thing is not which facilities get which impact rating, but this particular line: "Each BES Cyber System used by and located at any of the following...", whereafter the standard continues with the classification of locations: control centers used to perform the obligations of the Reliability Coordinator, or of the Balancing Authority, or of a Transmission Operator for one or 
more of the assets that meet certain criteria, or of the Generator Operator for one or more of the assets...there's a pattern, and it is that facilities at high-voltage locations or with control capabilities which can affect high-voltage locations, automatically make assets located there or used by them a part of that facility.

\section{E.2.1 ResBESS and CIP-002}

In the context of the previous material, we feel confident in identifying aggregated ResBESS and their data as part of a Medium Impact BES Facility. They would be intended for use in system reliability in ways that, were they taken out of the BES for more than 15 minutes - and remember, no redundant systems are allowed to be considered here - would result in some level of adverse impact on the BES.

We would recommend, however, that an entity studying this topic consider classifying them as part of a High-Impact BES Facility. To be considered in this category, they would need to have their data used by control centers, and be constantly tied to real-time operations and reliability - both things that the ResBESS research indicates are likely to be intended uses. A forward thinking organization could make the categorization now, and be prepared for CIP-002-6 where aggregated storage may be specifically called out as a new type of asset which needs to be considered as high-impact. 
Appendix F: Additional Device Drawings and System Schematics

F.1 Meter-Base Adapter

(This space left blank intentionally)

\section{F.2 Field Prototype}




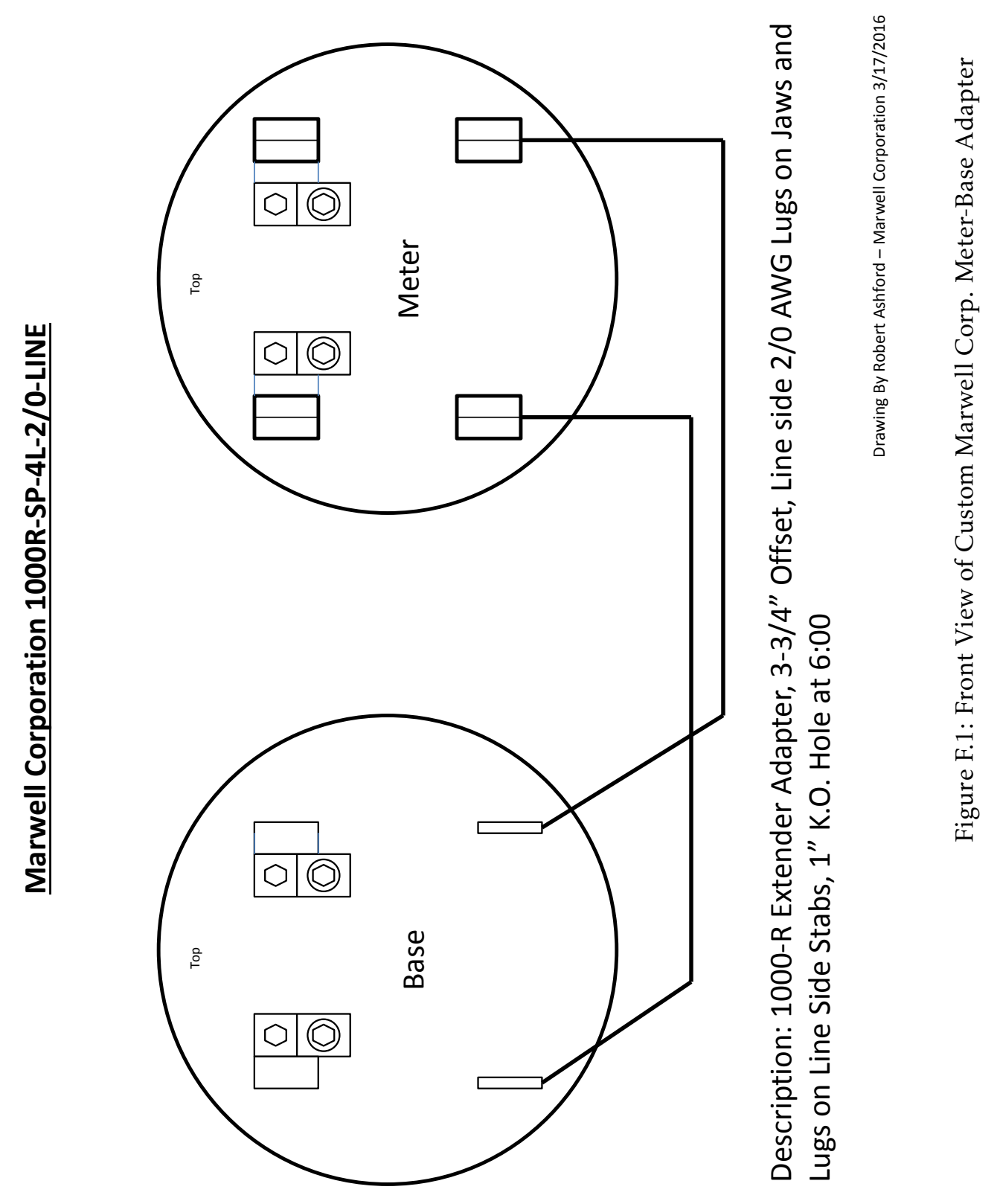



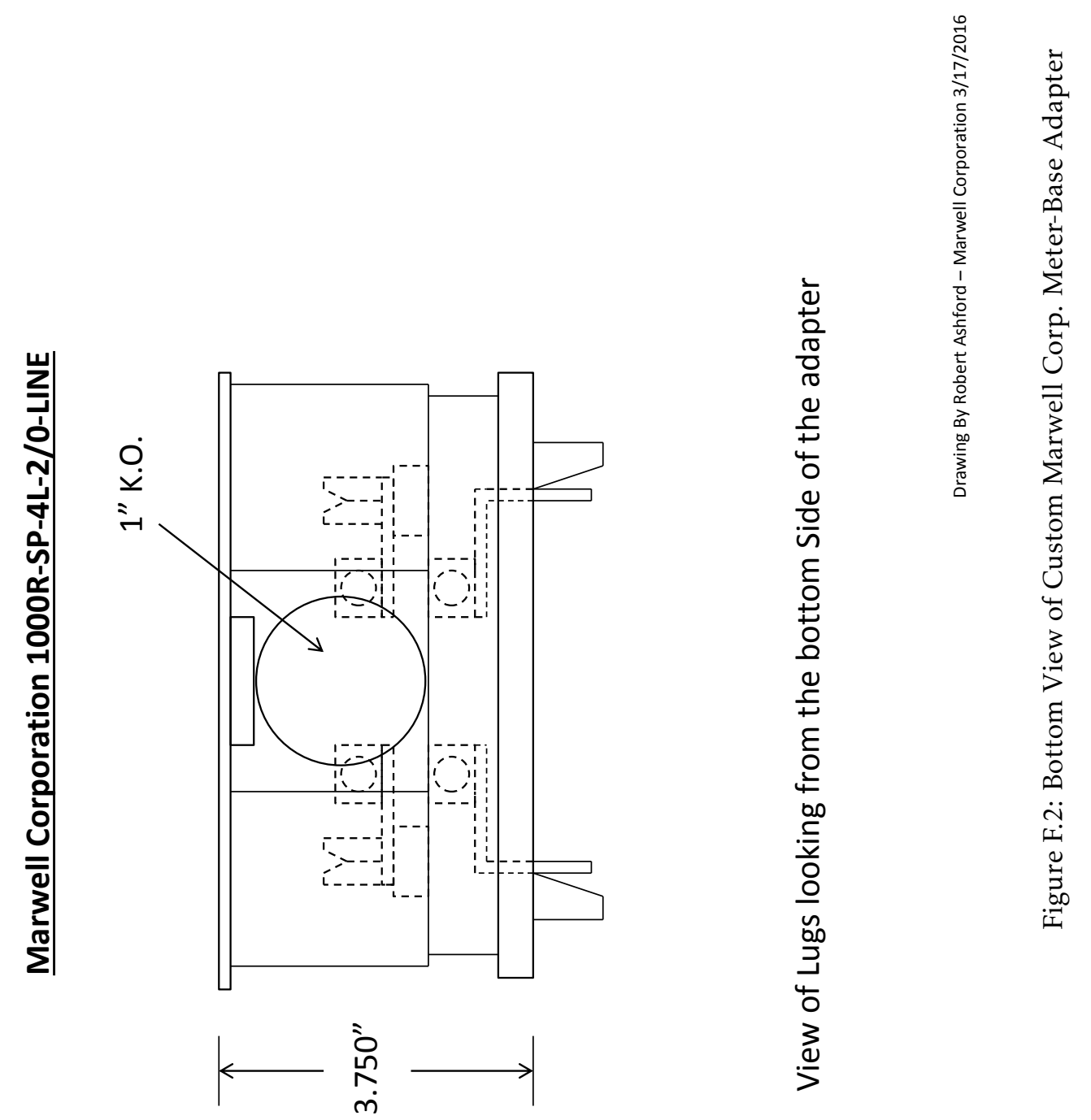


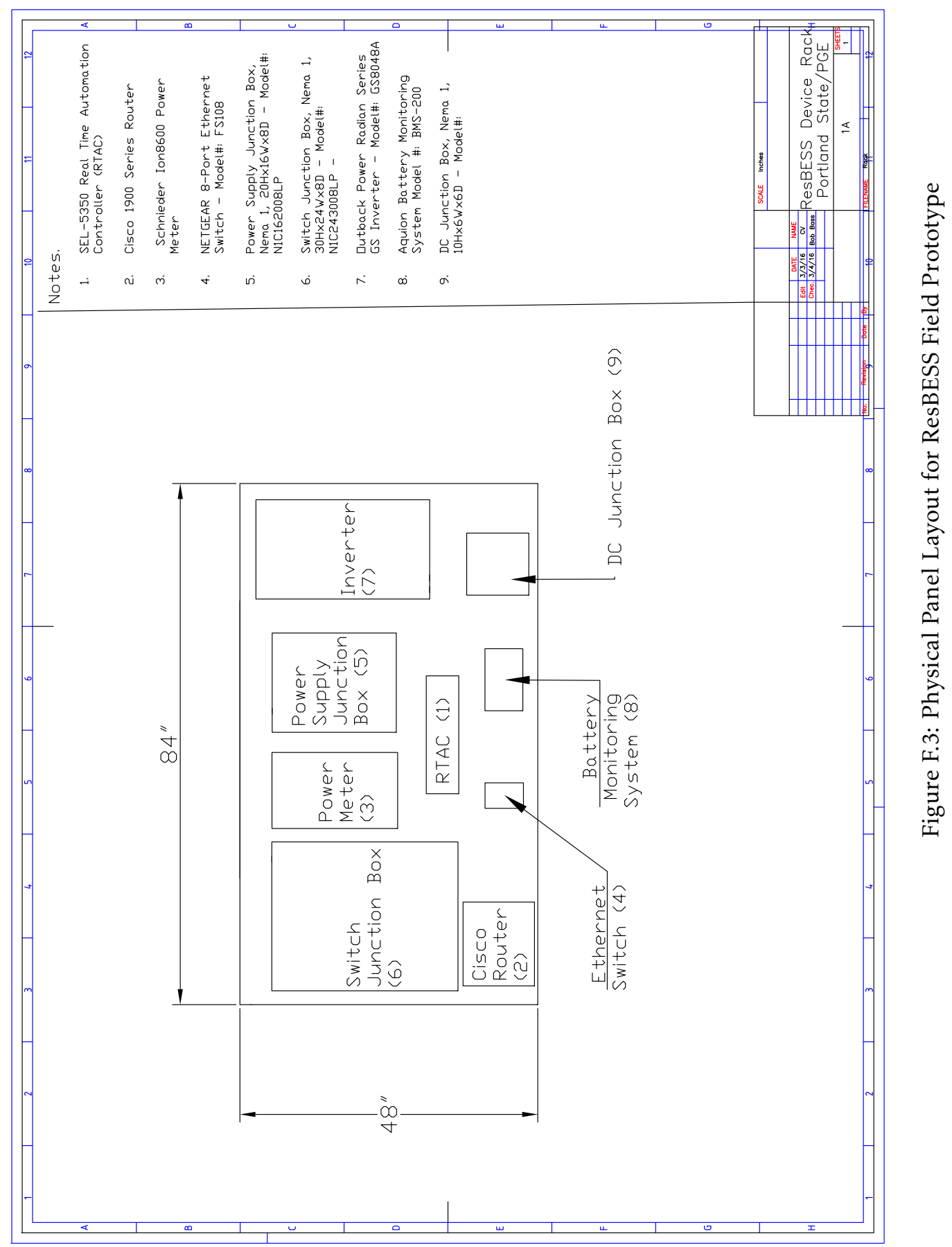




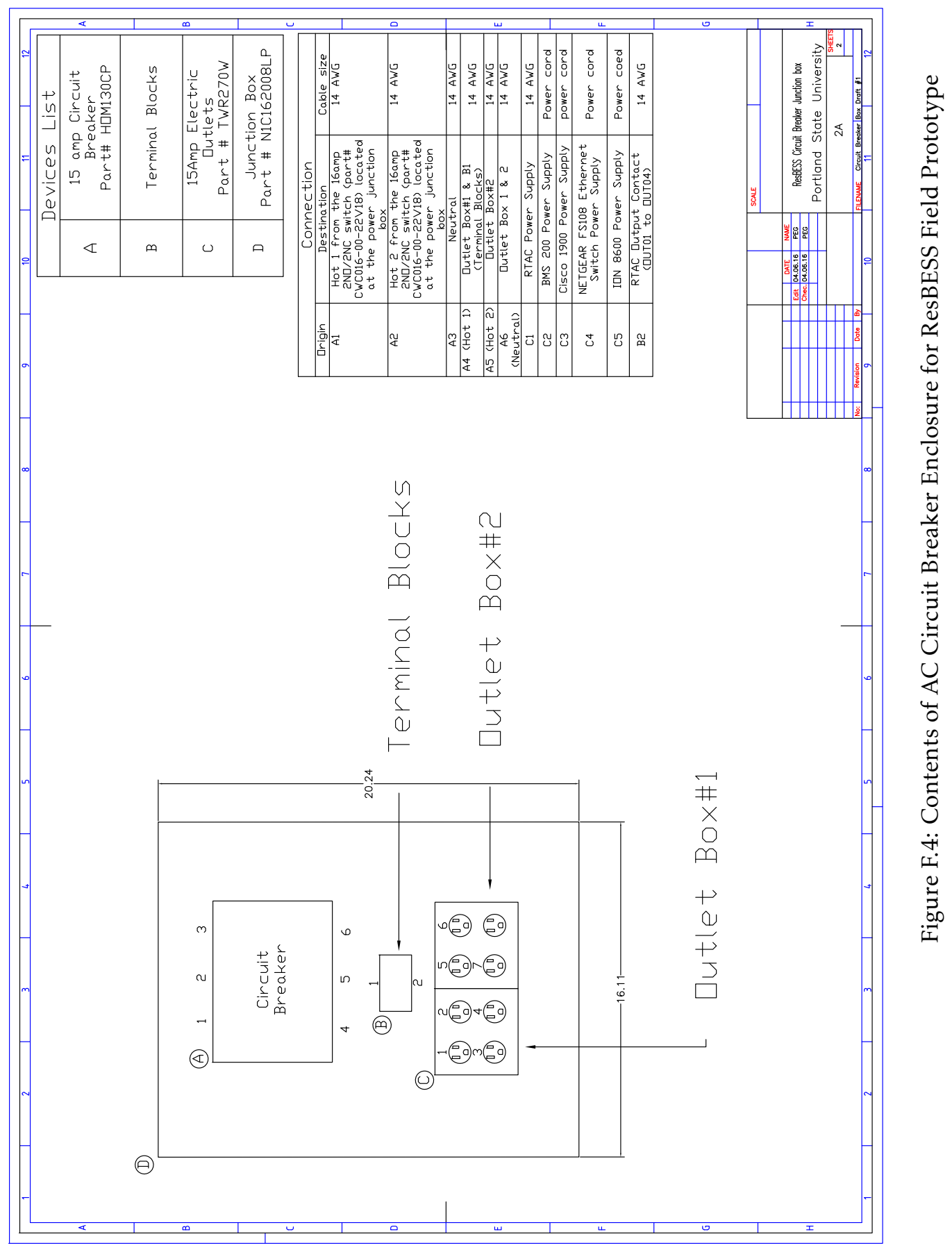




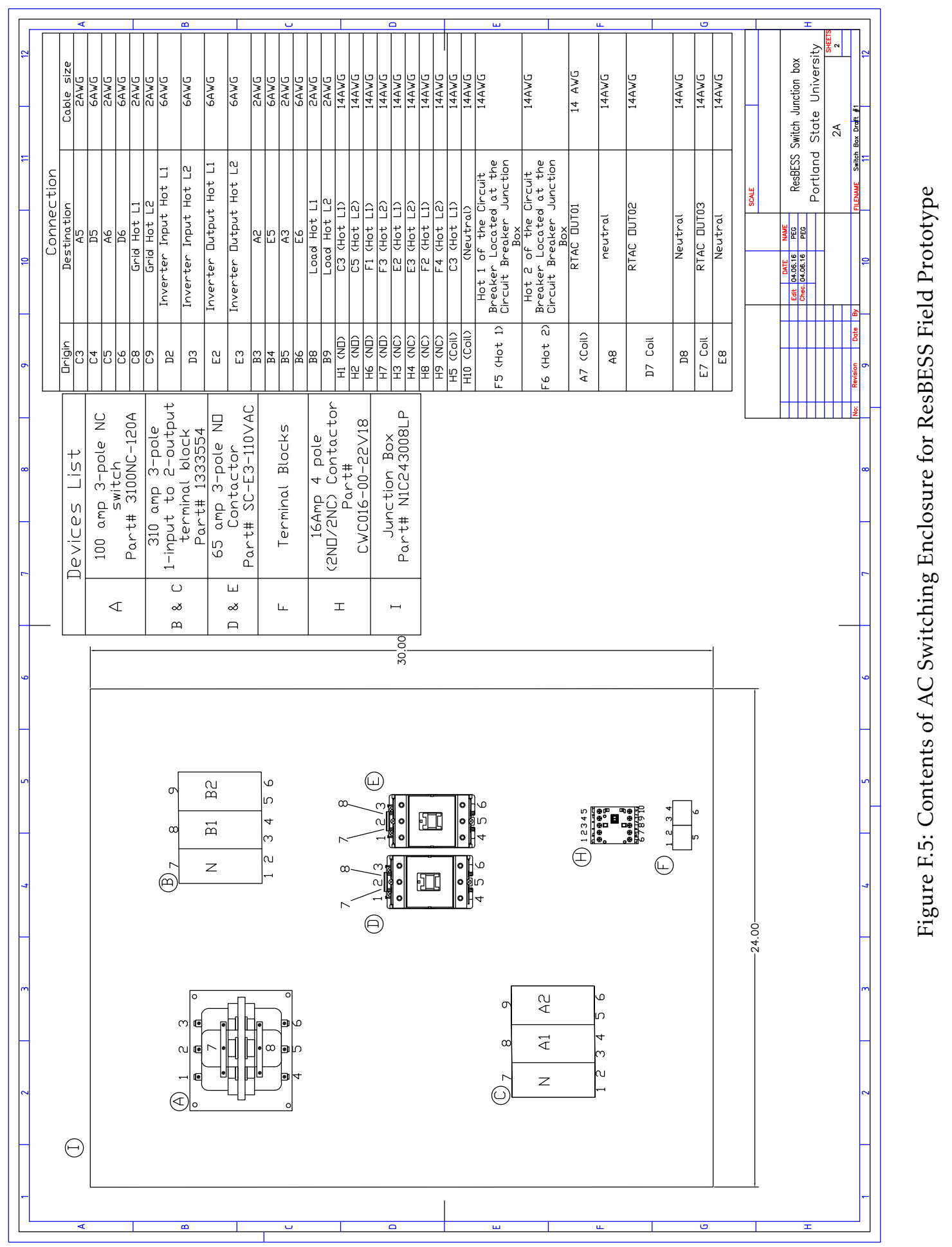




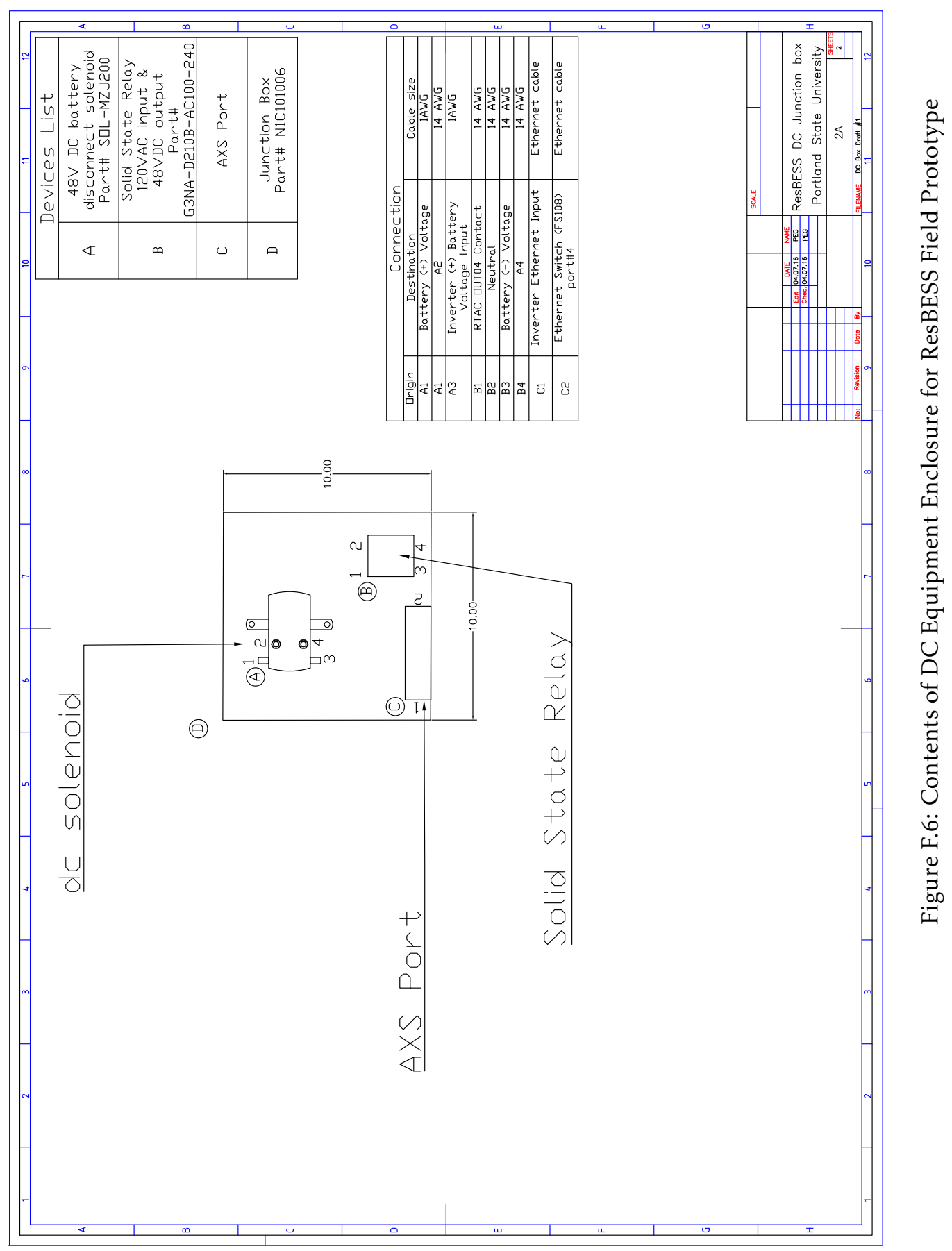


Appendix G: Switching Logic

This Appendix details the state transitions that will occur as Status Signals change. Numbers in parenthesis (e.g. Grid Tied State (111)) represent the disconnect switch positions for the utility, residence and inverter switches, respectively. Refer to Table 4.1 for a summary of the Status Signals, Switch Positions and System States.

\section{G.1 Grid Tied State Transitions}

Grid Tied State (111):

('Inverter 0perable' AND 'Battery Operable') TRUE 'Nominal Service' TRUE

('Grid Steady-state' AND 'Grid Online') TRUE

\section{Transition to Maintenance:}

IF ('Inverter Operable' AND 'Battery Operable') becomes FALSE

AND 'Nominal Service' remains TRUE

AND ('Grid Steady-State' AND 'Grid Online') remains TRUE

THEN switch to Maintenance (110) 


\section{Transition to Backup:}

IF ('Inverter Operable' AND 'Battery Operable') remains TRUE AND 'Nominal Service' remains TRUE

AND ('Grid Steady-State' OR 'Grid Online') becomes FALSE

THEN switch to Backup (011)

\section{Transition to Service Disconnect:}

IF ('Inverter Operable' AND 'Battery Operable') remains TRUE AND 'Nominal Service' becomes FALSE

AND ('Grid Steady-State' AND 'Grid Online') remains TRUE THEN switch to Service Disconnect (101)

\section{Transition to No Service:}

IF ('Inverter Operable' AND 'Battery Operable') becomes FALSE

AND 'Nominal Service' becomes FALSE

AND ('Grid Steady-State' OR 'Grid Online') becomes FALSE

ELSE IF

('Inverter Operable' AND 'Battery Operable') becomes FALSE

AND 'Nominal Service' becomes FALSE

AND ('Grid Steady-State' OR 'Grid Online') remains TRUE 
ELSE IF

('Inverter Operable' AND 'Battery Operable') becomes FALSE

AND 'Nominal Service' remains TRUE

AND ('Grid Steady-State' OR 'Grid Online') becomes FALSE

ELSE IF

('Inverter Operable' AND 'Battery Operable') remains TRUE

AND 'Nominal Service' becomes FALSE

AND ('Grid Steady-State' OR 'Grid Online') becomes FALSE

THEN switch to No Service (000) 


\section{G.2 Maintenance State Transitions}

Maintenance State (110):

('Inverter Operable' AND 'Battery Operable') FALSE

'Nominal Service' TRUE

( 'Grid Steady-state' AND 'Grid Online') TRUE

\section{Transition to Grid Tied:}

IF ('Inverter Operable' AND 'Battery Operable') becomes TRUE

AND 'Nominal Service' remains TRUE

AND ('Grid Steady-State' AND 'Grid Online') remains TRUE

THEN switch to Grid Tied (111)

\section{Transition to Backup:}

IF ('Inverter Operable' AND 'Battery Operable') becomes TRUE AND 'Nominal Service' remains TRUE

AND ('Grid Steady-State' OR 'Grid Online') becomes FALSE

THEN switch to Backup (011)

Transition to Service Disconnect: 


\author{
IF ('Inverter Operable' AND 'Battery Operable') becomes TRUE \\ AND 'Nominal Service' becomes FALSE \\ AND ('Grid Steady-State' OR 'Grid Online') remains TRUE \\ THEN switch to Service Disconnect (101)
}




\section{Transition to No Service:}

IF ('Inverter Operable' AND 'Battery Operable') remains FALSE

AND 'Nominal Service' becomes FALSE

AND ('Grid Steady-State' OR 'Grid Online') becomes FALSE

ELSE IF

('Inverter Operable' AND 'Battery Operable') remains FALSE

AND 'Nominal Service' becomes FALSE

AND ('Grid Steady-State' OR 'Grid Online') remains TRUE

ELSE IF

('Inverter Operable' AND 'Battery Operable') becomes TRUE

AND 'Nominal Service' remains TRUE

AND ('Grid Steady-State' OR 'Grid Online') remains TRUE

ELSE IF

('Inverter Operable' AND 'Battery Operable') becomes TRUE

AND 'Nominal Service' becomes FALSE

AND ('Grid Steady-State' OR 'Grid Online') becomes FALSE

THEN switch to No Service (000) 


\section{G.3 Backup State Transitions}

Backup State (011):

('Inverter 0perable' AND 'Battery Operable') TRUE

'Nominal Service' TRUE

('Grid Steady-state' AND 'Grid Online') FALSE

\section{Transition to Grid Tied:}

IF ('Inverter Operable' AND 'Battery Operable') remains TRUE

AND 'Nominal Service' remains TRUE

AND ('Grid Steady-State' OR 'Grid Online') becomes TRUE

THEN switch to Grid Tied (111)

\section{Transition to Maintenance:}

IF ('Inverter Operable' AND 'Battery Operable') becomes FALSE

AND 'Nominal Service' remains TRUE

AND ('Grid Steady-State' AND 'Grid Online') becomes TRUE

THEN switch to Maintenance (110)

Transition to Service Disconnect: 


\author{
IF ('Inverter Operable' AND 'Battery Operable') remains TRUE \\ AND 'Nominal Service' becomes FALSE \\ AND ('Grid Steady-State' OR 'Grid Online') becomes TRUE \\ THEN switch to Service Disconnect (101)
}




\section{Transition to No Service:}

IF ('Inverter Operable' AND 'Battery Operable') becomes FALSE

AND 'Nominal Service' becomes FALSE

AND ('Grid Steady-State' OR 'Grid Online') remains FALSE

ELSE IF ('Inverter Operable' AND 'Battery Operable') becomes FALSE

AND 'Nominal Service' becomes FALSE

AND ('Grid Steady-State' OR 'Grid Online') becomes TRUE

ELSE IF ('Inverter Operable' AND 'Battery Operable') becomes FALSE

AND 'Nominal Service' remains TRUE

AND ('Grid Steady-State' OR 'Grid Online') remains FALSE

ELSE IF ('Inverter Operable' AND 'Battery Operable') remains TRUE

AND 'Nominal Service' becomes FALSE

AND ('Grid Steady-State' OR 'Grid Online') remains FALSE

THEN switch to No Service (000) 


\section{G.4 Service Disconnect State Transitions}

Service Disconnect State (101):

('Inverter 0perable' AND 'Battery Operable') TRUE

'Nominal Service' FALSE

('Grid Steady-state' AND 'Grid Online') TRUE

\section{Transition to Grid Tied:}

IF ('Inverter Operable' AND 'Battery Operable') remains TRUE

AND 'Nominal Service' becomes TRUE

AND ('Grid Steady-State' OR 'Grid Online') remains TRUE

THEN switch to Grid Tied (111)

\section{Transition to Maintenance:}

IF ('Inverter Operable' AND 'Battery Operable') becomes FALSE

AND 'Nominal Service' becomes TRUE

AND ('Grid Steady-State' AND 'Grid Online') remains TRUE

THEN switch to Maintenance (110)

Transition to Backup: 


\author{
IF ('Inverter Operable' AND 'Battery Operable') remains TRUE \\ AND 'Nominal Service' becomes TRUE \\ AND ('Grid Steady-State' OR 'Grid Online') becomes FALSE \\ THEN switch to Backup (011)
}




\section{Transition to No Service:}

IF ('Inverter Operable' AND 'Battery Operable') becomes FALSE

AND 'Nominal Service' remains FALSE

AND ('Grid Steady-State' OR 'Grid Online') becomes FALSE

ELSE IF

('Inverter Operable' AND 'Battery Operable') becomes FALSE

AND 'Nominal Service' remains FALSE

AND ('Grid Steady-State' OR 'Grid Online') remains TRUE

ELSE IF

('Inverter Operable' AND 'Battery Operable') becomes FALSE

AND 'Nominal Service' becomes TRUE

AND ('Grid Steady-State' OR 'Grid Online') becomes FALSE

ELSE IF

('Inverter Operable' AND 'Battery Operable') remains TRUE

AND 'Nominal Service' remains FALSE

AND ('Grid Steady-State' OR 'Grid Online') becomes FALSE

THEN switch to No Service (000) 


\section{G.5 No Service State Transitions}

No Service States (000):

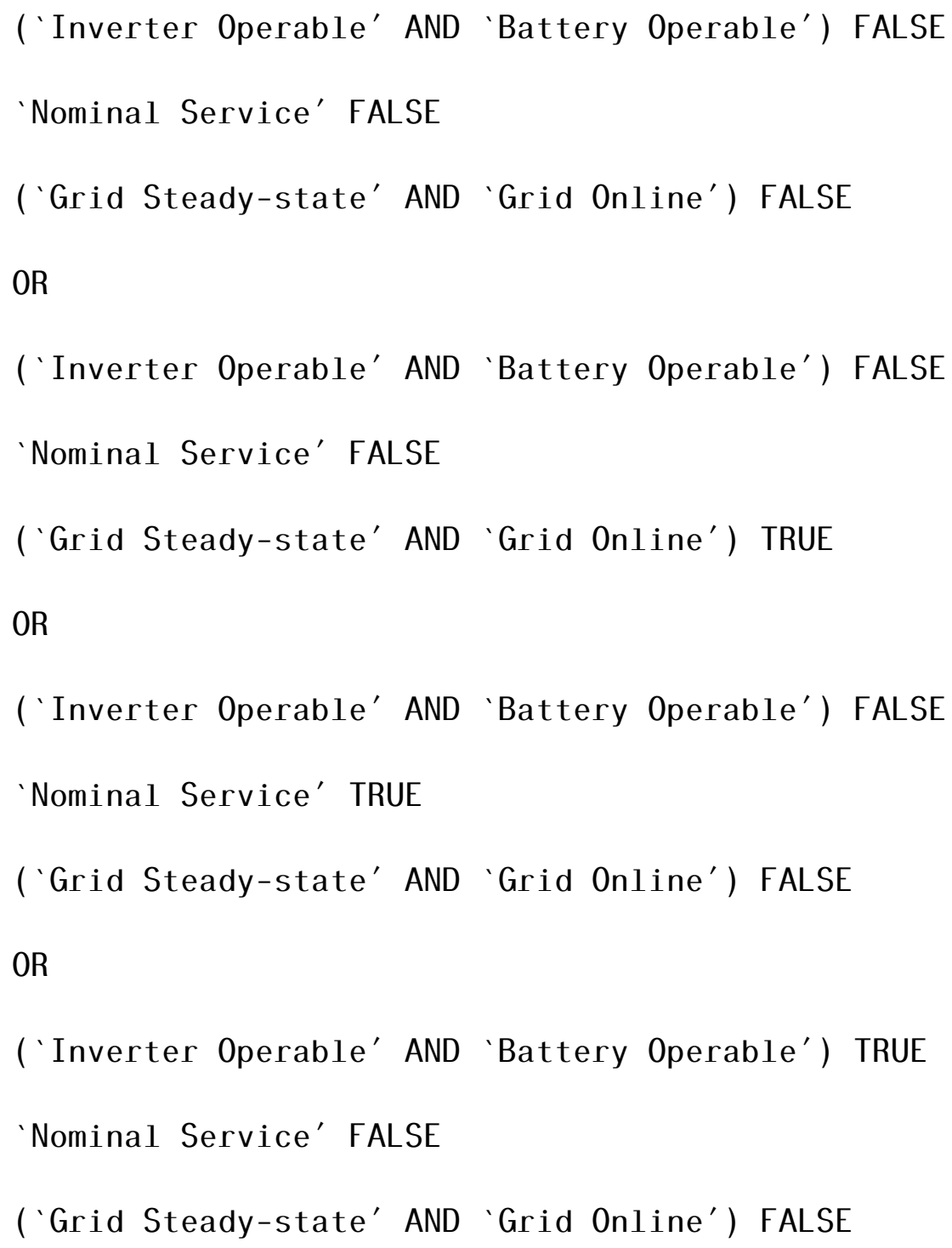

Transition to Grid Tied:

From Status Signal 000: 
IF ('Inverter Operable' AND 'Battery Operable') becomes TRUE AND 'Nominal Service' becomes TRUE

AND ('Grid Steady-State' OR 'Grid Online') becomes TRUE THEN switch to Grid Tied (111)

From Status Signal 001:

IF ('Inverter Operable' AND 'Battery Operable') becomes TRUE AND 'Nominal Service' becomes TRUE AND ('Grid Steady-State' OR 'Grid Online') remains TRUE THEN switch to Grid Tied (111)

From Status Signal 010:

IF ('Inverter Operable' AND 'Battery Operable') becomes TRUE AND 'Nominal Service' remains TRUE AND ('Grid Steady-State' OR 'Grid Online') becomes TRUE THEN switch to Grid Tied (111)

From Status Signal 100:

IF ('Inverter Operable' AND 'Battery Operable') remains TRUE AND 'Nominal Service' becomes TRUE AND ('Grid Steady-State' OR 'Grid Online') becomes TRUE 
THEN switch to Grid Tied (111)

\section{Transition to Maintenance:}

From Status Signal 000:

IF ('Inverter Operable' AND 'Battery Operable') remains FALSE AND 'Nominal Service' becomes TRUE

AND ('Grid Steady-State' OR 'Grid Online') becomes TRUE

THEN switch to Maintenance (110)

From Status Signal 001:

IF ('Inverter 0perable' AND 'Battery Operable') remains FALSE AND 'Nominal Service' becomes TRUE

AND ('Grid Steady-State' OR 'Grid Online') remains TRUE THEN switch to Maintenance (110)

From Status Signal 010:

IF ('Inverter Operable' AND 'Battery Operable') remains FALSE AND 'Nominal Service' remains TRUE

AND ('Grid Steady-State' OR 'Grid Online') becomes TRUE THEN switch to Maintenance (110) 
From Status Signal 100:

IF ('Inverter 0perable' AND 'Battery Operable') becomes FALSE

AND 'Nominal Service' becomes TRUE

AND ('Grid Steady-State' OR 'Grid Online') becomes TRUE

THEN switch to Maintenance (110)

\section{Transition to Backup:}

From Status Signal 000:

IF ('Inverter Operable' AND 'Battery Operable') becomes TRUE AND 'Nominal Service' becomes TRUE

AND ('Grid Steady-State' OR 'Grid Online') remains FALSE

THEN switch to Backup (011)

From Status Signal 001:

IF ('Inverter Operable' AND 'Battery Operable') becomes TRUE

AND 'Nominal Service' becomes TRUE

AND ('Grid Steady-State' OR 'Grid Online') becomes FALSE

THEN switch to Backup (011)

From Status Signal 010:

IF ('Inverter Operable' AND 'Battery Operable') becomes TRUE 


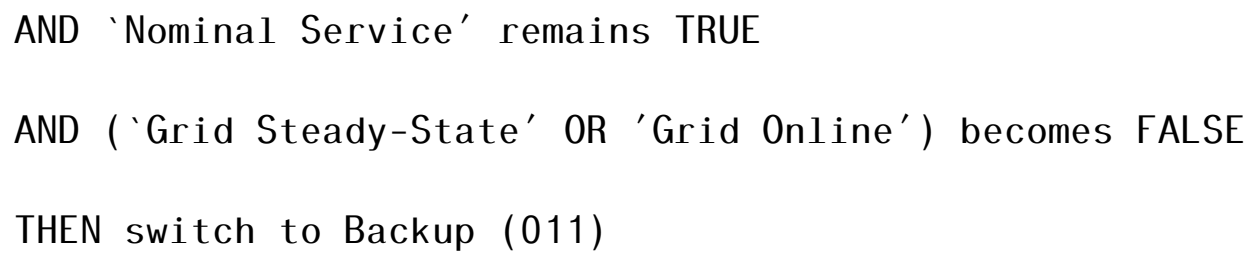




\section{Appendix H: RTAC Logic}

Discharge and Charge are booleans from PGE indicating desired behavior of prototype.

IF (Discharge AND (NOT Charge)) = TRUE THEN

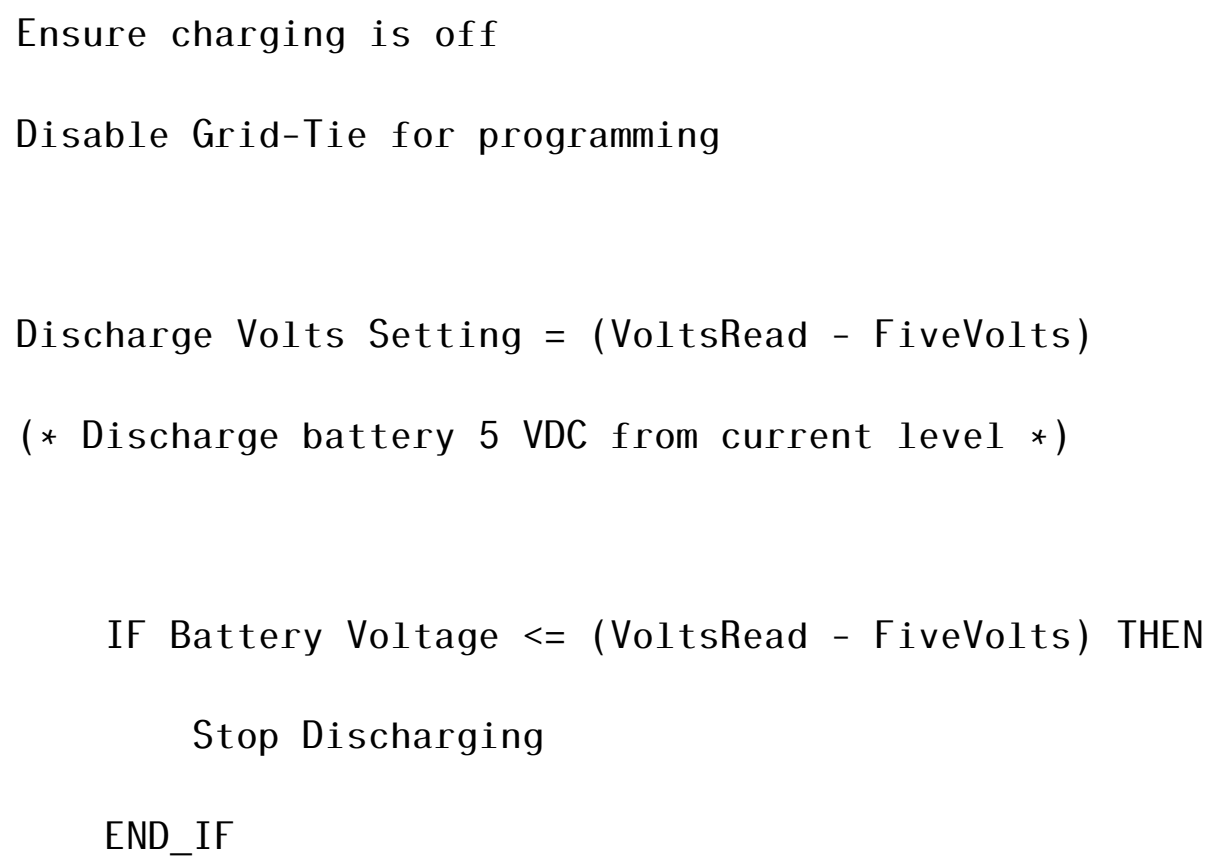

Charge for 0 hours, i.e. do not charge

Float FOR 0 hours, i.e. DO NOT float-charge 
Grid-Tie enabled so Discharge will begin

ELSE IF (Charge AND (NOT Discharge)) = TRUE THEN

Ensure Charging off for programming

Bulk Charge disabled

Set to Charge at 57.6 VDC

(* Manufacturer Recommended Charge Voltage *)

Charge for 10 hours

Float for 4 hours

Charging on

Bulk Charge enabled

Grid Tie Mode Enabled

IF Current $<0$ THEN

( $*$ Checks to see if charging has stopped $*$ )

(* or if current has reversed $*$ )

Reset charge timers to 0

Stop charging 


\section{END_IF}

ELSE

Go into standby:

grid-tie is disabled

\section{END_IF}




\section{Appendix I: Equations}

\section{I.1 SAIDI}

System Average Interruption Duration Index (SAIDI):

$$
S A I D I=\frac{\sum U_{i} N_{i}}{N_{T}}
$$

Where $U_{i}$ is the annual outage time for location $i, N_{i}$ is the number of customers for location $i$ and $N_{T}$ is the total number of customers served. Thus, SAIDI is measured in units of time, and is usually calculated for the course of a year. In other words,

$$
\text { SAIDI }=\frac{\text { sum of all customer interruption durations }}{\text { total number of customers served }}
$$

\section{I.2 SAIFI}

System Average Interruption Frequency Index (SAIFI):

$$
S A I F I=\frac{\sum \lambda_{i} N_{i}}{N_{T}}
$$

Where $\lambda_{i}$ is the failure rate for location $i, N_{i}$ is the number of customers for location $i$ and $N_{T}$ is the total number of customers served. Thus, SAIFI is measured in units of interruptions per customer, and is usually calculated for the course of a year. In other words,

$$
\text { SAIFI }=\frac{\text { total number of customer interruptions }}{\text { total number of customers served }}
$$




\section{I.3 MAIFI}

Momentary Average Interruption Frequency Index (MAIFI):

$$
M A I F I=\frac{\sum I D_{i} N_{i}}{N_{T}}
$$

Where $I D_{i}$ is the number of interrupting device operations at location $i, N_{i}$ is the number of customers momentarily interrupted for location $i$ and $N_{T}$ is the total number of customers served. Thus, MAIFI is measured in units of momentary interruptions ${ }^{25}$ per customer, and is usually calculated for the course of a year. In other words,

$$
\text { MAIFI }=\frac{\text { total number of customer interruptions less than defined time }}{\text { total number of customers served }}
$$

\footnotetext{
${ }^{25}$ PGE uses 5 minutes or less to define "momentary."
} 